\title{
Flips of moduli spaces and transition formulas for Donaldson polynomial invariants of rational surfaces
}

\author{
Robert Friedman AND Zhenbo QIN ${ }^{1}$
}

\section{Introduction.}

In [7], Donaldson has defined polynomial invariants for smooth simply connected 4-manifolds with $b_{2}^{+} \geq 3$. These invariants have also been defined for 4-manifolds with $b_{2}^{+}=1$ in $[25,17,18]$, along lines suggested by the work of Donaldson in [5]. In this case, however, they depend on an additional piece of information, namely a chamber defined on the positive cone of $H^{2}(X ; \mathbb{R})$ by a certain locally finite set of walls. Explicitly, let $X$ be a simply connected, oriented, and closed smooth 4-manifold with $b_{2}^{+}=1$ where $b_{2}^{+}$is the number of positive eigenvalues of the quadratic form $q_{X}$ when diagonalized over $\mathbb{R}$. Let

$$
\Omega_{X}=\left\{x \in H^{2}(X, \mathbb{R}) \mid x^{2}>0\right\}
$$

be the positive cone. Fix a class $\Delta$ in $H^{2}(X, \mathbb{Z})$ and an integer $c$ such that $d=4 c-\Delta^{2}-3$ is nonnegative. A wall of type $(\Delta, c)$ is a nonempty hyperplane:

$$
W^{\zeta}=\left\{x \in \Omega_{X} \mid x \cdot \zeta=0\right\}
$$

in $\Omega_{X}$ for some class $\zeta \in H^{2}(X, \mathbb{Z})$ with $\zeta \equiv \Delta(\bmod 2)$ and $\Delta^{2}-4 c \leq$ $\zeta^{2}<0$. The connected components of the complement in $\Omega_{X}$ of the walls of type $(\Delta, c)$ are the chambers of type $(\Delta, c)$. Then the Donaldson polynomial invariants of $X$ associated to $\Delta$ and $c$ are defined with respect to chambers of type $(\Delta, c)$. The invariants only depend on the class $w=\Delta \bmod 2 \epsilon$ $H^{2}(X ; \mathbb{Z} / 2 \mathbb{Z})$ and the integer $p=\Delta^{2}-4 c=-d-3$, and we shall often refer to walls and chambers of type $(w, p)$ as well. We shall write $D_{w, p}^{X}(\mathcal{C})$ for the Donaldson polynomial corresponding to the $S O(3)$ bundle $P$ with invariants $w_{2}(P)=w$ and $p_{1}(P)=p$, depending on the chamber $\mathcal{C}$.

${ }^{1}$ The first author was partially supported by NSF grants DMS-90-06116 and DMS-92-03940. The second author was partially supported by a grant from the ORAU Junior Faculty Enhancement Award Program and by NSF grants DMS-9100383 and DMS-94-00729. 
A basic question is then the following: Suppose that $\mathcal{C}_{+}$and $\mathcal{C}_{-}$are separated by a single wall $W^{\zeta}$. Here there may be more than one class $\zeta$ of type $(\Delta, c)$ defining $W^{\zeta}$. Then find a formula for the difference

$$
\delta_{w, p}^{X}\left(\mathcal{C}_{+}, \mathcal{C}_{-}\right)=D_{w, p}^{X}\left(\mathcal{C}_{+}\right)-D_{w, p}^{X}\left(\mathcal{C}_{-}\right) .
$$

We shall refer to such a difference as a transition formula.

There has been considerable interest in the above problem. The first result in this direction is due to Donaldson in [5], who gave a formula in case $\Delta=0$ and $c=1$. Kotschick [17] showed that, on the part of the symmetric algebra generated by 2 -dimensional classes, $\delta_{w, p}^{X}\left(\mathcal{C}_{+}, \mathcal{C}_{-}\right)= \pm \zeta^{d}$ for $\zeta^{2}=-\left(4 c-\Delta^{2}\right)=p$, and that $\delta_{w, p}^{X}\left(\mathcal{C}_{+}, \mathcal{C}_{-}\right)$is in fact always divisible by $\zeta$, except when $p=-5$ and $\zeta^{2}=-1$ (cf. also Mong [25] for some partial results along these lines). For a rational ruled surface $X$, all the transition formulas for $\Delta=0$ and $2 \leq c \leq 4$ have been determined in [25, 35, 22]. Using a gauge-theoretic approach, Yang [37] settled the problem for $\Delta=0$ and $c=2$, and computed the degree 5 Donaldson polynomials for rational surfaces. The known examples and the work of Kotschick and Morgan [18] raise the following rather natural conjecture:

Conjecture. The transition formula $\delta_{w, p}^{X}\left(\mathcal{C}_{+}, \mathcal{C}_{-}\right)$is a homotopy invariant of the pair $(X, \zeta)$; more precisely, if $\phi$ is an oriented homotopy equivalence from $X^{\prime}$ to $X$, then

$$
\delta_{\phi^{*} w, p}^{X^{\prime}}\left(\phi^{*}\left(\mathcal{C}_{+}\right), \phi^{*}\left(\mathcal{C}_{-}\right)\right)=\phi^{*} \delta_{w, p}^{X}\left(\mathcal{C}_{+}, \mathcal{C}_{-}\right) .
$$

We remark that this conjecture is essentially equivalent to the following statement: the transition formula $\delta_{w, p}^{X}\left(\mathcal{C}_{+}, \mathcal{C}_{-}\right)$is a polynomial in $\zeta$ and the quadratic form $q_{X}$ with coefficients involving only $\zeta^{2}$, homotopy invariants of $X$ (i.e. $b_{2}^{-}(X)$ ), and universal constants.

Our goal in this paper is to study the corresponding problem in algebraic geometry. More precisely, let $X$ be an algebraic surface (not necessarily with $\left.b_{2}^{+}(X)=1\right)$ and let $L$ be an ample line bundle on $X$. We can then identify the moduli space of $L$-stable rank two bundles $V$ on $X$ with $c_{1}(V)=\Delta$ and $c_{2}(V)=c$ with the moduli space of equivalence classes of ASD connections on $X$ with respect to a Hodge metric on $X$ corresponding to $L$. Let $\mathfrak{M}_{L}(\Delta, c)$ be the Gieseker compactification of this moduli space. It is known that $\mathfrak{M}_{L}(\Delta, c)$ changes as we change $L$, and that $\mathfrak{M}_{L}(\Delta, c)$ is constant on a set of chambers for the ample cone of $X$ which are defined in a way analogous to the definition of chambers for $\Omega_{X}$ given above. Using the recent result of Morgan [26] and Li [21] that the Donaldson polynomial of an algebraic 
surface can be evaluated using the Gieseker compactification $\mathfrak{M}_{L}(\Delta, c)$ of the moduli space of stable bundles, we shall work on $\mathfrak{M}_{L}(\Delta, c)$ for suitable choices of $L$ and in particular analyze the change in $\mathfrak{M}_{L}(\Delta, c)$ for $L \in \mathcal{C}_{+}$or $L \in \mathcal{C}_{-}$, where $\mathcal{C}_{ \pm}$are two adjacent chambers. It turns out that we can obtain $\mathfrak{M}_{L_{+}}(\Delta, c)$ from $\mathfrak{M}_{L_{-}}(\Delta, c)$ by a series of blowups and blowdowns (flips). Our results are thus very similar to those of Thaddeus in [33]. Thaddeus [34] and also Dolgachev-Hu [3] have developed a general picture for the variation of GIT quotients after a change of polarization, and although our methods are somewhat different it seems quite possible that they fit into their general framework. We have also found it convenient to borrow some of Thaddeus' notation.

Next we shall apply our results on the change in the moduli spaces to determine the transition formula for Donaldson polynomials in case $X$ is a rational surface with $-K_{X}$ effective. We shall give explicit formulas for $\delta_{w, p}^{X}\left(\mathcal{C}_{+}, \mathcal{C}_{-}\right)$in case the nonnegative integer $\ell_{\zeta}=\left(\zeta^{2}-p\right) / 4 \leq 2$. These formulas are in agreement with the above conjecture, in the sense that the transition formula is indeed a polynomial in $\zeta$ and $q_{X}$ with coefficients involving only universal constants, $\zeta^{2}$, and $K_{X}^{2}=9-b_{2}^{-}(X)=12-\chi(X)$ where $\chi(X)$ is the topological Euler-Poincare characteristic of $X$. We shall also give a formula in principle for $\delta_{w, p}^{X}\left(\mathcal{C}_{+}, \mathcal{C}_{-}\right)$in general (see Theorem 5.4), but to make this formula explicit involves more knowledge of the enumerative geometry of $\mathrm{Hilb}^{n} X$ than seems to be available at present. In case $-K_{X}$ is effective, the moduli spaces are (essentially) smooth and the centers of the blowup are smooth as well; in fact they are $\mathbb{P}^{k}$-bundles over Hilb $^{n_{1}} X \times$ Hilb $^{n_{2}} X$ for appropriate $k, n_{1}$ and $n_{2}$. In this way, we obtain general formulas which can be made explicit for low values of $n$. For instance, adopting Kotschick's notation [17]

$$
\varepsilon(\zeta, \Delta)=(-1)^{\frac{(\zeta-\Delta) \cdot K_{X}}{2}}=(-1)^{\left(\frac{\zeta-\Delta}{2}\right)^{2}}
$$

we show the following (see Theorem 6.4 for details):

Theorem. Assume that the wall $W^{\zeta}$ is defined only by $\pm \zeta$ with $\ell_{\zeta}=1$ and that $\mathcal{C}_{ \pm}$lies on the \pm -side of $W^{\zeta}$. Then, on the subspace of the symmetric algebra generated by $H_{2}(X), \delta_{w, p}^{X}\left(\mathcal{C}_{-}, \mathcal{C}_{+}\right)$is equal to

$$
(-1)^{d+1} \cdot \varepsilon(\zeta, \Delta) \cdot\left\{d(d-1) \cdot\left(\frac{\zeta}{2}\right)^{d-2} \cdot q_{X}+\left(2 K_{X}^{2}+2 d+6\right) \cdot\left(\frac{\zeta}{2}\right)^{d}\right\}
$$

Along the direction of the work of Kronheimer and Mrowka [19, 20], we also consider the difference of Donaldson polynomial invariants involving the 
natural generator $x \in H_{0}(X ; \mathbb{Z})$. More precisely, let $\nu$ be the corresponding 4-dimensional class in the instanton moduli space. For $\alpha \in H_{2}(X ; \mathbb{Z})$, we give a formula for the difference $\delta_{w, p}^{X}\left(\mathcal{C}_{-}, \mathcal{C}_{+}\right)\left(\alpha^{d-2}, \nu\right)$ in Theorem 5.5. It is worthwhile to point out that the similarity between Theorem 5.4 and Theorem 5.5 may indicate that there exists a deep relation between $\delta_{w, p}^{X}\left(\mathcal{C}_{-}, \mathcal{C}_{+}\right)\left(\alpha^{d}\right)$ and $\delta_{w, p}^{X}\left(\mathcal{C}_{-}, \mathcal{C}_{+}\right)\left(\alpha^{d-2}, \nu\right)$, and suggest a way to generalize the notion of simple type in $[19,20]$ from the case of $b_{2}^{+}>1$ to the case of $b_{2}^{+}=1$. For instance, modulo some lower degree terms, $\delta_{w, p}^{X}\left(\mathcal{C}_{-}, \mathcal{C}_{+}\right)\left(\alpha^{d-2}, \nu\right)$ can be obtained from $(-1 / 4) \cdot \delta_{w, p}^{X}\left(\mathcal{C}_{-}, \mathcal{C}_{+}\right)\left(\alpha^{d}\right)$ by replacing $d$ by $(d-2)$ (see Theorem 5.13 and Theorem 5.14). In fact, based on some heuristic arguments, it seems reasonable to conjecture that $\delta_{w, p}^{X}\left(\mathcal{C}_{-}, \mathcal{C}_{+}\right)\left(\alpha^{d-2}, \nu\right)$ is a combination of $\delta_{w, p^{\prime}}^{X}\left(\mathcal{C}_{-}, \mathcal{C}_{+}\right)\left(\alpha^{d-4 k}\right)$ for various nonnegative integers $k$ if the degrees are properly arranged.

Our paper is organized as follows. In section 2, we study rank two torsion free sheaves which are semistable with respect to ample divisors in $\mathcal{C}_{-}$but not semistable with respect to ample divisors in $\mathcal{C}_{+}$. When the surface $X$ is rational with $-K_{X}$ effective, these sheaves are parametrized by an open subset of a union of projective bundles over the product of two Hilbert schemes of points in $X$. More precisely, if $\zeta$ defines the wall separating $\mathcal{C}_{-}$from $\mathcal{C}_{+}$, define $E_{\zeta}^{n_{1}, n_{2}}$ be the set of all isomorphism classes of nonsplit extensions of the form

$$
0 \rightarrow \mathcal{O}_{X}(F) \otimes I_{Z_{1}} \rightarrow V \rightarrow \mathcal{O}_{X}(\Delta-F) \otimes I_{Z_{2}} \rightarrow 0,
$$

where $F$ is a divisor class such that $2 F-\Delta \equiv \zeta$ and $Z_{1}$ and $Z_{2}$ are two zerodimensional subschemes of $X$ with $\ell\left(Z_{i}\right)=n_{i}$ such that $n_{1}+n_{2}=\ell_{\zeta}$. In case $X$ is rational, $E_{\zeta}^{n_{1}, n_{2}}$ is a $\mathbb{P}^{N}$ bundle over $\operatorname{Hilb}^{n_{1}} X \times \operatorname{Hilb}^{n_{2}} X$, and the set of points of $E_{\zeta}^{n_{1}, n_{2}}$ lying in $\mathfrak{M}_{L_{-}}(\Delta, c)$ but not in $\mathfrak{M}_{L_{+}}(\Delta, c)$ is a Zariski open subset of $E_{\zeta}^{n_{1}, n_{2}}$. The main technical difficulty is that it is hard to control the rational map from $E_{\zeta}^{n_{1}, n_{2}}$ to $\mathfrak{M}_{L_{-}}(\Delta, c)$, and in particular this map is not a morphism. The general picture that we establish is the following: first, the map $E_{\zeta}^{0, \ell_{\zeta}} \rightarrow \mathfrak{M}_{L_{-}}(\Delta, c)$ is a morphism, and it is possible to make an elementary transformation, or flip, along its image. The result is a new space for which the rational map $E_{\zeta}^{1, \ell_{\zeta}-1} \rightarrow \mathfrak{M}_{L_{-}}(\Delta, c)$ becomes a morphism, and it is possible to make a flip along its image. We continue in this way until we reach $\mathfrak{M}_{L_{+}}(\Delta, c)$.

It seems rather difficult to see that the above picture holds directly. Instead we shall proceed as follows. We define abstractly a sequence of moduli spaces, indexed by an integer $k$ with $0 \leq k \leq \ell_{\zeta}+1$, such that the moduli space for $k=0$ is $\mathfrak{M}_{L_{-}}(\Delta, c)$, the moduli space for $k=\ell_{\zeta}+1$ is 
$\mathfrak{M}_{L_{+}}(\Delta, c)$, and moreover the $k^{\text {th }}$ moduli space contains an embedded copy of $E_{\zeta}^{k, \ell_{\zeta}-k}$ such that the flip along this copy yields the $(k+1)^{\text {st }}$ moduli space. Thus the picture is very similar to that developed independently by Thaddeus in [33]. To define our sequence of moduli spaces, we define $\left(L_{0}, \boldsymbol{\zeta}, \mathbf{k}\right)$-semistability in section 3 for rank two torsion free sheaves, where $L_{0}$ is any ample divisor contained in the common face of $\mathcal{C}_{+}$and $\mathcal{C}_{-}, \zeta$ is the set of classes of type $(\Delta, c)$ defining the common wall of $\mathcal{C}_{+}$and $\mathcal{C}_{-}$, and $\mathbf{k}$ is a set of integers. We show that $\mathfrak{M}_{L_{-}}(\Delta, c)$ and $\mathfrak{M}_{L_{+}}(\Delta, c)$ are linked by the moduli spaces $\mathfrak{M}_{0}^{(\zeta, \mathbf{k})}$ where the data $\mathbf{k}$ is allowed to vary. When the surface $X$ is rational with $-K_{X}$ effective, we can obtain $\mathfrak{M}_{L_{+}}(\Delta, c)$ from $\mathfrak{M}_{L_{-}}(\Delta, c)$ by a series of flips. The fact that all $\left(L_{0}, \boldsymbol{\zeta}, \mathbf{k}\right)$-semistable rank two torsion free sheaves do form a moduli space $\mathfrak{M}_{0}^{(\boldsymbol{\zeta}, \mathbf{k})}$ in the usual sense is proved in section 4 where we introduce an equivalent notion of stability called mixed stability. Our method follows Gieseker's GIT argument in [13]. Roughly speaking, the goal of mixed stability is to define stability for a sheaf of the form $V \otimes \Xi$, where $V$ is a torsion free sheaf but $\Xi$ is just a $\mathbb{Q}$-divisor. To make this idea precise, given actual divisors $H_{1}$ and $H_{2}$ and positive weights $a_{1}$ and $a_{2}$, we shall define a notion of stability which "mixes" stability for $V \otimes H_{1}$ with stability for $V \otimes H_{2}$, together with weightings of the stability condition for $V \otimes H_{i}$. The effect of this definition will be formally the same as if we had defined stability of $V \otimes \Xi$, where $\Xi$ is the $\mathbb{Q}$-divisor

$$
\frac{a_{1}}{a_{1}+a_{2}} H_{1}+\frac{a_{2}}{a_{1}+a_{2}} H_{2} \text {. }
$$

After the first draft of this paper was completed, we learned from the preprint of Ellingsrud and Göttsche [8] that the existence of the relevant mixed moduli spaces follows from more general results of Maruyama and Yokogawa [23, 38]. They constructed coarse moduli spaces for semistable torsion free parabolic sheaves over general smooth projective varieties, using results of Simpson [32]. However, the proof given here for the special case that we need is considerably shorter than the general proof and is based on different ideas, and so will be given in full.

In section 5 , using our results on flips of moduli spaces, we give a formula for the transition formula of Donaldson polynomials when $X$ is rational with $-K_{X}$ effective, and compute the leading term in the transition formula. In section 6 , we obtain explicit transition formulas when $\ell_{\zeta} \leq 2$.

Some of the material in our section 2 has been worked out independently by $\mathrm{Hu}$ and Li [16] and Göttsche [14]. Moreover Ellingsrud and Göttsche [8] have recently studied the change in the moduli space by similar methods and 
have obtained results very similar to ours. Using very different methods, the results in Section 4 have also been obtained by Matsuki and Wentworth [24], who also consider the case of higher rank. They use branched covers of the surface $X$ to study the change in the moduli space. We expect that a minor modification of the arguments in Section 4 of this paper will also handle the case of higher rank.

\section{Conventions and notations.}

We fix some conventions and notations for the rest of this paper. Let $X$ be a smooth algebraic surface. We shall be primarily interested in the case where $X$ is simply connected and $-K_{X}$ is effective and nonzero. Thus necessarily $X$ is a rational surface. However much of the discussion in sections 1-4 will also apply to the general case. Stability and semistability with respect to an ample line bundle $L$ will always be understood to mean Gieseker stability or semistability unless otherwise noted. We shall not mention the choice of $L$ explicitly if it is clear from the context. Recall that a torsion free sheaf $V$ of rank two is Gieseker $L$-stable if and only if, for every rank one subsheaf $W$ of $V$, either $\mu_{L}(W)<\mu_{L}(V)$ or $\mu_{L}(W)=\mu_{L}(V)$ and $2 \chi(W)<\chi(V)$, where $\mu_{L}$ is the normalized degree with respect to $L$. Semistability is similarly defined, where the second inequality is also allowed to be an equality. For a torsion free sheaf $V$, we use $V^{\vee \vee}$ to stand for its double dual. For two divisors $D_{1}$ and $D_{2}$ on $X$, the notation $D_{1} \equiv D_{2}$ means that $D_{1}$ and $D_{2}$ are numerically equivalent, that is, $D_{1} \cdot D=D_{2} \cdot D$ for any divisor $D$. For a locally free sheaf (or equivalently a vector bundle) $\mathcal{E}$ over a smooth variety $Y$, we use $\mathbb{P}(\mathcal{E})$ to denote the associated projective space bundle, that is, $\mathbb{P}(\mathcal{E})$ is the Proj of $\oplus_{d \geq 0} S^{d}(\mathcal{E})$.

Fix a divisor $\Delta$ and an integer $c$. Let $\mathcal{C}_{-}$and $\mathcal{C}_{+}$be two adjacent chambers of type $(\Delta, c)$ separated by the wall $W^{\zeta}$. We assume that $\zeta \cdot \mathcal{C}_{-}<0<\zeta \cdot \mathcal{C}_{+}$. Let $L_{ \pm} \in \mathcal{C}_{ \pm}$be an ample line bundle, so that $L_{-} \cdot \zeta<0<L_{+} \cdot \zeta$, and denote by $\mathfrak{M}_{ \pm}$the moduli space $\mathfrak{M}_{L_{ \pm}}(\Delta, c)$ of rank two Gieseker semistable torsion free sheaves $V$ with $c_{1}(V)=\Delta$ and $c_{2}(V)=c$. Let $L_{0}$ be any ample divisor contained in the interior of the intersection of $W^{\zeta}$ and the closures of $\mathcal{C}_{ \pm}$. Let $\zeta=\zeta_{1}, \ldots, \zeta_{n}$ be all the positive rational multiples of $\zeta$ such that $\zeta_{i}$ is an integral class of type $(w, p)$ which also defines the wall $W^{\zeta}$. In sections $5-6$, we will assume that $n=1$ for notational simplicity.

Finally, we point out that our $\mu$-map is half of the $\mu$-map used in $[17,18]$ (see (viii) and (ix) in Notation 5.1). Thus our transition formula differs from the one defined in [18] by a universal constant. 
Acknowledgements. We would like to thank Hong-Jie Yang for invaluable access to his calculations, which helped to keep us on the right track. The second author would like to thank Lothar Goettsche, Wei-ping Li, and YunGang Ye for helpful discussions, and the Institute for Advanced Study at Princeton for its hospitality and financial support through NSF grant DMS9100383 during the academic year 1992-1993 when part of this work was done.

\section{Preliminaries on the moduli space.}

In this section, we study rank two torsion free sheaves which are related to walls. These sheaves arise naturally from the comparison of $L_{-}-$ semistability and $L_{+}$-semistability. We will show that when the surface $X$ is rational with $-K_{X}$ effective, the moduli spaces $\mathfrak{M}_{ \pm}$are smooth at the points corresponding to these sheaves. We start with the following lemma, which for simplicity is just stated for $L_{-}$-stability.

Lemma 2.1. Let $V$ be a rank two torsion free sheaf on $X$ with $c_{1}(V)=\Delta$ and $c_{2}(V)=c$. If $V$ is $L_{-}$-semistable, then exactly one of the following holds:

(i) Both $V$ and $V^{\vee \vee}$ are $L_{-}$-stable and Mumford $L_{-}$-stable.

(ii) $V$ sits in an exact sequence

$$
0 \rightarrow \mathcal{O}_{X}\left(F_{1}\right) \otimes I_{Z_{1}} \rightarrow V \rightarrow \mathcal{O}_{X}\left(F_{2}\right) \otimes I_{Z_{2}} \rightarrow 0
$$

where $2 F_{1} \equiv \Delta \equiv 2 F_{2}$, and $Z_{1}$ and $Z_{2}$ are zero-dimensional subschemes of $X$ such that $\ell\left(Z_{1}\right) \geq \ell\left(Z_{2}\right)$. Moreover in this case $V$ is $L$-semistable for every choice of an ample line bundle $L$ and $V$ is strictly $L_{ \pm}$-semistable if and only if $\ell\left(Z_{1}\right)=\ell\left(Z_{2}\right)$.

Proof. Suppose that $V$ is (Gieseker) $L_{-}$-semistable. The vector bundle $V^{\vee \vee}$ satisfies $c_{1}\left(V^{\vee \vee}\right)=\Delta$ and $c_{2}\left(V^{\vee \vee}\right) \leq c$. Standard arguments [10] show that $V^{\vee \vee}$ is Mumford $L_{-}$-semistable. If $V^{\vee \vee}$ is strictly Mumford $L_{-}$-semistable, then by $[10,31]$, either $L_{-}$must lie on a wall of type $(\Delta, c)$ or if $\mathcal{O}_{X}\left(F_{1}\right)$ is a destabilizing sub-line bundle then $\Delta \equiv 2 F_{1}$. Since by assumption $L_{-}$does not lie on a wall of type $(\Delta, c)$, either $V^{\vee \vee}$ is Mumford $L_{-}$-stable or there is an exact sequence

$$
0 \rightarrow \mathcal{O}_{X}\left(F_{1}\right) \rightarrow V^{\vee \vee} \rightarrow \mathcal{O}_{X}\left(F_{2}\right) \otimes I_{Z} \rightarrow 0
$$


where $F_{2}=\Delta-F_{1} \equiv F_{1}$ and $Z$ is a zero-dimensional subscheme of $X$. If $V^{\vee \vee}$ is Mumford $L_{-}$-stable, then $V$ is Mumford $L_{-}$-stable and therefore $L_{-}$stable. Thus case (i) holds. Otherwise $\mathcal{O}_{X}\left(F_{1}\right) \cap V$ is of the form $\mathcal{O}_{X}\left(F_{1}\right) \otimes$ $I_{Z_{1}}$ for some $Z_{1}$ and $V / \mathcal{O}_{X}\left(F_{1}\right) \otimes I_{Z_{1}}$ is a subsheaf of $\mathcal{O}_{X}\left(F_{2}\right) \otimes I_{Z}$ and thus of the form $\mathcal{O}_{X}\left(F_{2}\right) \otimes I_{Z_{2}}$ for some $Z_{2}$. Thus we are in case (ii) of the lemma. Since $\mu\left(\mathcal{O}_{X}\left(F_{1}\right) \otimes I_{Z_{1}}\right)=\mu(V)$ and $V$ is semistable, we have

$$
2 \chi\left(\mathcal{O}_{X}\left(F_{1}\right) \otimes I_{Z_{1}}\right) \leq \chi(V)=\chi\left(\mathcal{O}_{X}\left(F_{1}\right) \otimes I_{Z_{1}}\right)+\chi\left(\mathcal{O}_{X}\left(F_{2}\right) \otimes I_{Z_{2}}\right) .
$$

Hence $\chi\left(\mathcal{O}_{X}\left(F_{2}\right) \otimes I_{Z_{2}}\right)-\chi\left(\mathcal{O}_{X}\left(F_{1}\right) \otimes I_{Z_{1}}\right) \geq 0$. As $F_{1} \equiv F_{2}$ and $\chi\left(\mathcal{O}_{X}\left(F_{i}\right) \otimes\right.$ $\left.I_{Z_{i}}\right)=\chi\left(\mathcal{O}_{X}\left(F_{i}\right)\right)-\ell\left(Z_{i}\right)$, we must then have $\ell\left(Z_{1}\right)-\ell\left(Z_{2}\right) \geq 0$. The last sentence of (ii) is a straightforward argument left to the reader.

If $V$ satisfies the conclusions of (2.1)(ii), we shall call $V$ universally semistable.

Next we shall compare stability for $L_{-}$and $L_{+}$.

Lemma 2.2. Let $V$ be a torsion free rank two sheaf with $c_{1}(V)=\Delta$ and $c_{2}(V)=c$.

(i) If $V$ is $L_{-}$-stable but $L_{+}$-unstable, then there exist a divisor class $F$ and two zero-dimensional subschemes $Z_{-}$and $Z_{+}$of $X$ and an exact sequence

$$
0 \rightarrow \mathcal{O}_{X}(F) \otimes I_{Z_{-}} \rightarrow V \rightarrow \mathcal{O}_{X}(\Delta-F) \otimes I_{Z_{+}} \rightarrow 0,
$$

where $L_{-} \cdot(2 F-\Delta)<0<L_{+} \cdot(2 F-\Delta)$. Moreover the divisor $F$, the schemes $Z_{-}$and $Z_{+}$, and the map $F \otimes I_{Z_{-}} \rightarrow V$ are unique mod scalars, and $\zeta=2 F-\Delta$ defines a wall of type $(\Delta, c)$.

(ii) Conversely, suppose that there is a nonsplit exact sequence as above. Then $V$ is simple. Moreover, $V$ is not $L_{-}$-stable if and only if it is $L_{-}$-unstable if and only if there exist subschemes $Z^{\prime}$ and $Z^{\prime \prime}$ and an exact sequence

$$
0 \rightarrow \mathcal{O}_{X}(\Delta-F) \otimes I_{Z^{\prime}} \rightarrow V \rightarrow \mathcal{O}_{X}(F) \otimes I_{Z^{\prime \prime}} \rightarrow 0,
$$

if and only if $V^{\vee \vee}$ is a direct sum $\mathcal{O}_{X}(F) \oplus \mathcal{O}_{X}(\Delta-F)$. In this case the scheme $Z^{\prime}$ strictly contains the scheme $Z_{+}, \ell\left(Z^{\prime}\right)>\ell\left(Z_{+}\right)$and $\ell\left(Z^{\prime}\right)+\ell\left(Z^{\prime \prime}\right)=\ell\left(Z_{-}\right)+\ell\left(Z_{+}\right)$. Finally, if $Z_{-}=\emptyset$ then $V$ is always $L_{-}$-stable. 
Proof. We first show (i). Suppose that $V$ is $L_{-}$-stable but $L_{+}$-unstable. Then by (2.1) $V^{\vee \vee}$ is also $L_{-}$-stable and $L_{+}$-unstable. By [31], there is a uniquely determined line bundle $\mathcal{O}_{X}(F)$ and a map $\mathcal{O}_{X}(F) \rightarrow V^{\vee \vee}$ with torsion free quotient such that $L_{-} \cdot(2 F-\Delta)<0<L_{+} \cdot(2 F-\Delta)$. Moreover $\zeta=2 F-\Delta$ defines a wall of type $(\Delta, c)$. The subsheaf $\mathcal{O}_{X}(F) \cap V$ of $V^{\vee \vee}$ is a subsheaf of $\mathcal{O}_{X}(F)$ and agrees with it away from finitely many points. Thus $\mathcal{O}_{X}(F) \cap V=\mathcal{O}_{X}(F) \otimes I_{Z_{-}}$for some well-defined subscheme $Z_{-}$. Moreover the quotient $V /\left[\mathcal{O}_{X}(F) \otimes I_{Z_{-}}\right]$is a subsheaf of $\mathcal{O}_{X}(\Delta-F) \otimes I_{Z}$ for some zero-dimensional subscheme $Z$, and agrees with $\mathcal{O}_{X}(\Delta-F)$ away from finitely many points. Thus the quotient is of the form $\mathcal{O}_{X}(\Delta-F) \otimes I_{Z_{+}}$ for some zero-dimensional subscheme $Z_{+}$. The uniqueness is clear.

To see (ii), suppose that $V$ is given as a nonsplit exact sequence

$$
0 \rightarrow \mathcal{O}_{X}(F) \otimes I_{Z_{-}} \rightarrow V \rightarrow \mathcal{O}_{X}(\Delta-F) \otimes I_{Z_{+}} \rightarrow 0
$$

as above, where $L_{-} \cdot(2 F-\Delta)<0<L_{+} \cdot(2 F-\Delta)$. Again by $(2.1), V$ is $L_{-}-$ semistable if and only if it is $L_{-}$-stable if and only if $V^{\vee V}$ is $L_{-}$-stable. Now taking double duals of the above exact sequence, there is an exact sequence

$$
0 \rightarrow \mathcal{O}_{X}(F) \rightarrow V^{\vee \vee} \rightarrow \mathcal{O}_{X}(\Delta-F) \otimes I_{Z} \rightarrow 0
$$

for some zero-dimensional scheme $Z$. Moreover, by [31], $V^{\vee \vee}$ is $L_{-}$-unstable if and only if the above exact sequence splits, and in particular if and only if $Z=\emptyset$ and $V^{\vee \vee}=\mathcal{O}_{X}(F) \oplus \mathcal{O}_{X}(\Delta-F)$. In this case, the map $\mathcal{O}_{X}(\Delta-F) \rightarrow$ $V^{\vee \vee}$ induces a map $\mathcal{O}_{X}(\Delta-F) \otimes I_{Z^{\prime}} \rightarrow V$ for some ideal sheaf $I_{Z^{\prime}}$. We may clearly assume that the quotient is torsion free, in which case it is necessarily of the form $\mathcal{O}_{X}(F) \otimes I_{Z^{\prime \prime}}$ with $\ell\left(Z^{\prime}\right)+\ell\left(Z^{\prime \prime}\right)=\ell\left(Z_{-}\right)+\ell\left(Z_{+}\right)$. Using the nonzero map $\mathcal{O}_{X}(\Delta-F) \otimes I_{Z^{\prime}} \rightarrow \mathcal{O}_{X}(\Delta-F) \otimes I_{Z_{+}}$, we see that there is an inclusion $I_{Z^{\prime}} \subseteq I_{Z_{+}}$; moreover this inclusion must be strict since the defining exact sequence for $V$ is nonsplit. Thus $Z^{\prime}$ strictly contains $Z_{+}$ and in particular $\ell\left(Z^{\prime}\right)>\ell\left(Z_{+}\right)$. Conversely, if there exists a nonzero map $\mathcal{O}_{X}(\Delta-F) \otimes I_{Z^{\prime}} \rightarrow V$, then there is a nonzero map $\mathcal{O}_{X}(\Delta-F) \rightarrow V^{\vee \vee}$ and thus $V^{\vee \vee}$ is the split extension.

We next show that $V$ is simple. If $V$ is stable then it is simple. If $V$ is not stable, then $V^{\vee \vee}=\mathcal{O}_{X}(F) \oplus \mathcal{O}_{X}(\Delta-F)$. There is an inclusion $\operatorname{Hom}(V, V) \subseteq \operatorname{Hom}\left(V^{\vee \vee}, V^{\vee \vee}\right)$. If $V^{\vee \vee}$ is split, then $\operatorname{Hom}\left(V^{\vee \vee}, V^{\vee \vee}\right)=$ $\mathbb{C} \oplus \mathbb{C}$. In this case, using a nonscalar endomorphism of $V$, it is easy to see that we can split the exact sequence defining $V$.

Finally suppose that $Z_{-}=\emptyset$ in the notation of (2.2). If $V$ is $L_{-}-$ unstable, then we can find $Z^{\prime}$ with $\ell\left(Z^{\prime}\right)>\ell\left(Z_{+}\right)$and a subscheme $Z^{\prime \prime}$ such 
that $\ell\left(Z^{\prime}\right)+\ell\left(Z^{\prime \prime}\right)=\ell\left(Z_{+}\right)$. Thus $\ell\left(Z^{\prime}\right) \leq \ell\left(Z_{+}\right)$, a contradiction. It follows that $V$ is $L_{-}$-stable.

For the rest of this section, we shall assume that $-K_{X}$ is effective and nonzero and that $q(X)=0$. Thus $X$ is a rational surface.

Lemma 2.3. Suppose that $\mathfrak{M}_{ \pm}$is nonempty. Suppose that $(w, p) \neq(0,0)$, or equivalently that $\mathfrak{M}_{ \pm}$does not consist of a single point corresponding to a twist of the trivial vector bundle. Then the open subset of $\mathfrak{M}_{ \pm}$corresponding to Mumford stable rank two vector bundles is nonempty and dense. Every component of $\mathfrak{M}_{ \pm}$has dimension $4 c-\Delta^{2}-3=-p-3$. The points of $\mathfrak{M}_{ \pm}$ corresponding to $L_{ \pm}$-stable sheaves $V$ are smooth points.

Proof. Suppose that $\mathfrak{M}_{ \pm}$is nonempty, and let $V$ correspond to a point of $\mathfrak{M}_{ \pm}$. Then by general theory (e.g. Chapter 7 of [10]), $\mathfrak{M}_{ \pm}$is smooth of dimension $4 c-\Delta^{2}-3=-p-3$ at $V$ if $V$ is stable and $\operatorname{Ext}^{2}(V, V)=0$, since $h^{2}\left(X ; \mathcal{O}_{X}\right)=0$. Moreover, setting $W=V^{\vee \vee}$, there is a surjection from $H^{2}(X ; H o m(W, W))$ to $\operatorname{Ext}^{2}(V, V)$. Thus to show that $\operatorname{Ext}^{2}(V, V)=0$ it suffices to show that $H^{2}(X ; \operatorname{Hom}(W, W))=0$. Now $H^{2}(X ; \operatorname{Hom}(W, W))$ is dual to $H^{0}\left(X ; \operatorname{Hom}(W, W) \otimes K_{X}\right)$. Since $-K_{X}$ is effective, there is an inclusion of $H^{0}\left(X ; H o m(W, W) \otimes K_{X}\right)$ in $H^{0}(X ; H o m(W, W))$. If $W$ is stable, then $H^{0}(X ; H o m(W, W)) \cong \mathbb{C}$ and $H^{0}\left(X ; H o m(W, W) \otimes K_{X}\right)=0$. Thus $\mathfrak{M}_{ \pm}$is smooth at $V$. Standard theory $[1,10]$ also shows that every torsion free sheaf $V$ for which $V^{\vee V}$ is stable is smoothable. Thus the set of locally free sheaves is nonempty and dense in the component containing $V$ in this case.

Now consider a $V$ such that $W=V^{\vee \vee}$ is not stable. Using the exact sequence

$$
0 \rightarrow \mathcal{O}_{X}(F) \rightarrow W \rightarrow \mathcal{O}_{X}(F) \otimes I_{Z} \rightarrow 0
$$

for $W$ which was given in the course of the proof of (2.1), it is easy to check that there is an exact sequence

$$
\begin{aligned}
0 \rightarrow \operatorname{Hom}\left(I_{Z}, W \otimes \mathcal{O}_{X}(-F) \otimes K_{X}\right) \rightarrow \operatorname{Hom}\left(W, W \otimes K_{X}\right) & \rightarrow \\
& \rightarrow H^{0}\left(W \otimes \mathcal{O}_{X}(-F) \otimes K_{X}\right) .
\end{aligned}
$$

Since $-K_{X}$ is effective and nonzero, $H^{0}\left(W \otimes \mathcal{O}_{X}(-F) \otimes K_{X}\right)=$ $\operatorname{Hom}\left(I_{Z}, W \otimes \mathcal{O}_{X}(-F) \otimes K_{X}\right)=0$. Thus $\operatorname{Hom}\left(W, W \otimes K_{X}\right)=0$ as well. Once again $V$ is smoothable. 
Now we claim that a general smoothing $V^{\prime}$ of $V$ is Mumford stable. For otherwise by the proof of (2.1) there is an exact sequence

$$
0 \rightarrow \mathcal{O}_{X}(F) \rightarrow V^{\prime} \rightarrow \mathcal{O}_{X}(F) \otimes I_{Z} \rightarrow 0
$$

as above, with $\ell(Z) \leq \ell(\emptyset)=0$. In this case $V^{\prime}$ is an extension of $\mathcal{O}_{X}(F)$ by $\mathcal{O}_{X}(F)$, forcing $w=p=0$ and (since $\left.h^{1}\left(\mathcal{O}_{X}\right)=0\right) V^{\prime}=\mathcal{O}_{X}(F) \oplus \mathcal{O}_{X}(F)$.

It is natural to make the following conjecture, which is true for geometrically ruled $X$ by [30] and is verified in certain other cases by [36].

Conjecture 2.4. If $X$ is a rational surface with $-K_{X}$ effective, then for every choice of $L, \Delta$ and $c, \mathfrak{M}_{L}(\Delta, c)$ is either empty or irreducible.

Let us fix some notations for the rest of this paper.

Definition 2.5. Let $X$ be an algebraic surface (not necessarily rational), and let $\zeta$ be a fixed numerical equivalence class defining a wall of type $(\Delta, c)$. Set $\ell_{\zeta}=\left(4 c-\Delta^{2}+\zeta^{2}\right) / 4=\left(\zeta^{2}-p\right) / 4$. Choose two nonnegative integers $n_{-}$and $n_{+}$with $n_{-}+n_{+}=\ell_{\zeta}$, and let $E_{\zeta}^{n_{-}, n_{+}}$be the set of all isomorphism classes of nonsplit extensions of the form

$$
0 \rightarrow \mathcal{O}_{X}(F) \otimes I_{Z_{-}} \rightarrow V \rightarrow \mathcal{O}_{X}(\Delta-F) \otimes I_{Z_{+}} \rightarrow 0
$$

with $\zeta \equiv 2 F-\Delta$ and $\ell\left(Z_{ \pm}\right)=n_{ \pm}$.

We remark that since $\zeta \equiv \Delta(\bmod 2)$ and $\Delta^{2}-4 c \leq \zeta^{2}<0, \ell_{\zeta}$ is a nonnegative integer. If $V$ corresponds to a point of $E_{\zeta}^{n_{-}, n_{+}}$, then $V$ is $L_{+-}$ unstable since $L_{+} \cdot \zeta>0$. By (2.2)(ii), $V$ is simple, and if it is $L_{-}$-semistable then it is actually stable. By (2.3), if $X$ is a rational surface with $-K_{X}$ effective, then $\mathfrak{M}_{-}$is smooth in a neighborhood of a point corresponding to a sheaf $V$ lying in $E_{\zeta}^{n_{-}, n_{+}}$for some $\zeta, n_{-}, n_{+}$. We shall now study $E_{\zeta}^{n_{-}, n_{+}}$ in more detail for rational surfaces.

Lemma 2.6. Suppose that $-K_{X}$ is effective and that $q(X)=0$. For $Z_{-}$ and $Z_{+}$two fixed zero-dimensional subschemes of $X$ of lengths $n_{-}$and $n_{+}$ respectively,

$\operatorname{dim} \operatorname{Ext}^{1}\left(\mathcal{O}_{X}(\Delta-F) \otimes I_{Z_{+}}, \mathcal{O}_{X}(F) \otimes I_{Z_{-}}\right)=n_{-}+n_{+}+h(\zeta)=\ell_{\zeta}+h(\zeta)$, where

$$
h(\zeta)=h^{1}\left(X ; \mathcal{O}_{X}(2 F-\Delta)\right)=\frac{\left(\zeta \cdot K_{X}\right)}{2}-\frac{\zeta^{2}}{2}-1
$$


Proof. Note that $\operatorname{Hom}\left(\mathcal{O}_{X}(\Delta-F) \otimes I_{Z_{+}}, \mathcal{O}_{X}(F) \otimes I_{Z_{-}}\right) \subseteq H^{0}\left(\mathcal{O}_{X}(2 F-\Delta)\right)=$ 0 , since $L_{-} \cdot(2 F-\Delta)<0$. Likewise $\operatorname{Ext}^{2}\left(\mathcal{O}_{X}(\Delta-F) \otimes I_{Z_{+}}, \mathcal{O}_{X}(F) \otimes I_{Z_{-}}\right)$ is Serre dual to $\operatorname{Hom}\left(\mathcal{O}_{X}(F) \otimes I_{Z_{-}}, \mathcal{O}_{X}(\Delta-F) \otimes I_{Z_{+}} \otimes K_{X}\right) \subseteq H^{0}\left(\mathcal{O}_{X}(\Delta-\right.$ $\left.2 F) \otimes K_{X}\right) \subseteq H^{0}\left(\mathcal{O}_{X}(\Delta-2 F)\right)$, since $-K_{X}$ is effective. Thus as $L_{+}$. $(\Delta-2 F)<0, \operatorname{Ext}^{2}\left(\mathcal{O}_{X}(\Delta-F) \otimes I_{Z_{+}}, \mathcal{O}_{X}(F) \otimes I_{Z_{-}}\right)=0$ as well. If we set $\chi\left(\mathcal{O}_{X}(\Delta-F) \otimes I_{Z_{+}}, \mathcal{O}_{X}(F) \otimes I_{Z_{-}}\right)=\sum_{i}(-1)^{i} \operatorname{dim} \operatorname{Ext}^{i}\left(\mathcal{O}_{X}(\Delta-F) \otimes\right.$ $\left.I_{Z_{+}}, \mathcal{O}_{X}(F) \otimes I_{Z_{-}}\right)$, then

$$
\begin{aligned}
\chi\left(\mathcal{O}_{X}(\Delta-F) \otimes I_{Z_{+}}, \mathcal{O}_{X}(F) \otimes I_{Z_{-}}\right) & = \\
& =-\operatorname{dim} \operatorname{Ext}^{1}\left(\mathcal{O}_{X}(\Delta-F) \otimes I_{Z_{+}}, \mathcal{O}_{X}(F) \otimes I_{Z_{-}}\right) .
\end{aligned}
$$

Now a standard argument [28] shows that

$$
\begin{gathered}
\chi\left(\mathcal{O}_{X}(\Delta-F) \otimes I_{Z_{+}}, \mathcal{O}_{X}(F) \otimes I_{Z_{-}}\right) \\
=\int_{X} \operatorname{ch}\left(\mathcal{O}_{X}(\Delta-F) \otimes I_{Z_{+}}\right)^{\vee} \cdot \operatorname{ch}\left(\mathcal{O}_{X}(F) \otimes I_{Z_{-}}\right) \cdot \operatorname{Todd}_{X} .
\end{gathered}
$$

Here given a class $a=\sum a_{i} \in \bigoplus_{i} A^{i}(X)$, we denote by $a^{\vee}$ the class $\sum_{i}(-1)^{i} a_{i}$. An easy computation gives

$$
\begin{aligned}
& \int_{X} \operatorname{ch}\left(\mathcal{O}_{X}(\Delta-F) \otimes I_{Z_{+}}\right)^{\vee} \cdot \operatorname{ch}\left(\mathcal{O}_{X}(F) \otimes I_{Z_{-}}\right) \cdot \operatorname{Todd}_{X} \\
= & \int_{X} \operatorname{ch}\left(\mathcal { O } _ { X } ( \Delta - F ) ^ { \vee } \cdot \operatorname { c h } \left(\mathcal{O}_{X}(F) \cdot \operatorname{Todd}_{X}-\ell\left(Z_{-}\right)-\ell\left(Z_{+}\right) .\right.\right.
\end{aligned}
$$

Reversing the above argument, we see that

$$
\begin{aligned}
\int_{X} \operatorname{ch}\left(\mathcal { O } _ { X } ( \Delta - F ) ^ { \vee } \cdot \operatorname { c h } \left(\mathcal{O}_{X}(F) \cdot \operatorname{Todd}_{X}\right.\right. & =\chi\left(\mathcal{O}_{X}(2 F-\Delta)\right) \\
=-h^{1}\left(X ; \mathcal{O}_{X}(2 F-\Delta)\right) & =\frac{\zeta^{2}}{2}-\frac{\left(\zeta \cdot K_{X}\right)}{2}+1=-h(\zeta) .
\end{aligned}
$$

Putting these together we see that $\operatorname{dim} \operatorname{Ext}^{1}\left(\mathcal{O}_{X}(\Delta-F) \otimes I_{Z_{+}}, \mathcal{O}_{X}(F) \otimes I_{Z_{-}}\right)$ is equal to $n_{-}+n_{+}+h(\zeta)$.

Let us describe the scheme structure on $E_{\zeta}^{n_{-}, n_{+}}$more carefully. For $Z_{-}$ and $Z_{+}$fixed, the set of extensions in $E_{\zeta}^{n_{-}, n_{+}}$corresponding to $Z_{-}, Z_{+}$, is equal to $\mathbb{P} \operatorname{Ext}^{1}\left(\mathcal{O}_{X}(\Delta-F) \otimes I_{Z_{+}}, \mathcal{O}_{X}(F) \otimes I_{Z_{-}}\right)$. To make a universal construction, let $H_{n_{ \pm}}=$Hilb $^{n_{ \pm}} X$. Let $\mathcal{Z}_{n_{ \pm}}$be the universal codimension two subscheme of $X \times H_{n_{ \pm}}$. Let $\pi_{1}, \pi_{2}$ be the projections of $X \times H_{n_{-}} \times H_{n_{+}}$ 
to $X, H_{n_{-}} \times H_{n_{+}}$respectively, and let $\pi_{1,2}, \pi_{1,3}$ be the projections of $X \times$ $H_{n_{-}} \times H_{n_{+}}$to $X \times H_{n_{-}}, X \times H_{n_{+}}$respectively. Define

$$
\mathcal{E}_{\zeta}^{n_{-}, n_{+}}=\operatorname{Ext}_{\pi_{2}}^{1}\left(\pi_{1}^{*} \mathcal{O}_{X}(\Delta-F) \otimes \pi_{1,3}^{*} I_{\mathcal{Z}_{n_{+}}}, \pi_{1}^{*} \mathcal{O}_{X}(F) \otimes \pi_{1,2}^{*} I_{\mathcal{Z}_{n_{-}}}\right) .
$$

The previous lemma and standard base change results show that $\mathcal{E}_{\zeta}^{n_{-}, n_{+}}$is locally free of rank $h(\zeta)+\ell_{\zeta}$ over $H_{n_{-}} \times H_{n_{+}}$. We set $E_{\zeta}^{n_{-}, n_{+}}=\mathbb{P}\left(\left(\mathcal{E}_{\zeta}^{n_{-}, n_{+}}\right)^{\vee}\right)$, if $h(\zeta)+\ell_{\zeta}>0$. Moreover by standard facts about relative Ext sheaves there is an exact sequence

$$
\begin{aligned}
0 \rightarrow R^{1} \pi_{2 *} \operatorname{Hom}\left(\pi_{1}^{*} \mathcal{O}_{X}(\Delta-F) \otimes \pi_{1,3}^{*} I_{\mathcal{Z}_{n_{+}}}, \pi_{1}^{*} \mathcal{O}_{X}(F) \otimes \pi_{1,2}^{*} I_{\mathcal{Z}_{n_{-}}}\right) \rightarrow \\
\rightarrow \mathcal{E}_{\zeta}^{n_{-}, n_{+}} \rightarrow \\
\rightarrow \pi_{2 *} \operatorname{Ext}^{1}\left(\pi_{1}^{*} \mathcal{O}_{X}(\Delta-F) \otimes \pi_{1,3}^{*} I_{\mathcal{Z}_{n_{+}}}, \pi_{1}^{*} \mathcal{O}_{X}(F) \otimes \pi_{1,2}^{*} I_{\mathcal{Z}_{n_{-}}}\right) \rightarrow 0 .
\end{aligned}
$$

Corollary 2.7. With $X$ as in (2.6), if $h(\zeta)+\ell_{\zeta}=h^{1}\left(X ; \mathcal{O}_{X}(2 F-\Delta)+\ell_{\zeta} \neq\right.$

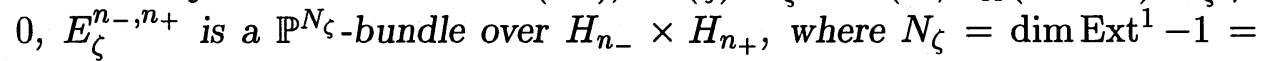
$h(\zeta)+\ell_{\zeta}-1$. Thus if $h(\zeta)+\ell_{\zeta} \neq 0$, then $\operatorname{dim} E_{\zeta}^{n_{-}, n_{+}}=3 \ell_{\zeta}+h(\zeta)-1$.

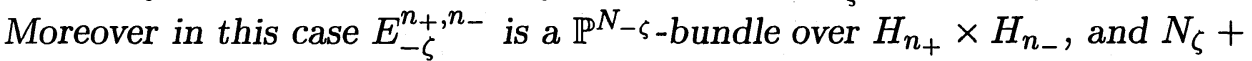
$N_{-\zeta}+2 \ell_{\zeta}=-p-4$. If $h(\zeta)+\ell_{\zeta}=0$, then $E_{\zeta}^{0,0}=\emptyset$ and $E_{-\zeta}^{0,0}=\mathbb{P}^{-p-3}$ is a component of $\mathfrak{M}_{+}$. Finally this last case arises if and only if $\zeta^{2}=p$ and $\zeta \cdot K_{X}=\zeta^{2}+2=p+2$.

Proof. Note that $N_{\zeta} \geq 0$ unless $h(\zeta)+\ell_{\zeta}=0$. Under this assumption, we have

$$
N_{\zeta}+N_{-\zeta}+2 \ell_{\zeta}=4 \ell_{\zeta}-\zeta^{2}-4=-p-4 .
$$

The case where $h(\zeta)+\ell_{\zeta}=0$ is similar. Moreover if $h(\zeta)+\ell_{\zeta}=0$, then it follows from (2.2)(ii) that all of the sheaves $V$ corresponding to points of $E_{-\zeta}^{0,0}$ are $L_{+}$-stable. By (2.2)(i) the map $E_{-\zeta}^{0,0} \rightarrow \mathfrak{M}_{+}$is one-to-one. Since $\mathfrak{M}_{+}$ is of dimension $-p-3$ and smooth at points corresponding to the sheaves in $E_{-\zeta}^{0,0} \rightarrow \mathfrak{M}_{+}$, the map $E_{-\zeta}^{0,0} \rightarrow \mathfrak{M}_{+}$must be an embedding onto a component of $\mathfrak{M}_{+}$. The final statement follows from the formulas $\zeta^{2}=4 \ell_{\zeta}+p$ and $h(\zeta)=\frac{\left(\zeta \cdot K_{X}\right)}{2}-\frac{\zeta^{2}}{2}-1$.

If $h(\zeta)+\ell_{\zeta} \neq 0$, then by Lemma 2.2 there is a rational map from $E_{\zeta}^{n_{-}, n_{+}}$ to the moduli space $\mathfrak{M}_{-}$which is birational onto its image. However this map will not in general be a morphism if $n_{-}>0$ (see [16]). We shall study this more carefully in the next sections. 
Let us also remark that standard theory gives a universal sheaf $\mathcal{V}$ over $E_{\zeta}^{n_{-}, n_{+}}$:

Proposition 2.8. Let $\rho: X \times E_{\zeta}^{n_{-}, n_{+}} \rightarrow X \times H_{n_{-}} \times H_{n_{+}}$be the natural projection, and let $\pi_{2}: X \times E_{\zeta}^{n_{-}, n_{+}} \rightarrow E_{\zeta}^{n_{-}, n_{+}}$be the projection. Then there is a coherent sheaf $\mathcal{V}$ over $X \times E_{\zeta}^{n_{-}, n_{+}}$and an exact sequence

$$
\begin{aligned}
0 & \rightarrow \rho^{*}\left(\pi_{1}^{*} \mathcal{O}_{X}(F) \otimes \pi_{1,2}^{*} I_{\mathcal{Z}_{n_{-}}}\right) \otimes \pi_{2}^{*} \mathcal{O}_{E_{\zeta}^{n_{-}, n_{+}}}(1) \\
& \rightarrow \mathcal{V} \rightarrow \rho^{*}\left(\pi_{1}^{*} \mathcal{O}_{X}(\Delta-F) \otimes \pi_{1,3}^{*} I_{\mathcal{Z}_{n_{+}}}\right) \rightarrow 0
\end{aligned}
$$

Remark 2.9. Very similar results hold in the case where $-K_{X}$ is effective and nonzero (corresponding to certain elliptic ruled surfaces) or $K_{X}=0$ (corresponding to $K 3$ or abelian surfaces). For example, in the case of a $K 3$ surface $X$, the moduli space is smooth of dimension $-p-6$ away from the sheaves which are strictly semistable for every ample divisor (although there exist components consisting entirely of non-locally free sheaves for small values of $-p$ ). In this case however $h(\zeta)=-\zeta^{2} / 2-2$ and $N_{\zeta}+N_{-\zeta}+2 \ell_{\zeta}=$ $-p-6$, which is equal to the dimension $d$ of the moduli space instead of to $d-1$. For example, if $\ell_{\zeta}=0$, then $N_{\zeta}=N_{-\zeta}=d / 2$. In this case $E_{\zeta}^{0,0} \cong \mathbb{P}^{d / 2}$ is a maximal isotropic submanifold of the symplectic manifold $\mathfrak{M}_{-}$. In other words, the natural holomorphic 2-form $\omega$ on $\mathfrak{M}_{-}$vanishes on $E_{\zeta}^{0,0}$ and identifies the normal bundle of $E_{\zeta}^{0,0}$ in $\mathfrak{M}_{\text {- with the cotangent }}$ bundle of $E_{\zeta}^{0,0}$.

\section{Flips of moduli spaces.}

In this section, we begin by assuming again that $X$ is an arbitrary algebraic surface. Let $\zeta=\zeta_{1}, \ldots, \zeta_{n}$ be the positive rational multiples of $\zeta$ such that $\zeta_{i}$ is an integral class also defining the wall $W^{\zeta}$. Our goal in this section is to deal with the problem that there is only a rational map in general from $E_{\zeta_{i}}^{n_{-}, n_{+}}$to $\mathfrak{M}_{-}$. We shall do so by finding a sequence of spaces between $\mathfrak{M}_{-}$and $\mathfrak{M}_{+}$, each one given by blowing up and down the previous one, such that for an appropriate member of the sequence the rational map $E_{\zeta_{i}}^{n_{-}, n_{+}} \rightarrow \mathfrak{M}_{-}$becomes a morphism (and a smooth embedding in the case of rational surfaces). Throughout the rest of this paper, $L_{0}$ shall denote any ample divisor contained in the interior of the intersection of $W^{\zeta}$ and the closures of $\mathcal{C}_{ \pm}$. Recall that we have defined universal semistability after the proof of (2.1). 
Definition 3.1. Let $k$ be an integer. A rank two torsion free sheaf $V$ with $c_{1}(V)=\Delta$ and $\Delta^{2}-4 c_{2}(V)=p$ is $\left(L_{0}, \zeta, k\right)$-semistable if $V$ is Mumford $L_{0}$-semistable and if it is strictly Mumford semistable, then either it is universally semistable or, for all divisors $F$ such that $2 F-\Delta \equiv \zeta$, we have the following:

(i) If there exists an exact sequence

$$
0 \rightarrow \mathcal{O}_{X}(F) \otimes I_{Z_{1}} \rightarrow V \rightarrow \mathcal{O}_{X}(\Delta-F) \otimes I_{Z_{2}} \rightarrow 0,
$$

then $\ell\left(Z_{2}\right) \leq k$ and thus $\ell\left(Z_{1}\right) \geq \ell_{\zeta}-k$.

(ii) If there exists an exact sequence

$$
0 \rightarrow \mathcal{O}_{X}(\Delta-F) \otimes I_{Z_{1}} \rightarrow V \rightarrow \mathcal{O}_{X}(F) \otimes I_{Z_{2}} \rightarrow 0,
$$

then $\ell\left(Z_{1}\right) \geq k+1$ and thus $\ell\left(Z_{2}\right) \leq \ell_{\zeta}-k-1$.

Likewise, setting $\boldsymbol{\zeta}=\left(\zeta_{1}, \ldots, \zeta_{n}\right)$ and $\mathbf{k}=\left(k_{1}, \ldots, k_{n}\right)$, we say that $V$ is $\left(L_{0}, \zeta, \mathbf{k}\right)$-semistable if $V$ is $\left(L_{0}, \zeta_{i}, k_{i}\right)$-semistable for every $i$. Let $\mathfrak{M}_{0}^{(\zeta, \mathbf{k})}$ denote the set of isomorphism classes of $\left(L_{0}, \boldsymbol{\zeta}, \mathbf{k}\right)$-semistable rank two sheaves $V$ with $c_{1}(V)=\Delta$ and $\Delta^{2}-4 c_{2}(V)=p$.

Next we give some easy properties of $\left(L_{0}, \boldsymbol{\zeta}, \mathbf{k}\right)$-semistability.

Lemma 3.2. (i) If $k_{i} \geq \ell_{\zeta_{i}}$ for all $i$, and $V$ is not universally semistable, then $V$ is $\left(L_{0}, \boldsymbol{\zeta}, \mathbf{k}\right)$-semistable if and only if it is $L_{-}$-stable. Likewise if $k_{i} \leq-1$ for all $i$ and $V$ is not universally semistable, then $V$ is $\left(L_{0}, \zeta, \mathbf{k}\right)$-semistable if and only if it is $L_{+}$-stable.

(ii) If $k_{i} \geq \ell_{\zeta_{i}}$ for all $i$, then $\mathfrak{M}_{0}^{(\zeta, \mathbf{k})}=\mathfrak{M}_{-}$. Likewise if $k_{i} \leq-1$ for all $i$, then $\mathfrak{M}_{0}^{(\zeta, \mathbf{k})}=\mathfrak{M}_{+}$.

(iii) For $n_{2}>k_{i}, \mathfrak{M}_{0}^{(\zeta, \mathbf{k})} \cap E_{\zeta_{i}}^{n_{1}, n_{2}}=\emptyset$.

(iv) There is an injection $E_{\zeta_{i}}^{\ell_{\zeta_{i}}-k_{i}, k_{i}} \rightarrow \mathfrak{M}_{0}^{(\zeta, k)}$. Likewise there is an injection $E_{-\zeta_{i}}^{k_{i}+1, \ell_{\zeta_{i}}-k_{i}-1} \rightarrow \mathfrak{M}_{0}^{(\boldsymbol{\zeta}, \mathbf{k})}$. Finally, the images of $E_{\zeta_{i}}^{\ell_{\zeta_{i}}-k_{i}, k_{i}}$ and $E_{\zeta_{j}}^{\ell_{\zeta_{j}}-k_{j}, k_{j}}$ are disjoint if $i \neq j$.

Proof. If $k_{i} \geq \ell_{\zeta_{i}}$ for all $i$, then the condition that $\ell\left(Z_{2}\right) \leq \ell_{\zeta_{i}}$ and $\ell\left(Z_{1}\right) \geq 0$ are trivially always satisfied and the conditions $\ell\left(Z_{2}\right) \leq-1$ and $\ell\left(Z_{1}\right) \geq$ 
$\ell_{\zeta_{i}}+1$ are vacuous. A similar argument handles the case $k_{i} \leq-1$ for all $i$. It is easy to see that this implies (i). Statement (ii) follows from (i), and (iii) follows from the definitions. As for (iv), let $V \in E_{\zeta_{i}}^{\ell_{\zeta_{i}}-k_{i}, k_{i}}$. To decide if $V$ is in $\mathfrak{M}_{0}^{(\zeta, \mathbf{k})}$, we look for potentially destabilizing subsheaves with torsion free quotient. Similar arguments as in [31] show that the only potentially destabilizing subsheaves with torsion free quotient must be either $\mathcal{O}_{X}(F) \otimes I_{Z_{1}}$ or $\mathcal{O}_{X}(\Delta-F) \otimes I_{Z}$. By hypothesis, there is a unique subsheaf of $V$ of the form $\mathcal{O}_{X}(F) \otimes I_{Z_{1}}$, and it is not destabilizing. If there is a subsheaf of the form $\mathcal{O}_{X}(\Delta-F) \otimes I_{Z}$ with torsion free quotient, then by Lemma 2.2 we have $\ell(Z)>\ell\left(Z_{2}\right)=k_{i}$ and so $\ell(Z) \geq k_{i}+1$. Hence such a subsheaf is also not destabilizing. Thus by Definition $3.1 \mathrm{~V}$ is $\left(L_{0}, \boldsymbol{\zeta}, \mathbf{k}\right)$-semistable. The fact that the map $E_{\zeta_{i}}^{\ell_{\zeta_{i}}-k_{i}, k_{i}} \rightarrow \mathfrak{M}_{0}^{(\zeta, \mathbf{k})}$ is one-to-one and that $E_{\zeta_{i}}^{\ell_{\zeta_{i}}-k_{i}, k_{i}}$ and $E_{\zeta_{j}}^{\ell_{\zeta_{j}}-k_{j}, k_{j}}$ are disjoint if $i \neq j$ also follow from similar arguments in [31]. The statement about $E_{-\zeta_{i}}^{k_{i}+1, \ell_{\zeta_{i}}-k_{i}-1}$ is similar.

Next suppose that we are given two integral vectors $\mathbf{k}$ and $\mathbf{k}^{\prime}$ and a subset $I$ of $\{1 \ldots, n\}$ such that $k_{i}^{\prime}=k_{i}$ if $i \notin I$ and $k_{i}^{\prime}=k_{i}-1$ if $i \in I$. We investigate the change as we pass from $\mathfrak{M}_{0}^{(\zeta, \mathbf{k})}$ to $\mathfrak{M}_{0}^{\left(\boldsymbol{\zeta}, \mathbf{k}^{\prime}\right)}$.

Lemma 3.3. The set of sheaves $V$ in $\mathfrak{M}_{0}^{(\zeta, \mathbf{k})}$ which are not $\left(L_{0}, \boldsymbol{\zeta}, \mathbf{k}^{\prime}\right)$ semistable is exactly the image of $\bigcup_{i \in I} E_{\zeta_{i}}^{\ell_{\zeta_{i}}-k_{i}, k_{i}}$. Likewise the set of $V \in$ $\mathfrak{M}_{0}^{\left(\zeta, \mathbf{k}^{\prime}\right)}$ which are not $\left(L_{0}, \boldsymbol{\zeta}, \mathbf{k}\right)$-semistable is exactly the image of $\bigcup_{i \in I} E_{-\zeta_{i}}^{k_{i}, \ell_{\zeta_{i}}-k_{i}}$

Proof. If $V$ is $\left(L_{0}, \boldsymbol{\zeta}, \mathbf{k}\right)$-semistable but not $\left(L_{0}, \boldsymbol{\zeta}, \mathbf{k}^{\prime}\right)$-semistable, then $V$ must be Mumford strictly $L_{0}$-semistable. Suppose that the $\left(L_{0}, \boldsymbol{\zeta}, \mathbf{k}^{\prime}\right)$ destabilizing subsheaf is of the form $\mathcal{O}_{X}(F) \otimes I_{Z_{1}}$, where $F$ corresponds to $\zeta_{i}$ for some $i \in I$. Then $\ell\left(Z_{2}\right) \leq k_{i}$ (since $V \in \mathfrak{M}_{0}^{(\boldsymbol{\zeta}, \mathbf{k})}$ ) but $\ell\left(Z_{2}\right) \geq k_{i}$ (since the subsheaf is $\left(L_{0}, \boldsymbol{\zeta}, \mathbf{k}^{\prime}\right)$-destabilizing, for $\left.k_{i}^{\prime}=k_{i}-1\right)$ so that $\ell\left(Z_{2}\right)=k_{i}$. Thus $V \in E_{\zeta}^{\ell_{\zeta_{i}}-k_{i}, k_{i}}$. The other possibility is that the destabilizing subsheaf is of the form $\mathcal{O}_{X}(\Delta-F) \otimes I_{Z_{1}}$. Here we need $\ell\left(Z_{1}\right) \geq k_{i}+1$ but $\ell\left(Z_{1}\right)<k_{i}$ and there are no such sheaves. The statement about $\mathfrak{M}_{0}^{\left(\zeta, \mathbf{k}^{\prime}\right)}$ follows by symmetry.

We shall now describe a sequence of actual moduli spaces $\mathfrak{M}_{0}^{(\boldsymbol{\zeta}, \mathbf{k})}$ for which the integral vector $\mathbf{k}$ change in the way described before the statement 
of (3.3).

Definition 3.4. Suppose that $\zeta_{i}=r_{i} \zeta_{1}$, where $r_{i}$ is a positive rational number. Given $t \in \mathbb{Q}$, let $t_{i}=r_{i} t$, so that $t_{1}=t$. Suppose that $\frac{\ell_{\zeta_{i}}+t_{i}}{2}$ is not an integer for any $i$. In this case, define

$$
k_{i}(t)=\left[\frac{\ell_{\zeta_{i}}+t_{i}}{2}\right]
$$

where $[x]$ is the greatest integer function, and define $\mathbf{k}(t)$ to be the vector formed by the $k_{i}(t)$. A rational number $t$ is $\zeta_{i}$-critical if $\frac{\ell_{\zeta_{i}}+t_{i}}{2} \in \mathbb{Z}$ and $-1 \leq \frac{\ell_{\zeta_{i}}+t_{i}}{2} \leq \ell_{\zeta_{i}}$. We shall also say that $t_{i}$ is $\zeta_{i}$-critical. Finally $t$ is $\zeta$-critical if it is $\zeta_{i}$-critical for some $i$. Note that there are only finitely many such $t$.

Given $t \in \mathbb{Q}$, let $I(t)=\left\{i: t\right.$ is $\zeta_{i}$-critical $\}$. Suppose that $\varepsilon$ is chosen so that, for every $i$, either there is no $\zeta_{i}$-critical rational number in $\left[t_{i}-\right.$ $\left.r_{i} \varepsilon, t_{i}+r_{i} \varepsilon\right]$ or $t_{i}$ is the unique $\zeta_{i}$-critical rational number in $\left[t_{i}-r_{i} \varepsilon, t_{i}+r_{i} \varepsilon\right]$. Equivalently either there is no $\zeta$-critical number in $[t-\varepsilon, t+\varepsilon]$ or $t$ is the unique $\zeta$-critical number in $[t-\varepsilon, t+\varepsilon]$. Then we clearly have:

$$
k_{i}(t-\varepsilon)= \begin{cases}k_{i}(t+\varepsilon), & \text { if } i \notin I(t) \\ k_{i}(t+\varepsilon)-1, & \text { if } i \in I(t) .\end{cases}
$$

In particular if there is no $\zeta$-critical number in $[t-\varepsilon, t+\varepsilon]$, so that $I(t)=\emptyset$, then $k_{i}(t-\varepsilon)=k_{i}(t+\varepsilon)$ for every $i$. Further note that if $t \gg 0$, then $k_{i}(t)>\ell_{\zeta_{i}}$ for every $i$, and if $t \ll 0$, then $k_{i}(t)<-1$ for every $i$.

We then have the following theorem, whose proof will be given in the next section:

Theorem 3.5. For all $t \in \mathbb{Q}$ which are not $\zeta$-critical, there exists a natural structure of a projective scheme on $\mathfrak{M}_{0}^{(\zeta, \mathbf{k}(t))}$ for which it is a coarse moduli space.

The proof of (3.5) will also show that $\mathfrak{M}_{0}^{(\zeta, \mathbf{k}(t))}$ has the usual properties of a coarse moduli space: all sheaves corresponding to points of $\mathfrak{M}_{0}^{(\zeta, \mathbf{k}(t))}$ will turn out to be simple (as they will turn out to be stable for an appropriate notion of stability), a classical or formal neighborhood of a point of $\mathfrak{M}_{0}^{(\boldsymbol{\zeta}, \mathbf{k}(t))}$ may be identified with the universal deformation space of the corresponding 
sheaf, and there exists a universal sheaf locally in the classical or étale topology around every point of $\mathfrak{M}_{0}^{(\zeta, \mathbf{k}(t))}$.

For the rest of this section, we shall again restrict to the case where $X$ is a rational surface with $-K_{X}$ effective, unless otherwise noted. Let $\zeta=\zeta_{i}$ for some $i$ and let $\mathbf{k}=\mathbf{k}(t)$ for some $t$ which is not $\zeta$-critical. The first step is to make some infinitesimal calculations concerning the differential of the map $E_{\zeta}^{\ell_{\zeta}-k, k} \rightarrow \mathfrak{M}_{0}^{(\zeta, \mathbf{k})}$ and the normal bundle to its image.

Proposition 3.6. The map $E_{\zeta}^{\ell_{\zeta}-k, k} \rightarrow \mathfrak{M}_{0}^{(\zeta, \mathbf{k})}$ is an immersion. The normal bundle $\mathcal{N}_{\zeta}^{\ell_{\zeta}-k, k}$ to $E_{\zeta}^{\ell_{\zeta}-k, k}$ in $\mathfrak{M}_{0}^{(\zeta, \mathbf{k})}$ is exactly $\rho^{*} \mathcal{E}_{-\zeta}^{k, \ell_{\zeta}-k} \otimes \mathcal{O}_{E_{\zeta}^{\ell_{\zeta}-k, k}}(-1)$, in the notation of the previous section.

Proof. Since every sheaf in $\mathfrak{M}_{0}^{(\boldsymbol{\zeta}, \mathbf{k})}$ is actually stable and therefore simple (which was also proved in (2.2)) we may identify an analytic neighborhood of $V \in \mathfrak{M}_{0}^{(\zeta, \mathbf{k})}$ with the germ of the universal deformation space for $V$, i.e. with $\operatorname{Ext}^{1}(V, V)$. Let us now calculate the tangent space to $E_{\zeta}^{\ell_{\zeta}-k, k}$ at $V$ : suppose that $\xi \in \operatorname{Ext}^{1}\left(\mathcal{O}_{X}(\Delta-F) \otimes I_{Z_{2}}, \mathcal{O}_{X}(F) \otimes I_{Z_{1}}\right)=\operatorname{Ext}^{1}$ is a nonzero extension class corresponding to $V$, where $\ell\left(Z_{1}\right)=\ell_{\zeta}-k$ and $\ell\left(Z_{2}\right)=k$. Let $H_{\ell_{\zeta}-k}=\mathrm{Hilb}^{\ell_{\zeta}-k} X$ and $H_{k}=$ Hilb $^{k} X$. Then there is the following exact sequence for the tangent space to $E_{\zeta}^{\ell_{\zeta}-k, k}$ at $\xi$ :

$$
0 \rightarrow \operatorname{Ext}^{1} / \mathbb{C} \cdot \xi \rightarrow T_{\xi} E_{\zeta}^{\ell_{\zeta}-k, k} \rightarrow T_{Z_{1}} H_{\ell_{\zeta}-k} \oplus T_{Z_{2}} H_{k} \rightarrow 0 .
$$

Note further that the tangent space to $\operatorname{Hilb}^{n} X$ at $Z$ is equal to $\operatorname{Hom}\left(I_{Z}, \mathcal{O}_{Z}\right)$, which we may further canonically identify with $\operatorname{Ext}^{1}\left(I_{Z}, I_{Z}\right)$ since $X$ is rational and by a local calculation. We then have the following:

Proposition 3.7. For all nonzero $\xi \in \mathrm{Ext}^{1}$, the natural map from a neighborhood of $\xi$ in $E_{\zeta}^{\ell_{\zeta}-k, k}$ to $\mathfrak{M}_{0}^{(\zeta, \mathbf{k})}$ is an immersion at $\xi$. The image of $T_{\xi} E_{\zeta}^{\ell_{\zeta}-k, k}$ in $\operatorname{Ext}^{1}(V, V)$ is exactly the kernel of the natural map

$$
\operatorname{Ext}^{1}(V, V) \rightarrow \operatorname{Ext}^{1}\left(\mathcal{O}_{X}(F) \otimes I_{Z_{1}}, \mathcal{O}_{X}(\Delta-F) \otimes I_{Z_{2}}\right),
$$

and the normal space to $E_{\zeta}^{\ell_{\zeta}-k, k}$ at $\xi$ in $\mathfrak{M}_{0}^{(\zeta, \mathbf{k})}$ may be canonically identified with $\operatorname{Ext}^{1}\left(\mathcal{O}_{X}(F) \otimes I_{Z_{1}}, \mathcal{O}_{X}(\Delta-F) \otimes I_{Z_{2}}\right)$. 
Proof. Consider the natural map from $\operatorname{Ext}^{1}(V, V)$ to

$$
\operatorname{Ext}^{1}\left(\mathcal{O}_{X}(F) \otimes I_{Z_{1}}, \mathcal{O}_{X}(\Delta-F) \otimes I_{Z_{2}}\right) .
$$

We claim that this map is surjective and will describe its kernel in more detail. The map factors into two maps:

$$
\begin{gathered}
\operatorname{Ext}^{1}(V, V) \rightarrow \operatorname{Ext}^{1}\left(V, \mathcal{O}_{X}(\Delta-F) \otimes I_{Z_{2}}\right) \\
\operatorname{Ext}^{1}\left(V, \mathcal{O}_{X}(\Delta-F) \otimes I_{Z_{2}}\right) \rightarrow \operatorname{Ext}^{1}\left(\mathcal{O}_{X}(F) \otimes I_{Z_{1}}, \mathcal{O}_{X}(\Delta-F) \otimes I_{Z_{2}}\right) .
\end{gathered}
$$

The cokernel of the first map is contained in $\operatorname{Ext}^{2}\left(V, \mathcal{O}_{X}(F) \otimes I_{Z_{1}}\right)$. To see that this group is zero, apply Serre duality: it suffices to show that $\operatorname{Hom}\left(\mathcal{O}_{X}(F) \otimes I_{Z_{1}}, V \otimes K_{X}\right)=0$. From the defining exact sequence for $V$, we have an exact sequence

$$
\begin{aligned}
& \rightarrow \operatorname{Hom}\left(\mathcal{O}_{X}(F) \otimes I_{Z_{1}}, \mathcal{O}_{X}(F) \otimes I_{Z_{1}} \otimes K_{X}\right) \\
& \rightarrow \operatorname{Hom}\left(\mathcal{O}_{X}(F) \otimes I_{Z_{1}}, V \otimes K_{X}\right) \rightarrow \operatorname{Hom}\left(\mathcal{O}_{X}(F) \otimes I_{Z_{1}}, \mathcal{O}_{X}(\Delta-F) \otimes I_{Z_{2}}\right) .
\end{aligned}
$$

The first term is just $H^{0}\left(K_{X}\right)=0$ and the third is contained in $H^{0}\left(\mathcal{O}_{X}(\Delta-\right.$ $\left.2 F) \otimes K_{X}\right)=0$. Thus $\operatorname{Hom}\left(\mathcal{O}_{X}(F) \otimes I_{Z_{1}}, V \otimes K_{X}\right)=0$. The vanishing of the cokernel of the second map, namely $\operatorname{Ext}^{2}\left(\mathcal{O}_{X}(\Delta-F) \otimes I_{Z_{2}}, \mathcal{O}_{X}(\Delta-F) \otimes I_{Z_{2}}\right)$, is similar. Thus $\operatorname{Ext}^{1}(V, V) \rightarrow \operatorname{Ext}^{1}\left(\mathcal{O}_{X}(F) \otimes I_{Z_{1}}, \mathcal{O}_{X}(\Delta-F) \otimes I_{Z_{2}}\right)$ is onto. If $K$ is the kernel, then arguments as above show that there is an exact sequence

$$
\begin{aligned}
0 \rightarrow \operatorname{Ext}^{1}\left(V, \mathcal{O}_{X}(F)\right. & \left.\otimes I_{Z_{1}}\right) \rightarrow K \rightarrow \\
& \rightarrow \operatorname{Ext}^{1}\left(\mathcal{O}_{X}(\Delta-F) \otimes I_{Z_{2}}, \mathcal{O}_{X}(\Delta-F) \otimes I_{Z_{2}}\right) \rightarrow 0 .
\end{aligned}
$$

Here $\operatorname{Ext}^{1}\left(\mathcal{O}_{X}(\Delta-F) \otimes I_{Z_{2}}, \mathcal{O}_{X}(\Delta-F) \otimes I_{Z_{2}}\right)=\operatorname{Ext}^{1}\left(I_{Z_{2}}, I_{Z_{2}}\right)$ is the tangent space to $H_{k}$. Moreover, there is an exact sequence

$$
\begin{gathered}
\quad \operatorname{Hom}\left(\mathcal{O}_{X}(F) \otimes I_{Z_{1}}, \mathcal{O}_{X}(F) \otimes I_{Z_{1}}\right) \rightarrow \\
\rightarrow \operatorname{Ext}^{1}\left(\mathcal{O}_{X}(\Delta-F) \otimes I_{Z_{2}}, \mathcal{O}_{X}(F) \otimes I_{Z_{1}}\right) \rightarrow \\
\quad \rightarrow \operatorname{Ext}^{1}\left(V, \mathcal{O}_{X}(F) \otimes I_{Z_{1}}\right) \rightarrow \\
\rightarrow \operatorname{Ext}^{1}\left(\mathcal{O}_{X}(F) \otimes I_{Z_{1}}, \mathcal{O}_{X}(F) \otimes I_{Z_{1}}\right) \rightarrow 0 .
\end{gathered}
$$

The last term is $\operatorname{Ext}^{1}\left(I_{Z_{1}}, I_{Z_{1}}\right)$ which is the tangent space to $H_{\ell_{\zeta}-k}$ at $Z_{1}$, and the first two terms combine to give $\operatorname{Ext}^{1} / \mathbb{C} \cdot \xi$. Thus the kernel $K$ looks very much like the tangent space to $E_{\zeta}^{\ell_{\zeta}-k, k}$ at $\xi$ and both spaces have the same dimension. 
Let us describe the tangent space to $E_{\zeta}^{\ell_{\zeta}-k, k}$ at $\xi$ and the differential of the map $E_{\zeta}^{\ell_{\zeta}-k, k}$ to $\mathfrak{M}_{0}^{(\boldsymbol{\zeta}, \mathbf{k})}$ in more intrinsic terms. It is easy to see that a Spec $\mathbb{C}[\epsilon]$-valued point of $E_{\zeta}^{\ell_{\zeta}-k, k}$ which restricts to $\xi$ defines two codimension two subschemes $\mathcal{Z}_{1} \subseteq X \times \operatorname{Spec} \mathbb{C}[\epsilon], \mathcal{Z}_{2} \subseteq X \times \operatorname{Spec} \mathbb{C}[\epsilon]$, flat over Spec $\mathbb{C}[\epsilon]$, restricting to $Z_{i}$ over $X$, and an extension $\mathcal{V}$ over $X \times \operatorname{Spec} \mathbb{C}[\epsilon]$ of the form

$$
0 \rightarrow \pi_{1}^{*} \mathcal{O}_{X}(F) \otimes I_{\mathcal{Z}_{1}} \otimes \rightarrow \mathcal{V} \rightarrow \pi_{1}^{*} \mathcal{O}_{X}(\Delta-F) \otimes I_{\mathcal{Z}_{2}} \rightarrow 0 .
$$

Conversely such a choice of $\mathcal{Z}_{1}, \mathcal{Z}_{2}$ and $\mathcal{V}$ define a Spec $\mathbb{C}[\epsilon]$-valued point of $E_{\zeta}^{\ell_{\zeta}-k, k}$. Thus there is a commutative diagram with exact rows and columns:

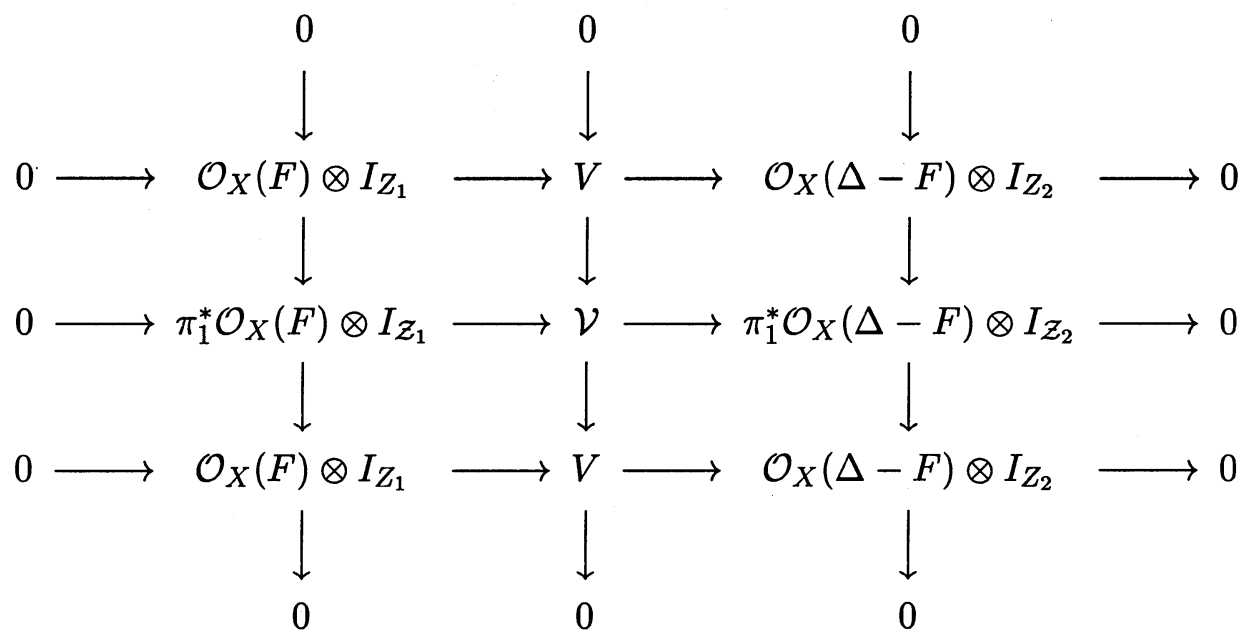

Here the extension $\mathcal{V}$ of $V$ by $V$, viewed as a point of $\operatorname{Ext}^{1}(V, V)$, corresponds to the Kodaira-Spencer map of the deformation $\mathcal{V}$ of $V$. Likewise the left and right hand columns give classes in $\operatorname{Ext}^{1}\left(I_{Z_{1}}, I_{Z_{1}}\right)$ and $\operatorname{Ext}^{1}\left(I_{Z_{2}}, I_{Z_{2}}\right)$ corresponding to $\mathcal{Z}_{1}$ and $\mathcal{Z}_{2}$. A straightforward diagram chase shows that if $\mathcal{V}$ fits into this commutative diagram then the image of the extension class $\xi \in \operatorname{Ext}^{1}(V, V)$ corresponding to $\mathcal{V}$ in $\operatorname{Ext}^{1}\left(\mathcal{O}_{X}(F) \otimes I_{Z_{1}}, \mathcal{O}_{X}(\Delta-F) \otimes I_{Z_{2}}\right)$ is zero. To see the converse, that every element in the kernel $K$ of the map $\operatorname{Ext}^{1}(V, V) \rightarrow \operatorname{Ext}^{1}\left(\mathcal{O}_{X}(F) \otimes I_{Z_{1}}, \mathcal{O}_{X}(\Delta-F) \otimes I_{Z_{2}}\right)$ is the image of a tangent vector to $E_{\zeta}^{\ell_{\zeta}-k, k}$ at $\xi$, use the arguments above which show that there is a surjection from $K$ to

$$
\operatorname{Ext}^{1}\left(\mathcal{O}_{X}(\Delta-F) \otimes I_{Z_{2}}, \mathcal{O}_{X}(\Delta-F) \otimes I_{Z_{2}}\right)=\operatorname{Ext}^{1}\left(I_{Z_{2}}, I_{Z_{2}}\right) .
$$

Thus there is an induced extension of $\mathcal{O}_{X}(\Delta-F) \otimes I_{Z_{2}}$ by $\mathcal{O}_{X}(\Delta-F) \otimes I_{Z_{2}}$, necessarily of the form $\mathcal{O}_{X}(\Delta-F) \otimes I_{\mathcal{Z}_{2}}$, and a map from $\mathcal{V}$ to $\mathcal{O}_{X}(\Delta-F) \otimes$ 
$I_{\mathcal{Z}_{2}}$, necessarily a surjection. The kernel of this surjection then defines an extension $\mathcal{O}_{X}(F) \otimes I_{\mathcal{Z}_{1}}$ of $\mathcal{O}_{X}(F) \otimes I_{Z_{1}}$ by $\mathcal{O}_{X}(F) \otimes I_{Z_{1}}$. It follows that $K$ is in the image of the tangent space to $E_{\zeta}^{\ell_{\zeta}-k, k}$ at $\xi$. By counting dimensions the map on tangent spaces from $T_{\xi} E_{\zeta}^{\ell_{\zeta}-k, k}$ to $\operatorname{Ext}^{1}(V, V)$ is injective, showing that the map from $E_{\zeta}^{\ell_{\zeta}-k, k}$ to $\mathfrak{M}_{0}^{(\zeta, \mathbf{k})}$ is an immersion and identifying the normal space at $\xi$.

Let us continue the proof of Proposition 3.6. To give a global description of the normal bundle to $E_{\zeta}^{\ell_{\zeta}-k, k}$ in $\mathfrak{M}_{0}^{(\zeta, \mathbf{k})}$, recall by standard deformation theory [10] that the pullback of the tangent bundle of $\mathfrak{M}_{0}^{(\zeta, \mathbf{k})}$ to $E_{\zeta}^{\ell_{\zeta}-k, k}$ is just $\operatorname{Ext}_{\pi_{2}}^{1}(\mathcal{V}, \mathcal{V})$, where $\mathcal{V}$ is the universal sheaf over $X \times E_{\zeta}^{\ell_{\zeta}-k, k}$ described in (2.8) and $\pi_{2}: X \times E_{\zeta}^{\ell_{\zeta}-k, k} \rightarrow E_{\zeta}^{\ell_{\zeta}-k, k}$ is the second projection. Moreover the calculations above globalize to show that the normal bundle is exactly

$$
\begin{gathered}
\operatorname{Ext}_{\pi_{2}}^{1}\left(\rho^{*}\left(\pi_{1}^{*} \mathcal{O}_{X}(F) \otimes \pi_{1,2}^{*} I_{\mathcal{Z}_{1}}\right) \otimes\right. \\
\left.\pi_{2}^{*} \mathcal{O}_{E_{\zeta}^{\zeta_{\zeta}}-k, k}(1), \rho^{*}\left(\pi_{1}^{*} \mathcal{O}_{X}(\Delta-F) \otimes \pi_{1,3}^{*} I_{\mathcal{Z}_{2}}\right)\right),
\end{gathered}
$$

where $\rho: X \times E_{\zeta}^{\ell_{\zeta}-k, k} \rightarrow X \times H_{\ell_{\zeta}-k} \times H_{k}$ is the natural projection. Using standard base change results and the projection formula, we see that this sheaf is equal to

$$
\rho^{*} E x t_{\pi_{2}}^{1}\left(\pi_{1}^{*} \mathcal{O}_{X}(F) \otimes \pi_{1,2}^{*} I_{\mathcal{Z}_{1}}, \pi_{1}^{*} \mathcal{O}_{X}(\Delta-F) \otimes \pi_{1,3}^{*} I_{\mathcal{Z}_{2}}\right) \otimes \mathcal{O}_{E_{\zeta}^{\ell^{\zeta}-k, k}}(-1),
$$

which is the same as $\rho^{*} \mathcal{E}_{-\zeta}^{k, \ell_{\zeta}-k} \otimes \mathcal{O}_{E_{\zeta}^{\ell_{\zeta}-k, k}}(-1)$.

Finally, to compare the moduli space $\mathfrak{M}_{0}^{(\zeta, \mathbf{k}(t+\varepsilon))}$ with $\mathfrak{M}_{0}^{(\zeta, \mathbf{k}(t-\varepsilon))}$, where $t$ is the unique $\boldsymbol{\zeta}$-critical point in $[t-\varepsilon, t+\varepsilon]$, we shall need the following result which is a straightforward generalization of (A.2) of [11].

Proposition 3.8. Let $X$ be a smooth projective scheme or compact complex manifold, and let $T$ be smooth. Suppose that $\mathcal{V}$ is a rank two reflexive sheaf over $X \times T$, flat over $T$. Let $D$ be a reduced divisor on $T$, not necessarily smooth and let $i: D \rightarrow T$ be the inclusion. Suppose that $L$ is a line bundle on $X$ and that $\mathcal{Z}$ is a codimension two subscheme of $X \times D$, flat over $D$. Suppose further that $\mathcal{V} \rightarrow i_{*} \pi_{1}^{*} L \otimes I_{\mathcal{Z}}$ is a surjection, and let $\mathcal{V}^{\prime}$ be its kernel:

$$
0 \rightarrow \mathcal{V}^{\prime} \rightarrow \mathcal{V} \rightarrow i_{*} \pi_{1}^{*} L \otimes I_{\mathcal{Z}} \rightarrow 0
$$


Then there is a line bundle $M$ on $X$ and a subscheme $\mathcal{Z}^{\prime}$ of $X \times D$ codimension at least two, flat over $D$, with the following properties:

(i) $\mathcal{V}^{\prime}$ is reflexive and flat over $T$.

(ii) There are exact sequences

$$
\begin{gathered}
0 \rightarrow \pi_{1}^{*} M \otimes I_{\mathcal{Z}^{\prime}} \rightarrow \mathcal{V} \mid D \rightarrow \pi_{1}^{*} L \otimes I_{\mathcal{Z}} \rightarrow 0 \\
0 \rightarrow \pi_{1}^{*} L \otimes I_{\mathcal{Z}} \otimes \mathcal{O}_{D}(-D) \rightarrow \mathcal{V}^{\prime} \mid D \rightarrow \pi_{1}^{*} M \otimes I_{\mathcal{Z}^{\prime}} \rightarrow 0
\end{gathered}
$$

which restrict for each $t \in D$ to give exact sequences

$$
\begin{aligned}
& 0 \rightarrow M \otimes I_{Z^{\prime}} \rightarrow V_{t} \rightarrow L \otimes I_{Z} \rightarrow 0 ; \\
& 0 \rightarrow L \otimes I_{Z} \rightarrow\left(V_{t}\right)^{\prime} \rightarrow M \otimes I_{Z^{\prime}} \rightarrow 0 .
\end{aligned}
$$

Here $Z$ is the subscheme of $X$ defined by $\mathcal{Z}$ for the slice $X \times\{t\}$ and $Z^{\prime}$ is likewise defined by $\mathcal{Z}^{\prime}$.

(iii) If $D$ is smooth, then the extension class corresponding to $\left(V_{t}\right)^{\prime}$ in $\operatorname{Ext}^{1}\left(M \otimes I_{W}, L \otimes I_{Z}\right)$ is defined by the image of the normal vector to $D$ at $t$ under the composition of the Kodaira-Spencer map from the tangent space of $T$ at $t$ to $\operatorname{Ext}^{1}\left(V_{t}, V_{t}\right)$, followed by the natural map $\operatorname{Ext}^{1}\left(V_{t}, V_{t}\right) \rightarrow \operatorname{Ext}^{1}\left(M \otimes I_{Z^{\prime}}, L \otimes I_{Z}\right)$.

Here $\mathcal{V}^{\prime}$ is called the elementary modification of $\mathcal{V}$ along $D$. This construction has the following symmetry: if we make the elementary modification of $\mathcal{V}^{\prime}$ along $D$ corresponding to the surjection $\mathcal{V}^{\prime} \rightarrow i_{*}\left(\pi_{1}^{*} M \otimes I_{\mathcal{Z}^{\prime}}\right)$, then the result is $\mathcal{V} \otimes \mathcal{O}_{X \times T}(-(X \times D))$.

Here is the typical way that we will apply the above: given $X$, let $M$ be a smooth manifold and $Y$ a submanifold of $M$. Let $T$ be the blowup of $M$ along $Y$ and let $D$ be the exceptional divisor. Let $\pi: T \rightarrow M$ be the natural map. Then, given $\xi \in D$, the image in the normal space to $\pi(\xi)$ of the normal direction at $\xi$ to $D$ under $\pi_{*}$ may be identified with the line in the normal space corresponding to $\xi$.

We can now state the main result as follows:

Theorem 3.9. Suppose that $t$ is the unique $\zeta$-critical point in $[t-\varepsilon, t+\varepsilon]$. If $h\left( \pm \zeta_{i}\right)+\ell_{ \pm \zeta_{i}} \neq 0$ for every $i$, then the rational map $\mathfrak{M}_{0}^{(\zeta, \mathbf{k}(t+\varepsilon))} \rightarrow$ $\mathfrak{M}_{0}^{(\zeta, \mathbf{k}(t-\varepsilon))}$ is obtained as follows. For every $i$, fixing $\zeta_{i}=\zeta$ and $k_{i}(t+\varepsilon)=$ $k$, blow up $E_{\zeta}^{\ell_{\zeta}-k, k}$ in $\mathfrak{M}_{0}^{(\zeta, \mathbf{k}(t+\varepsilon))}$. Then the exceptional divisor $D$ is a

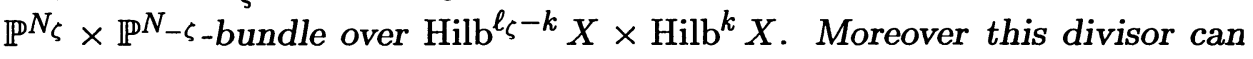


be contracted in two different ways. Contracting the $\mathbb{P}^{N_{-\zeta}}$ fibers for all possible $\zeta$ gives $\mathfrak{M}_{0}^{(\zeta, \mathbf{k}(t+\varepsilon))}$. Contracting the $\mathbb{P}^{N_{\zeta}}$ fibers for all possible $\zeta$ gives $\mathfrak{M}_{0}^{(\zeta, \mathbf{k}(t-\varepsilon))}$. Moreover the morphism from the blowup to $\mathfrak{M}_{0}^{(\zeta, \mathbf{k}(t-\varepsilon))}$ is induced by an elementary modification as in (3.8), and the image of the the component of the exceptional divisor which is the blowup of $E_{\zeta}^{\ell_{\zeta}-k, k}$ is $E_{-\zeta}^{k, \ell_{\zeta}-k}$. Finally the construction is symmetric.

Similar statements hold if $h\left( \pm \zeta_{i}\right)+\ell_{ \pm \zeta_{i}}=0$ for some $i$, where we must also add in or delete an extra component coming from $\pm \zeta_{i}$.

Proof. Begin by blowing up $E_{\zeta}^{\ell_{\zeta}-k, k}$ in $\mathfrak{M}_{0}^{(\zeta, \mathbf{k}(t+\varepsilon))}$ for all possible $\zeta$. For simplicity we shall just write down the argument in case there is only one $\zeta$; the general case is just additional notation. Let $\widetilde{\mathfrak{M}}_{0}^{(\zeta, k(t+\varepsilon))}$ denote the blowup and $D$ the exceptional divisor. Note that the normal bundle $\mathcal{N}_{\zeta}^{\ell_{\zeta}-k, k}$ to $E_{\zeta}^{\ell_{\zeta}-k, k}$ in $\mathfrak{M}_{0}^{(\zeta, \mathbf{k}(\mathbf{t}+\varepsilon))}$ is $\rho^{*} \mathcal{E}_{-\zeta}^{k, \ell_{\zeta}-k} \otimes \mathcal{O}_{E_{\zeta}^{\ell_{\zeta}-k, k}}(-1)$, where $\rho: E_{\zeta}^{\ell_{\zeta}-k, k} \rightarrow$ $\mathrm{Hilb}^{\ell_{\zeta}-k} X \times \mathrm{Hilb}^{k} X$ is the projection. In particular $\mathcal{N}_{\zeta}^{\ell_{\zeta}-k, k}$ restricts to each fiber $\mathbb{P}^{N_{\zeta}}$ to a bundle of the form $\left[\mathcal{O}_{\mathbb{P}^{N_{\zeta}}}^{N}\right] \otimes \mathcal{O}_{\mathbb{P}^{N_{\zeta}}}(-1)$, and an easy calculation using (2.7) shows that $N=N_{-\zeta}+1$. It follows that the fibers of the induced map from $D$ to $\mathrm{Hilb}^{\ell_{\zeta}-k} X \times$ Hilb $^{k} X$ are naturally $\mathbb{P}^{N_{\zeta}} \times \mathbb{P}^{N_{-\zeta}}$. Moreover it is easy to see that $\mathcal{O}(D) \mid \mathbb{P}^{N_{\zeta}}=\mathcal{O}_{\mathbb{P}^{N_{\zeta}}}(-1)$, using for example the fact that $\mathcal{O}(D) \mid \mathbb{P}^{N_{\zeta}} \times \mathbb{P}^{N_{-\zeta}}=\mathcal{O}(a,-1)$ for some $a$ and the fact that

$$
\mathcal{N}_{\zeta}^{\ell_{\zeta}-k, k} \mid \mathbb{P}^{N_{\zeta}}=R^{0} \pi_{1 *}\left[\mathcal{O}(-D) \mid \mathbb{P}^{N_{\zeta}} \times \mathbb{P}^{N_{-\zeta}}\right]=\left[\mathcal{O}_{\mathbb{P}^{N_{\zeta}}}^{N_{-\zeta}+1}\right] \otimes \mathcal{O}_{\mathbb{P}^{N_{\zeta}}}(-a) .
$$

For the rest of the argument, we assume that there exists a universal family on $X \times \mathfrak{M}_{0}^{(\zeta, k(t+\varepsilon))}$. Of course, such a family will only exist locally in the classical or étale topology, but this will suffice for the argument. Let $\mathcal{U}$ be the pullback of the universal family to $X \times \widetilde{\mathfrak{M}}_{0}^{(\zeta, \mathbf{k}(\mathbf{t}+\varepsilon))}$. Locally again we may assume that the restriction of $\mathcal{U}$ to $X \times D$ is the pullback of the universal extension $\mathcal{V}$ of (2.8):

$$
\begin{aligned}
0 & \rightarrow \rho^{*}\left(\pi_{1}^{*} \mathcal{O}_{X}(F) \otimes \pi_{1,2}^{*} I_{\mathcal{Z}_{n_{-}}}\right) \otimes \pi_{2}^{*} \mathcal{O}_{E_{\zeta}^{n_{-}, n_{+}}}(1) \\
& \rightarrow \mathcal{V} \rightarrow \rho^{*}\left(\pi_{1}^{*} \mathcal{O}_{X}(\Delta-F) \otimes \pi_{1,3}^{*} I_{\mathcal{Z}_{n_{+}}}\right) \rightarrow 0
\end{aligned}
$$

Now consider the effect of making an elementary transformation of $\mathcal{U}$ on $X \times \widetilde{\mathfrak{M}}_{0}^{(\boldsymbol{\zeta}, \mathbf{k}(\mathbf{t}+\varepsilon))}$ along the divisor $D$, using the morphism from $\mathcal{U}$ to the pullback of $\rho^{*}\left(\pi_{1}^{*} \mathcal{O}_{X}(\Delta-F) \otimes \pi_{1,3}^{*} I_{\mathcal{Z}_{k}}\right)$ given by considering the pullback 
of the universal extension. Applying (3.8) to the elementary transformation $\mathcal{U}^{\prime}$, we see that the fiber of $\mathcal{U}^{\prime}$ at a point of the fiber $\mathbb{P}^{N_{\zeta}} \times \mathbb{P}^{N_{-\zeta}}$ lying over a point $\left(Z_{1}, Z_{2}\right) \in \operatorname{Hilb}^{\ell_{\zeta}-k} X \times \operatorname{Hilb}^{k} X$ is given by a nonsplit extension of the form

$$
0 \rightarrow \mathcal{O}_{X}(\Delta-F) \otimes I_{Z_{2}} \rightarrow U \rightarrow \mathcal{O}_{X}(F) \otimes I_{Z_{1}} \rightarrow 0 .
$$

Moreover the extension class corresponding to $U$ is given by the projectivized normal vector in $\mathbb{P}^{N_{-\zeta}}$. Thus it is independent of the first factor $\mathbb{P}^{N_{\zeta}}$ and the set of all possible such classes is parametrized by the second factor $\mathbb{P}^{N_{-\zeta}}$. There is then an induced morphism from $\widetilde{\mathfrak{M}}_{0}^{(\zeta, \mathbf{k}(t+\varepsilon))}$ to $\mathfrak{M}_{0}^{(\zeta, \mathbf{k}(t-\varepsilon))}$ and clearly it has the effect of contracting $D$ along its first ruling and has the property that the image of $D$ is exactly $E_{-\zeta}^{k, \ell_{\zeta}-k}$. We leave the symmetry of the construction to the reader. This concludes the proof of (3.9).

Remark 3.10. In the $K 3$ or abelian case, the arguments of this section show that the rational map $\mathfrak{M}_{0}^{(\zeta, \mathbf{k}(\mathbf{t}+\varepsilon))} \rightarrow \mathfrak{M}_{0}^{(\zeta, \mathbf{k}(t-\varepsilon))}$ is a Mukai elementary transformation $[27,29]$.

We can also use (3.8) to analyze the rational map from $E_{\zeta}^{n_{-}, n_{+}}$to $\mathfrak{M}_{-}$, in the case where it is not a morphism. For simplicity we shall only consider the case of $E_{\zeta}^{1,0}$, i.e. $\ell_{\zeta}=1$. In this case $Z_{-}=p \in X$ and $I_{Z_{-}}=\mathfrak{m}_{p}$ is the maximal ideal sheaf of $p$. Moreover $\operatorname{Ext}^{1}\left(\mathcal{O}_{X}(\Delta-F), \mathcal{O}_{X}(F) \otimes \mathfrak{m}_{p}\right)=$ $H^{1}\left(\mathcal{O}_{X}(2 F-\Delta) \otimes \mathfrak{m}_{p}\right)$. There is an exact sequence

$$
0 \rightarrow H^{0}\left(\mathbb{C}_{p}\right) \rightarrow H^{1}\left(\mathcal{O}_{X}(2 F-\Delta) \otimes \mathfrak{m}_{p}\right) \rightarrow H^{1}\left(\mathcal{O}_{X}(2 F-\Delta)\right) \rightarrow 0 .
$$

Moreover, for $p$ fixed, the extensions $V$ corresponding to a split extension for $V^{\vee \vee}$ are exactly the kernel of the map from $H^{1}\left(\mathcal{O}_{X}(2 F-\Delta) \otimes \mathfrak{m}_{p}\right)$ to $H^{1}\left(\mathcal{O}_{X}(2 F-\Delta)\right)$, i.e. the image of $H^{0}\left(\mathbb{C}_{p}\right)$. The normal space is thus identified with $H^{1}\left(\mathcal{O}_{X}(2 F-\Delta)\right)$. Now if the extension for $V^{\vee \vee}$ is split, then there is a map $\mathcal{O}_{X}(\Delta-F) \otimes \mathfrak{m}_{p} \rightarrow V$ with quotient $\mathcal{O}_{X}(F)$. This way of realizing $V$ as an extension gives a surjection $\operatorname{Ext}^{1}(V, V) \rightarrow$ $\operatorname{Ext}^{1}\left(\mathcal{O}_{X}(\Delta-F) \otimes \mathfrak{m}_{p}, \mathcal{O}_{X}(F)\right)$, and we must look at the image of the normal space $H^{1}\left(\mathcal{O}_{X}(2 F-\Delta)\right)$ in this extension group. On the other hand, we have an exact sequence

$$
0 \rightarrow H^{1}\left(\mathcal{O}_{X}(2 F-\Delta)\right) \rightarrow \operatorname{Ext}^{1}\left(\mathcal{O}_{X}(\Delta-F) \otimes \mathfrak{m}_{p}, \mathcal{O}_{X}(F)\right) \rightarrow H^{0}\left(\mathbb{C}_{p}\right) \rightarrow 0
$$

coming from the long exact Ext sequence, and it is an easy diagram chase to see that the induced map $\operatorname{Ext}^{1}\left(\mathcal{O}_{X}(\Delta-F), \mathcal{O}_{X}(F) \otimes \mathfrak{m}_{p}\right) \rightarrow \operatorname{Ext}^{1}\left(\mathcal{O}_{X}(\Delta-\right.$ 
$\left.F) \otimes \mathfrak{m}_{p}, \mathcal{O}_{X}(F)\right)$ factors through the map $\operatorname{Ext}^{1}\left(\mathcal{O}_{X}(\Delta-F), \mathcal{O}_{X}(F) \otimes \mathfrak{m}_{p}\right) \rightarrow$ $H^{1}\left(\mathcal{O}_{X}(2 F-\Delta)\right)$ and that the image is exactly the natural subgroup $H^{1}\left(\mathcal{O}_{X}\right.$ $(2 F-\Delta))$ of $\operatorname{Ext}^{1}\left(\mathcal{O}_{X}(\Delta-F) \otimes \mathfrak{m}_{p}, \mathcal{O}_{X}(F)\right)$.

The above has the following geometric interpretation: the locus $U$ in $E_{\zeta}^{1,0}$ of $L_{-}$-unstable sheaves is in fact a section of $E_{\zeta}^{1,0}$. If we blow up this section and then make the elementary transformation, the result is exactly the set of elements of $E_{\zeta}^{0,1}$ corresponding to nonlocally free sheaves. This set is already a divisor in $E_{\zeta}^{0,1}$. There is thus a morphism from the blowup of $E_{\zeta}^{1,0}$ along $U$ to $\mathfrak{M}_{-}$which is an embedding into $\mathfrak{M}_{-}$. Its image $\left(E_{\zeta}^{1,0}\right)^{\prime}$ in $\mathfrak{M}_{-}$meets $E_{\zeta}^{0,1}$ exactly along the divisor in $E_{\zeta}^{0,1}$ of nonlocally free sheaves.

We can now give a picture of the birational map from $\mathfrak{M}_{-}$to $\mathfrak{M}_{+}$in this case. Begin with the subvariety $E_{\zeta}^{0,1}$ in $\mathfrak{M}_{-}$and blow it up. Let $D^{0,1}$ be the exceptional divisor, ruled in two different ways. As $E_{\zeta}^{0,1}$ meets $\left(E_{\zeta}^{1,0}\right)^{\prime}$ along a divisor, the proper transform of $\left(E_{\zeta}^{1,0}\right)^{\prime}$ in the blowup is again $\left(E_{\zeta}^{1,0}\right)^{\prime}$. Making the elementary modification along $D^{0,1}$, we then blow down $D^{0,1}$ to get a new moduli space. This moduli space then contains $E_{\zeta}^{1,0}$. At this point we can then blow up $E_{\zeta}^{1,0}$ and contract the new exceptional divisor $D^{1,0}$ to obtain $\mathfrak{M}_{+}$(a few extra details need to be checked here concerning the Kodaira-Spencer class). Note again the symmetry of the situation. In principle we could hope to carry through this analysis to the case where $\ell_{\zeta}>$ 1 as well, but we run into trouble with the birational geometry of Hilb ${ }^{n} X$. Somehow the construction of our auxiliary sequence of moduli spaces has eliminated the necessity for understanding this birational geometry in detail.

\section{Mixed stability and mixed moduli spaces.}

Our goal in this section is to give a proof of Theorem 3.5 (for an arbitrary algebraic surface $X$ ). By way of motivation for our construction, let us analyze Gieseker semistability more closely. In the notation of the last section, we suppose that $L_{0}$ is an ample line bundle lying on a unique wall $W$ of type $(w, p)$, and let $\zeta_{1}, \ldots, \zeta_{n}$ be the integral classes of type $(w, p)$ defining $W$. Let $V$ be an $L_{0}$-semistable rank two sheaf. Thus either $V$ is Mumford $L_{0}$-stable or it is Mumford strictly semistable. In the second case, let $\mathcal{O}_{X}(F) \otimes I_{Z_{1}}$ be a destabilizing subsheaf and suppose that there is an exact sequence

$$
0 \rightarrow \mathcal{O}_{X}(F) \otimes I_{Z_{1}} \rightarrow V \rightarrow \mathcal{O}_{X}(\Delta-F) \otimes I_{Z_{2}} \rightarrow 0
$$


Let $\zeta=2 F-\Delta$. We shall assume that $\zeta=\zeta_{i}$ for some $i$, or equivalently that $\zeta$ is not numerically equivalent to zero (i.e., $V$ is not universally semistable). By assumption $\mu_{L_{0}}(V) \geq \mu_{L_{0}}\left(\mathcal{O}_{X}(F) \otimes I_{Z_{1}}\right)$, and so $\chi(V) \geq 2 \chi\left(\mathcal{O}_{X}(F) \otimes\right.$ $\left.I_{Z_{1}}\right)$. Since $\chi(V)=\chi\left(\mathcal{O}_{X}(F) \otimes I_{Z_{1}}\right)+\chi\left(\mathcal{O}_{X}(\Delta-F) \otimes I_{Z_{2}}\right)$, we may rewrite this last condition as

$$
\chi\left(\mathcal{O}_{X}(\Delta-F) \otimes I_{Z_{2}}\right)-\chi\left(\mathcal{O}_{X}(F) \otimes I_{Z_{1}}\right) \geq 0 .
$$

Now from the exact sequence

$$
0 \rightarrow \mathcal{O}_{X}(F) \otimes I_{Z_{1}} \rightarrow \mathcal{O}_{X}(F) \rightarrow \mathcal{O}_{Z_{1}} \rightarrow 0,
$$

we see that $\chi\left(\mathcal{O}_{X}(F) \otimes I_{Z_{1}}\right)=\chi\left(\mathcal{O}_{X}(F)\right)-\ell\left(Z_{1}\right)$, and similarly $\chi\left(\mathcal{O}_{X}(\Delta-\right.$ $\left.F) \otimes I_{Z_{2}}\right)=\chi\left(\mathcal{O}_{X}(\Delta-F)\right)-\ell\left(Z_{2}\right)$. By Riemann-Roch,

$$
\begin{aligned}
\chi\left(\mathcal{O}_{X}(\Delta-F)\right)-\chi\left(\mathcal{O}_{X}(F)\right) & =\frac{1}{2}\left((\Delta-F)^{2}-(\Delta-F) \cdot K_{X}-F^{2}+F \cdot K_{X}\right) \\
& =\frac{1}{2}\left(\Delta^{2}-2 \Delta \cdot F+\zeta \cdot K_{X}\right) \\
& =\frac{1}{2} \zeta \cdot\left(K_{X}-\Delta\right)=t .
\end{aligned}
$$

Thus we have the following conditions on $Z_{1}$ and $Z_{2}$ :

$$
\begin{aligned}
& \ell\left(Z_{2}\right)-\ell\left(Z_{1}\right) \leq t ; \\
& \ell\left(Z_{2}\right)+\ell\left(Z_{1}\right)=\ell_{\zeta},
\end{aligned}
$$

and so $2 \ell\left(Z_{2}\right) \leq \ell_{\zeta}+t$. Setting $k=\left[\frac{\ell_{\zeta}+t}{2}\right]$, we have $\ell\left(Z_{2}\right) \leq k$. Applying a similar analysis to a subsheaf of the form $\mathcal{O}_{X}(\Delta-F) \otimes I_{Z_{1}}$ shows that, if there is such a subsheaf, with a torsion free quotient $\mathcal{O}_{X}(F) \otimes I_{Z_{2}}$, then

$$
\ell\left(Z_{2}\right) \leq \frac{\ell_{\zeta}-t}{2}=\ell_{\zeta}-\frac{\ell_{\zeta}+t}{2} .
$$

In particular, if $\frac{\ell_{\zeta}+t}{2}$ is not an integer, then this condition becomes $\ell\left(Z_{2}\right) \leq$ $\ell_{\zeta}-k-1$. Thus, provided $\frac{\ell_{\zeta}+t}{2}$ is not an integer for every $\zeta$ defining the wall $W$ (i.e. $t$ is not $\zeta$-critical for every $\zeta$ ), $V$ is $\left(L_{0}, \zeta, k\right)$-semistable for $k=\left[\frac{\ell_{\zeta}+t}{2}\right]$ and indeed $V$ is $\left(L_{0}, \zeta, \mathbf{k}\right)$-semistable, where $\mathbf{k}$ is defined in the obvious way. Conversely, assuming that $t$ is not $\zeta$-critical for every $\zeta$, 
$V$ is Gieseker $L_{0}$-semistable, indeed Gieseker $L_{0}$-stable, if it is $\left(L_{0}, \zeta, \mathbf{k}\right)$ semistable for $\mathbf{k}$ as above.

We would like to produce a similar condition where $t$ is allowed to be any rational number which is not $\zeta$-critical. One way to think of this problem is to consider the analogous problem where we replace $\Delta$ by $\Delta+2 \Xi$ and make the corresponding change in $c$, so that $\Delta$ and $p$ remain the same. This corresponds to twisting $V$ by $\mathcal{O}_{X}(\Xi)$; and $t$ is replaced by $t-\zeta \cdot \Xi$. In particular, we see that the notion of Gieseker stability is rather sensitive to twisting by a line bundle. Moreover if $W$ is defined by exactly one $\zeta$ such that there exists a divisor $\Xi$ with $\zeta \cdot \Xi=1$, for example if $\zeta$ is primitive and $p_{g}(X)=0$, it is easy to see that we can construct the appropriate moduli spaces as Gieseker moduli spaces corresponding to twists of $V$ by various multiples of $\Xi$. In general however we will need to consider a problem which is roughly analogous to allowing twists of $V$ by a $\mathbb{Q}$-divisor $\Xi$. This is the goal of the following definition of mixed stability:

Definition 4.1. Let $X$ be an algebraic surface and let $L_{0}$ be an ample line bundle on $X$. Fix line bundles $H_{1}$ and $H_{2}$ on $X$ and positive integers $a_{1}$ and $a_{2}$. For every torsion free sheaf $V$ on $X$ of rank $r$, define

$$
p_{V ; H_{1}, H_{2}, a_{1}, a_{2}}(n)=\frac{a_{1}}{r} \chi\left(V \otimes H_{1} \otimes L_{0}^{n}\right)+\frac{a_{2}}{r} \chi\left(V \otimes H_{2} \otimes L_{0}^{n}\right) .
$$

A torsion free sheaf $V$ is $\left(H_{1}, H_{2}, a_{1}, a_{2}\right) L_{0}$-stable if, for all subsheaves $W$ of $V$ with $0<\operatorname{rank} W<\operatorname{rank} V$ and for all $n \gg 0$,

$$
p_{V ; H_{1}, H_{2}, a_{1}, a_{2}}(n)>p_{W ; H_{1}, H_{2}, a_{1}, a_{2}}(n) .
$$

$\left(H_{1}, H_{2}, a_{1}, a_{2}\right) L_{0}$-semistable and unstable are defined similarly.

The usual arguments show the following:

Lemma 4.2. If $V$ is $\left(H_{1}, H_{2}, a_{1}, a_{2}\right) L_{0}$-stable, then it is simple.

In the case of rank two on a surface $X$ (which is the only case which shall concern us), $V$ is $\left(H_{1}, H_{2}, a_{1}, a_{2}\right) L_{0}$-stable if and only if, for all rank one subsheaves $W$, and for all $n \gg 0$, we have

$$
\begin{aligned}
& a_{1}\left(\chi\left(V \otimes H_{1} \otimes L_{0}^{n}\right)-2 \chi\left(W \otimes H_{1} \otimes L_{0}^{n}\right)\right)+ \\
& \quad a_{2}\left(\chi\left(V \otimes H_{2} \otimes L_{0}^{n}\right)-2 \chi\left(W \otimes H_{2} \otimes L_{0}^{n}\right)\right)>0 .
\end{aligned}
$$

In particular, if $V$ is $\left(H_{1}, H_{2}, a_{1}, a_{2}\right) L_{0}$-stable then either $V \otimes H_{1}$ or $V \otimes H_{2}$ is stable, and a similar statement holds for semistability. A short calculation 
shows that the coefficient of $n$ in the above expression (which is a degree two polynomial in $n)$ is $\left(a_{1}+a_{2}\right)\left(L_{0} \cdot\left(c_{1}(V)-2 F\right)\right)$ where $F=c_{1}(W)$ and that the constant term is

$$
\left(a_{1}+a_{2}\right)(\chi(V)-2 \chi(W))+a_{1} H_{1} \cdot\left(c_{1}(V)-2 F\right)+a_{2} H_{2} \cdot\left(c_{1}(V)-2 F\right) .
$$

Thus $V$ is $\left(H_{1}, H_{2}, a_{1}, a_{2}\right) L_{0}$-stable (resp. semistable) if and only if it is either Mumford $L_{0}$-stable or Mumford strictly semistable and the above constant term is positive (resp. nonnegative). It is easy to see, comparing this with the discussion at the beginning of this section, that formally this is the same as requiring that $V \otimes \Xi$ is (Gieseker) $L_{0}$-stable or semistable, where $\Xi$ is the $\mathbb{Q}$-divisor

$$
\frac{a_{1}}{a_{1}+a_{2}} H_{1}+\frac{a_{2}}{a_{1}+a_{2}} H_{2} .
$$

Thus for example taking $H_{2}=0$ and replacing $H_{1}$ by a positive integer multiple we see that we can take for $\Xi$ an arbitrary $\mathbb{Q}$-divisor.

Let us explicitly relate mixed stability to our previous notion of $\left(L_{0}, \boldsymbol{\zeta}, \mathbf{k}\right)$-semistability:

Lemma 4.3. Given $\Delta$ and $c$ and the corresponding $w$ and $p$, let $L_{0}$ be an ample divisor lying on a unique wall of type $(w, p)$ and let $V$ be a rank two torsion free sheaf with $c_{1}(V)=\Delta$ and $c_{2}(V)=c$. Let $\Xi$ be the $\mathbb{Q}$ divisor $\frac{a_{1}}{a_{1}+a_{2}} H_{1}+\frac{a_{2}}{a_{1}+a_{2}} H_{2}$ and suppose that the rational number $t_{i}=$ $\frac{1}{2} \zeta_{i} \cdot\left(K_{X}-\Delta\right)-\zeta_{i} \cdot \Xi$ is not $\zeta_{i}$-critical for every $\zeta_{i}$ of type $(w, p)$ defining $W$. Then, with $t=t_{1}, V$ is $\left(L_{0}, \boldsymbol{\zeta}, \mathbf{k}(t)\right)$-semistable if and only if it is $\left(H_{1}, H_{2}, a_{1}, a_{2}\right) L_{0}$-semistable if and only if it is $\left(H_{1}, H_{2}, a_{1}, a_{2}\right) L_{0}$-stable.

Proof. Using the additivity of the polynomials $p_{V ; H_{1}, H_{2}, a_{1}, a_{2}}$ over exact sequences, it is easy to check that $V$ is $\left(H_{1}, H_{2}, a_{1}, a_{2}\right) L_{0}$-semistable if and only if it is Mumford $L_{0}$-semistable, and for every Mumford destabilizing subsheaf of the form $\mathcal{O}_{X}(F) \otimes I_{Z_{1}}$, either $V$ is universally semistable or we have

$$
\chi(V)-2 \chi\left(\mathcal{O}_{X}(F) \otimes I_{Z_{1}}\right)-\zeta_{i} \cdot \Xi>0,
$$

where $\zeta_{i}=2 F-\Delta$. Using our calculations above, this works out to

$$
\ell\left(Z_{2}\right)-\ell\left(Z_{1}\right) \leq \frac{1}{2} \zeta_{i} \cdot\left(K_{X}-\Delta\right)-\zeta_{i} \cdot \Xi=t_{i} .
$$


Equivalently since $\ell\left(Z_{1}\right)+\ell\left(Z_{2}\right)=\ell_{\zeta_{i}}$, this becomes $\ell\left(Z_{2}\right) \leq\left[\frac{\ell_{\zeta_{i}}+t_{i}}{2}\right]$. Thus $V$ is $\left(H_{1}, H_{2}, a_{1}, a_{2}\right) L_{0}$-semistable if and only if it is $\left(L_{0}, \boldsymbol{\zeta}, \mathbf{k}(t)\right)$-semistable. Moreover, since $t$ is not $\zeta_{i}$-critical, the inequalities are automatically strict, so that $V$ is also $\left(H_{1}, H_{2}, a_{1}, a_{2}\right) L_{0}$-stable.

Now choosing a $\Xi_{0}$ such that $\zeta_{1} \cdot \Xi_{0} \neq 0$, every rational number $t$ is of the form $\frac{1}{2} \zeta_{1} \cdot\left(K_{X}-\Delta\right)-\zeta_{1} \cdot r \Xi_{0}$ for some rational number $r$. Thus Theorem 3.5 will follow from Lemma 4.3 and from the more general result below:

Theorem 4.4. Let $X$ be an algebraic surface $X$ and let $L_{0}$ be an ample line bundle on $X$. Given a divisor $\Delta$ and an integer $c$, line bundles $H_{1}$ and $H_{2}$ on $X$ and positive integers $a_{1}$ and $a_{2}$, suppose that every rank two torsion free sheaf $V$ with $c_{1}(V)=\Delta, c_{2}(V)=c$ which is $\left(H_{1}, H_{2}, a_{1}, a_{2}\right) L_{0}$-semistable is actually $\left(H_{1}, H_{2}, a_{1}, a_{2}\right) L_{0}$-stable. Then there exists a projective coarse moduli space $\mathfrak{M}_{L_{0}}\left(\Delta, c ; H_{1}, H_{2}, a_{1}, a_{2}\right)$ of isomorphism classes of rank two torsion free sheaves $V$ with $c_{1}(V)=\Delta, c_{2}(V)=c$, which are $\left(H_{1}, H_{2}, a_{1}, a_{2}\right)$ $L_{0}$-semistable.

Proof. The argument will follow the arguments in [13] as closely as possible, and we shall assume a familiarity with that paper.

Suppose that $V$ is $\left(H_{1}, H_{2}, a_{1}, a_{2}\right) L_{0}$-semistable. Then either $V \otimes H_{1}$ or $V \otimes H_{2}$ is $L_{0}$-semistable, and thus by [13], Lemma 1.3 the set of all such $V$ is bounded. We may thus choose an $n$ such that, for all $V$ which are $\left(H_{1}, H_{2}, a_{1}, a_{2}\right) L_{0}$-semistable, $V \otimes H_{i} \otimes L_{0}^{n}$ is generated by its global sections and has no higher cohomology, for $i=1,2$. Fix such an $n$ for the moment, and let $d_{i}=h^{0}\left(V \otimes H_{i} \otimes L_{0}^{n}\right)$. Then $d_{i}$ is independent of $V$ and $V$ is a quotient of $\left(H_{i}^{-1} \otimes L_{0}^{-n}\right)^{\oplus d_{i}}$. Let $Q_{i}$ be the open subset of the corresponding Quot scheme associated to $\left(H_{i}^{-1} \otimes L_{0}^{-n}\right)^{\oplus d_{i}}$ consisting of quotients which are rank two torsion free sheaves $V_{i}$ with $c_{1}\left(V_{i}\right)=\Delta$ and $c_{2}\left(V_{i}\right)=c$, and such that $V_{i} \otimes H_{i} \otimes L_{0}^{n}$ is generated by its global sections and has no higher cohomology. We will write a point of $Q_{i}$ as $V_{i}$, suppressing the surjection $\left(H_{i}^{-1} \otimes L_{0}^{-n}\right)^{\oplus d_{i}} \rightarrow V_{i}$. Inside $Q_{1} \times Q_{2}$, we have the closed subscheme $I_{0}$ consisting of quotients $V_{1}$ and $V_{2}$ such that $\operatorname{dim} \operatorname{Hom}\left(V_{1}, V_{2}\right) \geq 1$. There is also the open subvariety $I_{0}^{\prime}$ of $I_{0}$ consisting of $\left(V_{1}, V_{2}\right)$ with $\operatorname{dim} \operatorname{Hom}\left(V_{1}, V_{2}\right)=1$. Using the universal sheaves $\mathcal{U}_{i}$ over $X \times Q_{i}$, we can construct a $\mathbb{C}^{*}$ bundle $I$ over $I_{0}^{\prime}$ whose points are $\left(V_{1}, V_{2}, \varphi\right)$, where $\varphi: V_{1} \rightarrow V_{2}$ is a nonzero homomorphism, unique up to scalars.

For $i=1,2$, let $E_{i}$ be a fixed vector space of dimension equal to $d_{i}=$ $h^{0}\left(V \otimes H_{i} \otimes L_{0}^{n}\right)$. Fix once and for all an isomorphism $\left(H_{i}^{-1} \otimes L_{0}^{-n}\right)^{\oplus d_{i}} \cong$ 
$\left(H_{i}^{-1} \otimes L_{0}^{-n}\right) \otimes E_{i}$. A surjection $\left(H_{i}^{-1} \otimes L_{0}^{-n}\right)^{\oplus d_{i}} \rightarrow V_{i}$ then gives a map $E_{i} \rightarrow$ $H^{0}\left(V_{i} \otimes H_{i} \otimes L_{0}^{n}\right)$ and via such a surjection a basis $v_{1}, \ldots, v_{d_{1}}$ of $E_{1}$ gives $d_{1}$ sections of $V_{1} \otimes H_{1} \otimes L_{0}^{n}$ and similarly for a basis $w_{1}, \ldots, w_{d_{2}}$ of $E_{2}$. Moreover $G L\left(d_{i}\right)$ acts on $\left(H_{i}^{-1} \otimes L_{0}^{-n}\right)^{\oplus d_{i}}$ and on $Q_{i}$. By the universal property of the Quot scheme, this action extends to a $G L\left(d_{i}\right)$-linearization of the universal sheaf $\mathcal{U}_{i}$ over $X \times Q_{i}$. Thus there is a right action of $G L\left(d_{1}\right) \times G L\left(d_{2}\right)$ on $I$, and it is easy to see that the elements ( $\lambda \mathrm{Id}, \lambda \mathrm{Id})$ act trivially. Let $F_{i}$ be the fixed vector space $H^{0}\left(\Delta \otimes H_{i}^{2} \otimes L_{0}^{2 n}\right)$, and $F$ the fixed vector space $H^{0}\left(\Delta \otimes H_{1} \otimes H_{2} \otimes L_{0}^{2 n}\right)$. Let

$$
U=\operatorname{Hom}\left(\bigwedge^{2} E_{1}, F_{1}\right) \oplus \operatorname{Hom}\left(\bigwedge^{2} E_{2}, F_{2}\right) \oplus \operatorname{Hom}\left(E_{1} \otimes E_{2}, F\right) .
$$

(The factor $\operatorname{Hom}\left(E_{1} \otimes E_{2}, F\right)$ is there to make sure that the destabilizing subsheaves for $V \otimes H_{1}$ and $V \otimes H_{2}$ are in fact the same.) Note that $G L\left(d_{1}\right) \times$ $G L\left(d_{2}\right)$ operates on the right on $U$ and $\mathbb{P} U$. For example, the pair ( $\left.\lambda \mathrm{Id}, \mu \mathrm{Id}\right)$ acts on the triple $\left(T_{1}, T_{2}, T\right) \in U$ via $\left(T_{1}, T_{2}, T\right) \mapsto\left(\lambda^{2} T_{1}, \mu^{2} T_{2}, \lambda \mu T\right)$. Thus $\left(A_{1}, A_{2}\right)$ acts trivially on $\mathbb{P} U$ if and only if $\left(A_{1}, A_{2}\right)=(\lambda \mathrm{Id}, \lambda \mathrm{Id})$. Given a quintuple $\underline{V}=\left(V_{1}, V_{2}, \psi_{1}, \psi_{2}, \varphi\right)$, where $V_{i} \in Q_{i}, \psi_{i}: E_{i} \rightarrow H^{0}\left(V_{i} \otimes H_{i} \otimes L_{0}^{n}\right)$ is an isomorphism, and $\varphi: V_{1} \rightarrow V_{2}$ is a nonzero map, we will define a point $\left(T_{1}(\underline{V}), T_{2}(\underline{V}), T(\underline{V})\right) \in \mathbb{P} U$. To do so, fix an isomorphism $\alpha_{2}$ : $\operatorname{det} V_{2} \rightarrow \mathcal{O}_{X}(\Delta)$, and set $\alpha_{1}=\alpha_{2} \circ \operatorname{det} \varphi$. (Thus $\alpha_{1}=0$ if $\varphi$ is not an isomorphism.) Given $v, v^{\prime} \in E_{1}$ and $w, w^{\prime} \in E_{2}$, identify $v, v^{\prime}$ with their images in $H^{0}\left(V_{i} \otimes H_{1} \otimes L_{0}^{n}\right)$ and similarly for $w, w^{\prime}$, and let

$$
\begin{aligned}
T_{1}(\underline{V})\left(v \wedge v^{\prime}\right) & =\alpha_{1}\left(v \wedge v^{\prime}\right)=\alpha_{2} \circ \operatorname{det} \varphi\left(v \wedge v^{\prime}\right) \in H^{0}\left(\Delta \otimes H_{1}^{2} \otimes L_{0}^{2 n}\right) ; \\
T_{2}(\underline{V})\left(w \wedge w^{\prime}\right) & =\alpha_{2}\left(w \wedge w^{\prime}\right) \in H^{0}\left(\Delta \otimes H_{2}^{2} \otimes L_{0}^{2 n}\right) ; \\
T(\underline{V})(v \otimes w) & =\alpha_{2}(\varphi(v) \wedge w) \in H^{0}\left(\Delta \otimes H_{1} \otimes H_{2} \otimes L_{0}^{2 n}\right) .
\end{aligned}
$$

Changing $\alpha_{2}$ by a nonzero scalar $\lambda$ multiplies $\left(T_{1}(\underline{V}), T_{2}(\underline{V}), T(\underline{V})\right)$ by $\lambda$, so that the induced element of $\mathbb{P} U$ is well defined. Similarly, if we replace $\varphi$ by $\lambda \varphi$, then $\left(T_{1}(\underline{V}), T_{2}(\underline{V}), T(\underline{V})\right)$ is replaced by $\left(\lambda^{2} T_{1}(\underline{V}), T_{2}(\underline{V}), \lambda T(\underline{V})\right)$. It is easy to check that the map $\underline{V} \mapsto T(\underline{V})$ induces a morphism from $I$ to $\mathbb{P} U$ which is $G L\left(d_{1}\right) \times G L\left(d_{2}\right)$-equivariant. Further note that we can define $\left(T_{1}(\underline{V}), T_{2}(\underline{V}), T(\underline{V})\right)$ more generally if we are given the data $\underline{V}$ of two rank two torsion free sheaves $V_{1}$ and $V_{2}$ with $\operatorname{det} V_{i}=\Delta$, a morphism $\varphi: V_{1} \rightarrow V_{2}$, and linear maps $\psi_{i}: E_{i} \rightarrow H^{0}\left(V_{i} \otimes H_{i} \otimes L_{0}^{n}\right)$, not necessarily isomorphisms, although it is possible for $\left(T_{1}(\underline{V}), T_{2}(\underline{V}), T(\underline{V})\right)$ to be zero in this case.

We have not yet introduced the extra parameters $a_{1}$ and $a_{2}$. To do so, define $G\left(a_{1}, a_{2}\right) \subset G L\left(d_{1}\right) \times G L\left(d_{2}\right)$ as follows:

$$
G\left(a_{1}, a_{2}\right)=\left\{\left(A_{1}, A_{2}\right) \mid \operatorname{det} A_{1}^{a_{1}} \operatorname{det} A_{2}^{a_{2}}=\mathrm{Id}\right\} .
$$


Thus unlike Thaddeus we don't change the polarization or the linearization but the actual group which we use to determine stability; still our construction could probably be interpreted in his general framework. Fixing $a_{1}$ and $a_{2}$ for the rest of the discussion, we shall denote $G\left(a_{1}, a_{2}\right)$ by $G$. Since $a_{1}$ and $a_{2}$ are positive, the matrix ( $\lambda \mathrm{Id}, \lambda \mathrm{Id}$ ) lies in $G$ if and only if $\lambda$ is an $m^{\text {th }}$ root of unity, where $m=a_{1} d_{1}+a_{2} d_{2}$. Thus a quotient of $G$ by a finite group acts faithfully on $\mathbb{P U}$. Moreover, the problem of finding a good quotient of $\mathbb{P} U$ (for an appropriate open subset of $\mathbb{P} U$ ) for $G$ is the same as that of finding a good quotient of $\mathbb{P} U$ for $G L\left(d_{1}\right) \times G L\left(d_{2}\right)$, since

$$
G \cdot \mathbb{C}^{*}(\mathrm{Id}, \mathrm{Id})=G L\left(d_{1}\right) \times G L\left(d_{2}\right) .
$$

This last statement follows since $G$ clearly contains $S L\left(d_{1}\right) \times S L\left(d_{2}\right)$ and since $\mathbb{C}^{*} \times \mathbb{C}^{*}$ is generated by its diagonal subgroup and by the subgroup

$$
\left\{(\lambda, \mu): \lambda^{a_{1} d_{1}} \mu^{a_{2} d_{2}}=1\right\} .
$$

We may thus apply the general machinery of GIT to the group $G$ acting on $\mathbb{P} U$. A one parameter subgroup of $G$ is given by a basis $\left\{v_{i}\right\}$ of $E_{1}$, a basis $\left\{w_{k}\right\}$ of $E_{2}$ and weights $n_{i}, m_{k} \in \mathbb{Z}$, such that $v_{i}^{\lambda}=\lambda^{n_{i}} v_{i}, w_{k}^{\lambda}=\lambda^{m_{k}} w_{k}$, and

$$
a_{1} \sum_{i} n_{i}+a_{2} \sum_{k} m_{k}=0 .
$$

We shall always arrange our choice of basis so that $n_{1} \leq n_{2} \leq \cdots \leq n_{d_{1}}$ and $m_{1} \leq m_{2} \leq \cdots \leq m_{d_{2}}$. Given $\left(T_{1}, T_{2}, T\right) \in U$ and a one parameter subgroup of $G$ as above, we see that $\lim _{\lambda \rightarrow 0}\left(T_{1}, T_{2}, T\right)^{\lambda}=0$ if and only if $T_{1}\left(v_{i} \wedge v_{j}\right)=0$ for every pair of indices $i, j$ such that $n_{i}+n_{j} \leq 0, T_{2}\left(w_{k} \wedge w_{\ell}\right)=0$ for every pair of indices $k, \ell$ such that $m_{k}+m_{\ell} \leq 0$, and $T\left(v_{i} \otimes w_{j}\right)=0$ for every pair $i, k$ such that $n_{i}+m_{k} \leq 0$. Likewise the condition that $\lim _{\lambda \rightarrow 0}\left(T_{1}, T_{2}, T\right)^{\lambda}$ exists is similar, replacing the $\leq$ by strict inequality. Finally note that if $n_{i}+n_{j} \leq 0$, then $n_{1}+n_{j} \leq 0$, if $m_{k}+m_{\ell} \leq 0$ then $m_{1}+m_{\ell} \leq 0$, and if $n_{i}+m_{k} \leq 0$ then $n_{1}+m_{k} \leq 0$ and $n_{i}+m_{1} \leq 0$.

We then have the following:

Lemma 4.5. (i) Suppose that we are given the data $\underline{V}$ of two rank two torsion free sheaves $V_{1}$ and $V_{2}$ with $\operatorname{det} V_{i}=\Delta$, a morphism $\varphi: V_{1} \rightarrow$ $V_{2}$, and a linear map $E_{i} \rightarrow H^{0}\left(V_{i} \otimes H_{i} \otimes L_{0}^{n}\right)$, not necessarily an isomorphism. If $E_{i} \rightarrow H^{0}\left(V_{i} \otimes H_{i} \otimes L_{0}^{n}\right)$ is not injective for some $i$ or if $\varphi$ is not an isomorphism, then $\left(T_{1}(\underline{V}), T_{2}(\underline{V}), T(\underline{V})\right)$ is either zero or $G$-unstable. 
(ii) For $n$ sufficiently large depending only on $\Delta$ and $c$ and for $V$ a rank two torsion free sheaf with $\operatorname{det} V=\Delta$ and $c_{2}(V)=c, V$ is $\left(H_{1}, H_{2}, a_{1}, a_{2}\right)$ $L_{0}$-unstable if and only if $\left(T_{1}(\underline{V}), T_{2}(\underline{V}), T(\underline{V})\right)$ is $G$-unstable for all choices of data $\underline{V}$ such that $E_{i} \rightarrow H^{0}\left(V_{i} \otimes H_{i} \otimes L_{0}^{n}\right)$ is injective and $\varphi$ : $V_{1} \rightarrow V_{2} \cong V$ is an isomorphism, and $V$ is $\left(H_{1}, H_{2}, a_{1}, a_{2}\right) L_{0}$-strictly semistable if and only if $\left(T_{1}(\underline{V}), T_{2}(\underline{V}), T(\underline{V})\right)$ is $G$-strictly semistable for all such $\underline{V}$. Thus $V$ is $\left(H_{1}, H_{2}, a_{1}, a_{2}\right) L_{0}$-stable if and only if $\left(T_{1}(\underline{V}), T_{2}(\underline{V}), T(\underline{V})\right)$ is $G$-stable for all such $\underline{V}$.

Proof. First let us prove (i). We may assume that $\left(T_{1}(\underline{V}), T_{2}(\underline{V}), T(\underline{V})\right) \neq 0$. Suppose for example that $v_{1} \in E_{1} \mapsto 0 \in H^{0}\left(V_{1} \otimes H_{1} \otimes L_{0}^{n}\right)$. Complete $v_{1}$ to a basis of $E_{1}$ and choose a basis $\left\{w_{k}\right\}$ for $E_{2}$. Then $T_{1}(\underline{V})\left(v_{1} \wedge v_{i}\right)=0$ for all $i$ and $T(\underline{V})\left(v_{1} \otimes w_{k}\right)=0$ for all $k$. Define a one parameter subgroup of $G$ as follows: let $v_{1}^{\lambda}=\lambda^{-N} v_{1}, v_{i}^{\lambda}=\lambda^{a} v_{i}$ for $i>1$, and $w_{k}^{\lambda}=\lambda^{b} w_{k}$ for all $k$. Clearly $\lim _{\lambda \rightarrow 0}\left(T_{1}(\underline{V}), T_{2}(\underline{V}), T(\underline{V})\right)^{\lambda}=0$ provided that $a$ and $b$ are positive, so that $\left(T_{1}(\underline{V}), T_{2}(\underline{V}), T(\underline{V})\right)$ is $G$-unstable provided that the one parameter subgroup so constructed lies in $G$, or on other words provided that

$$
a_{1}\left(-N+a\left(d_{1}-1\right)\right)+a_{2} b d_{2}=0
$$

It thus suffices to take $a$ an arbitrary positive integer, $b=a_{1}$, and $N=$ $a\left(d_{1}-1\right)+a_{2} d_{2}$.

The argument in case $\varphi$ has a kernel is similar: in this case let $v_{1} \in \operatorname{Ker} \varphi$. Then $T_{1}(\underline{V})=0$ and $T(\underline{V})\left(v_{1} \otimes w_{k}\right)=0$ for all $k$, so that the previous argument handles this case also.

Next we show (ii). Let $p_{V \otimes H_{i}}$ be the usual normalized Hilbert polynomial of $V \otimes H_{i}$, and similarly for $p_{W \otimes H_{i}}$, where $W$ is a rank one subsheaf of $V$. Thus $p_{V \otimes H_{i}}$ and $p_{W \otimes H_{i}}$ have the same leading term. Given a polynomial $p$, let $\Delta p$ denote the difference polynomial. In our case, all of the polynomials $p$ that occur are quadratic polynomials with the same fixed degree two term. Thus if $p_{1}$ and $p_{2}$ are two such polynomials, then $p_{1}(n)>p_{2}(n)$ for all $n \gg 0$ if and only if the linear term of $p_{1}$ is greater than or equal to the linear term of $p_{2}$, and if the linear terms are equal then the constant term of $p_{1}$ is greater than the constant term of $p_{2}$. In this last case, where the linear terms are also equal, we see that $p_{1}(n)>p_{2}(n)$ for all $n \gg 0$ if and only if $p_{1}(n)>p_{2}(n)$ for some $n$. Finally the linear term of $p_{1}$ is greater than or equal to the linear term of $p_{2}$ if and only if $\Delta p_{1}(n) \geq \Delta p_{2}(n)$ for all $n$, which we shall write as $\Delta p_{1} \geq \Delta p_{2}$. Thus if $\Delta p_{1} \geq \Delta p_{2}$ and $p_{1}(n)>p_{2}(n)$ for some $n$, then $p_{1}(n)>p_{2}(n)$ for all $n \gg 0$. If $\Delta p_{1}=\Delta p_{2}$, then $p_{1}(n)>p_{2}(n)$ for some $n$ if and only if $p_{1}(n)>p_{2}(n)$ for all $n$. 
We shall show that, for sufficiently large $n$, if $V$ is $\left(H_{1}, H_{2}, a_{1}, a_{2}\right) L_{0^{-}}$ semistable and $\underline{V}$ corresponds to data where $E_{i} \rightarrow H^{0}\left(V \otimes H_{i} \otimes L_{0}^{n}\right)$ is injective and $\varphi$ is an isomorphism, then $\left(T_{1}(\underline{V}), T_{2}(\underline{V}), T(\underline{V})\right)$ is $G$-semistable. Note that $V$ is Mumford semistable. First we may choose $n$ so that $V \otimes H_{i}$ is generated by its global sections and has no higher cohomology, and so $\chi\left(V \otimes H_{i} \otimes L_{0}^{n}\right)=h^{0}\left(V \otimes H_{i} \otimes L_{0}^{n}\right)=d_{i}$. Hence, since $E_{i} \rightarrow H^{0}\left(V \otimes H_{i} \otimes L_{0}^{n}\right)$ is injective, it is an isomorphism. Let $W$ be a rank one subsheaf of $V$. Since $V \otimes H_{i}$ is Mumford semistable, $\Delta p_{W \otimes H_{i}} \leq \Delta p_{V \otimes H_{i}}$. Now the proof of (3) of Lemma 1.2 in [13] shows that there exists an $N$ so that, for all $n \geq N$, with $d_{i}$ as above, if $W$ is a rank one subsheaf of $V$ and such that $h^{0}\left(W \otimes H_{i} \otimes L_{0}^{n}\right) \geq d_{i} / 2$ for at least one $i(i=1,2)$, then in fact $\Delta p_{V \otimes H_{i}}=\Delta p_{W \otimes H_{i}}$ for all such $W$, and thus $\mu_{L_{0}}(V)=\mu_{L_{0}}(W)$. It is then easy to see that there is a twist $W \otimes H_{i} \otimes L_{0}^{-k}$, depending only on $L_{0}$ and $\Delta$, such that $h^{0}\left((V / W) \otimes H_{i} \otimes L_{0}^{-k}\right)=0$. The proof of Proposition 3.1 in [13] shows that in this case $h^{1}\left(W \otimes H_{i} \otimes L_{0}^{-k}\right)$ is bounded by $Q$, where $Q$ is some universal bound for the numbers $h^{1}\left(V \otimes H_{i} \otimes L_{0}^{-k}\right)$ as $V \otimes H_{i}$ ranges over the appropriate set of $L_{0}$-semistable sheaves. Thus by (4) of Lemma 1.2 in [13], the $W$ satisfying the condition that $h^{0}\left(W \otimes H_{i} \otimes L_{0}^{n}\right) \geq d_{i} / 2$ for at least one $i$ form a bounded family, and we may choose $n$ so large, depending only on $L_{0}, \Delta, c$, such that $h^{j}\left(W \otimes H_{i} \otimes L_{0}^{n}\right)=0$ for $j \geq 1$ and $i=1,2$.

Now suppose that $\left(T_{1}(\underline{V}), T_{2}(\underline{V}), T(\underline{V})\right)$ is $G$-unstable. Then there exists a one parameter subgroup of $G$ as above such that

$$
\lim _{\lambda \rightarrow 0}\left(T_{1}(\underline{V}), T_{2}(\underline{V}), T(\underline{V})\right)^{\lambda}=0 .
$$

Let

$$
\begin{aligned}
& s_{1}=\#\left\{j: T_{1}(\underline{V})\left(v_{1} \wedge v_{j}\right)=0\right\} \geq \max \left\{j: n_{1}+n_{j} \leq 0\right\} \\
& s_{2}=\#\left\{j: T_{2}(\underline{V})\left(w_{1} \wedge w_{\ell}\right)=0\right\} \geq \max \left\{\ell: m_{1}+m_{\ell} \leq 0\right\} .
\end{aligned}
$$

Since $a_{1} \sum_{i} n_{i}+a_{2} \sum_{k} m_{k}=0$, at least one of $n_{1}, m_{1}$ is negative. By symmetry we may assume that $n_{1}$ is negative, and that $n_{1} \leq m_{1}$. Since for $j \leq s_{1}, v_{1} \wedge v_{j}$ is zero as a section of $\operatorname{det}\left(V \otimes H_{1} \otimes L_{0}^{n}\right)$, the sections corresponding to $v_{j}, 1 \leq j \leq s_{1}$, are all sections of a rank one subsheaf $W_{1}$ of $V$. Likewise the sections $w_{\ell}, 1 \leq \ell \leq s_{2}$, if there are any such, are all sections of a rank one subsheaf $W_{2}$ of $V$. The condition that $T(\underline{V})\left(v_{1} \otimes w_{1}\right)=0$ insures that $W_{1}$ and $W_{2}$ are contained in a saturated rank one subsheaf $W$, if $s_{2} \neq 0$, otherwise we shall just take for $W$ the saturated rank one subsheaf containing $W_{1}$. Moreover $h^{0}\left(W \otimes H_{1} \otimes L_{0}^{n}\right) \geq s_{1}$ and $h^{0}\left(W \otimes H_{2} \otimes L_{0}^{n}\right) \geq s_{2}$. Suppose that we show that

$$
a_{1}\left(d_{1}-2 s_{1}\right)+a_{2}\left(d_{2}-2 s_{2}\right)<0 .
$$


Thus in particular $s_{i} \geq d_{i} / 2$ for at least one $i$. By our choice of $n$ and the previous paragraph, if $s_{i} \geq d_{i} / 2$ for at least one $i$, then $h^{0}\left(W \otimes H_{i} \otimes L_{0}^{n}\right)=$ $\chi\left(W \otimes H_{i} \otimes L_{0}^{n}\right)$ and furthermore $\mu_{L_{0}}(V)=\mu_{L_{0}}(W)$. Thus

$$
h^{0}\left(W \otimes H_{i} \otimes L_{0}^{n}\right)=\chi\left(W \otimes H_{i} \otimes L_{0}^{n}\right) \geq s_{i}
$$

for $i=1,2$ and so $p_{V ; H_{1}, H_{2}, a_{1}, a_{2}}(n)<p_{W ; H_{1}, H_{2}, a_{1}, a_{2}}(n)$. On the other hand, $p_{V ; H_{1}, H_{2}, a_{1}, a_{2}}$ and $p_{W ; H_{1}, H_{2}, a_{1}, a_{2}}$ are two quadratic polynomials with the same linear and quadratic terms (since $\mu_{L_{0}}(V)=\mu_{L_{0}}(W)$ ), and

$$
p_{V ; H_{1}, H_{2}, a_{1}, a_{2}}(n)<p_{W ; H_{1}, H_{2}, a_{1}, a_{2}}(n)
$$

for one value of $n$. Thus the constant term of $p_{W ; H_{1}, H_{2}, a_{1}, a_{2}}$ must be larger than that of $p_{V ; H_{1}, H_{2}, a_{1}, a_{2}}$. This contradicts the $\left(H_{1}, H_{2}, a_{1}, a_{2}\right) L_{0^{-}}$ semistability of $V$.

To see that $a_{1}\left(d_{1}-2 s_{1}\right)+a_{2}\left(d_{2}-2 s_{2}\right)<0$, let

$$
t_{1}=\#\left\{j: n_{j}+m_{1} \leq 0\right\} \leq s_{1} .
$$

Here $t_{1} \leq s_{1}$ since $T(\underline{V})\left(v_{j} \otimes w_{1}\right)=0$ implies that $v_{j}$ and $w_{1}$ are contained in a rank one subsheaf of $V$, necessarily $W$, and thus that $v_{1} \wedge v_{j}=0$. Let

$$
t_{2}=\#\left\{\ell: n_{1}+m_{\ell} \leq 0\right\} \leq s_{2}
$$

We have assumed that $n_{1} \leq m_{1}$. Then consider the expression

$$
a_{1} \sum_{j}\left(n_{1}+n_{j}\right)+a_{2} \sum_{\ell}\left(n_{1}+m_{\ell}\right) \text {. }
$$

On the one hand from the definition of the one parameter subgroup we have

$$
a_{1} \sum_{j}\left(n_{1}+n_{j}\right)+a_{2} \sum_{\ell}\left(n_{1}+m_{\ell}\right)=a_{1} d_{1} n_{1}+a_{2} d_{2} n_{1} .
$$

On the other hand, to estimate $\sum_{j}\left(n_{1}+n_{j}\right)$, we can ignore the positive terms where $n_{1}+n_{j} \geq 0$ and each of the $s_{1}$ negative terms are at least $n_{1}+n_{1} \geq 2 n_{1}$. Thus $\sum_{j}\left(n_{1}+n_{j}\right) \geq 2 s_{1} n_{1}$. Since $n_{1}<0$, this term is $\geq 2 s_{1} n_{1}$. Also this inequality is strict or $n_{1}+n_{i} \leq 0$ for every $i$, which would say that every section of $V \otimes H_{1} \otimes L_{0}^{n}$ is really a section of $W \otimes H_{1} \otimes L_{0}^{n}$ contradicting the fact that $V \otimes H_{1} \otimes L_{0}^{n}$ is generated by global sections. So $\sum_{j}\left(n_{1}+n_{j}\right)<2 s_{1} n_{1}$. Likewise we claim that $\sum_{\ell}\left(n_{1}+m_{\ell}\right) \geq 2 s_{2} n_{1}$. Here, to estimate $\sum_{\ell}\left(n_{1}+m_{\ell}\right)$, we may ignore the terms with $n_{1}+m_{\ell}$ positive, leaving $t_{2}$ terms $n_{1}+m_{\ell}$ which are $\leq 0$, and moreover each such term is at 
least $n_{1}+m_{1} \geq 2 n_{1}$. Thus $\sum_{\ell}\left(n_{1}+m_{\ell}\right) \geq 2 t_{2} n_{1}$, and since $t_{2} \leq s_{2}$ and $n_{1}<0$, we have $2 t_{2} n_{1} \geq 2 s_{2} n_{1}$.

Putting this together we have

$$
\begin{aligned}
a_{1} d_{1} n_{1}+a_{2} d_{2} n_{1} & =a_{1} \sum_{j}\left(n_{1}+n_{j}\right)+a_{2} \sum_{\ell}\left(n_{1}+m_{\ell}\right) \\
& >a_{1}\left(2 s_{1} n_{1}\right)+a_{2}\left(2 s_{2} n_{1}\right),
\end{aligned}
$$

so that

$$
a_{1}\left(d_{1}-2 s_{1}\right) n_{1}+a_{2}\left(d_{2}-2 s_{2}\right) n_{1}>0 .
$$

As $n_{1}<0$, we must have $a_{1}\left(d_{1}-2 s_{1}\right)+a_{2}\left(d_{2}-2 s_{2}\right)<0$, as desired.

We have thus shown that, if $\left(T_{1}(\underline{V}), T_{2}(\underline{V}), T(\underline{V})\right)$ is $G$-unstable, then $V$ is $\left(H_{1}, H_{2}, a_{1}, a_{2}\right) L_{0}$-unstable. A very similar argument handles the $G$ strictly semistable case.

Now we turn to the converse statement, that if $V$ is $\left(H_{1}, H_{2}, a_{1}, a_{2}\right)$ $L_{0}$-unstable then $\left(T_{1}(\underline{V}), T_{2}(\underline{V}), T(\underline{V})\right)$ is $G$-unstable. Suppose instead that

$$
\left(T_{1}(\underline{V}), T_{2}(\underline{V}), T(\underline{V})\right)
$$

is $G$-semistable. Let $W$ be a rank one subsheaf of $V$ such that

$$
p_{W ; H_{1}, H_{2}, a_{1}, a_{2}}(m)>p_{V ; H_{1}, H_{2}, a_{1}, a_{2}}(m)
$$

for all $m \gg 0$. We may assume that the quotient $W^{\prime}=V / W$ is torsion free. Thus $p_{W^{\prime} ; H_{1}, H_{2}, a_{1}, a_{2}}(m)<p_{V ; H_{1}, H_{2}, a_{1}, a_{2}}(m)$ for all $m \gg 0$, and so $\Delta p_{W^{\prime} \otimes H_{i}} \leq \Delta p_{V \otimes H_{i}}$. Now we have the map $E_{i} \rightarrow H^{0}\left(V \otimes H_{i} \otimes L_{0}^{n}\right)$. Consider $E_{i} \cap H^{0}\left(W \otimes H_{i} \otimes L_{0}^{n}\right) \subseteq E_{i}$. Let $\operatorname{dim} E_{i} \cap H^{0}\left(W \otimes H_{i} \otimes L_{0}^{n}\right)=s_{i}$. Suppose first that $a_{1}\left(d_{1}-2 s_{1}\right)+a_{2}\left(d_{2}-2 s_{2}\right)<0$. We claim that in this case $\left(T_{1}(\underline{V}), T_{2}(\underline{V}), T(\underline{V})\right)$ is $G$-unstable, a contradiction. To see this, choose a basis $v_{1}, \ldots, v_{d_{1}}$ for $E_{1}$ such that

$$
v_{i} \in E_{1} \cap H^{0}\left(W \otimes H_{1} \otimes L_{0}^{n}\right)
$$

for $i \leq s_{1}$, and similarly choose a basis $w_{1}, \ldots, w_{d_{2}}$ for $E_{2}$ such that $w_{k} \in$ $E_{2} \cap H^{0}\left(W \otimes H_{2} \otimes L_{0}^{n}\right)$ for $i \leq s_{2}$. Thus, if $i, j \leq s_{1}$ then $T_{1}(\underline{V})\left(v_{i} \wedge v_{j}\right)=0$; if $k, \ell \leq s_{2}$ then $T_{2}(\underline{V})\left(w_{k} \wedge w_{\ell}\right)=0$; if $i \leq s_{1}$ and $k \leq s_{2}$ then $T(\underline{V})\left(v_{i} \otimes w_{k}\right)=$ 0 .

We will try to find a one parameter subgroup of $G$ of the form

$$
v_{i}^{\lambda}= \begin{cases}\lambda^{-m} v_{i}, & \text { for } i \leq s_{1} \\ \lambda^{n} v_{i}, & \text { for } i>s_{1},\end{cases}
$$


and similarly

$$
w_{k}^{\lambda}= \begin{cases}\lambda^{-m} w_{k}, & \text { for } i \leq s_{2} \\ \lambda^{n} w_{k}, & \text { for } i>s_{2} .\end{cases}
$$

It is easy to check that $\lim _{\lambda \rightarrow 0}\left(T_{1}(\underline{V}), T_{2}(\underline{V}), T(\underline{V})\right)^{\lambda}=0$ if and only if $n>m$. What we must arrange is the condition

$$
a_{1}\left(-m s_{1}+n\left(d_{1}-s_{1}\right)\right)+a_{2}\left(-m s_{2}+n\left(d_{2}-s_{2}\right)\right)=0 .
$$

Now consider the linear function with rational coefficients

$$
f(t)=a_{1}\left(-s_{1}+t\left(d_{1}-s_{1}\right)\right)+a_{2}\left(-s_{2}+t\left(d_{2}-s_{2}\right)\right) .
$$

Since the coefficient of $t$ is strictly positive $f(t)$ is increasing, and

$$
\begin{aligned}
f(1) & =a_{1}\left(-s_{1}+\left(d_{1}-s_{1}\right)\right)+a_{2}\left(-s_{2}+\left(d_{2}-s_{2}\right)\right) \\
& =a_{1}\left(d_{1}-2 s_{1}\right)+a_{2}\left(d_{2}-2 s_{2}\right)<0 .
\end{aligned}
$$

Thus there is a rational number $t=n / m>1$ such that $f(t)=0$, and this gives the desired choice of $n$ and $m$. Thus if $a_{1}\left(d_{1}-2 s_{1}\right)+a_{2}\left(d_{2}-2 s_{2}\right)<0$, then $\left(T_{1}(\underline{V}), T_{2}(\underline{V}), T(\underline{V})\right)$ is $G$-unstable, contradicting our hypothesis.

The other possibility is that $a_{1}\left(d_{1}-2 s_{1}\right)+a_{2}\left(d_{2}-2 s_{2}\right) \geq 0$. In this case $d_{i} \geq 2 s_{i}$ for at least one $i$. Recalling that we have the quotient $W^{\prime}$ of $V$ by $W$, it then follows that for such an $i$ the image of $E_{i}$ in $H^{0}\left(W^{\prime} \otimes H_{i} \otimes L_{0}^{n}\right)$ must have dimension at least $d_{i} / 2$. Arguing as in Proposition 3.2 of [13], it then follows from Lemma 1.2 of [13] that $\Delta p_{W^{\prime} \otimes H_{i}}=\Delta p_{V \otimes H_{i}}$ and so that $V$ is Mumford $L_{0}$-semistable and $\mu_{L_{0}}(V)=\mu_{L_{0}}(W)$. Moreover, after enlarging $n$ if necessary (independently of $V$ ) we may assume that $h^{j}\left(V \otimes H_{i} \otimes L_{0}^{n}\right)=0$ for $j>0$. In particular, $d_{i}=\operatorname{dim} H^{0}\left(V \otimes H_{i} \otimes L_{0}^{n}\right)$ for $i=1,2$, and $E_{i} \rightarrow H^{0}\left(V \otimes H_{i} \otimes L_{0}^{n}\right)$ is an isomorphism; so $s_{i}=h^{0}\left(W \otimes H_{i} \otimes L_{0}^{n}\right)$. As $\mu_{L_{0}}(V)=\mu_{L_{0}}(W)$, the polynomials $p_{W ; H_{1}, H_{2}, a_{1}, a_{2}}$ and $p_{V ; H_{1}, H_{2}, a_{1}, a_{2}}$ have the same terms in degree one and two, and thus since $p_{W ; H_{1}, H_{2}, a_{1}, a_{2}}(m)>$ $p_{V ; H_{1}, H_{2}, a_{1}, a_{2}}(m)$ for some $m$ the same is true for all $m$, in particular for $m=$ $n$. Moreover, for a general choice of a smooth curve $C$ in the linear system corresponding to $L_{0}$, there is a fixed bound on the line bundle $W \otimes H_{i} \mid C$. A standard argument as in the proof of (2) of Lemma 1.2 of [13] shows that, for $n$ sufficiently large but independent of $V$, we have $H^{2}\left(W \otimes H_{i} \otimes L_{0}^{n}\right)=0$. Thus $s_{i}=h^{0}\left(W \otimes H_{i} \otimes L_{0}^{n}\right) \geq p_{W \otimes H_{i}}(n)$. It follows that

$$
\begin{aligned}
a_{1}\left(d_{1}-2 s_{1}\right)+a_{2}\left(d_{2}-2 s_{2}\right) & \leq a_{1}\left(d_{1}-2 p_{W \otimes H_{1}}(n)\right)+a_{2}\left(d_{2}-2 p_{W \otimes H_{2}}(n)\right) \\
& =2\left(p_{V ; H_{1}, H_{2}, a_{1}, a_{2}}(n)-p_{W ; H_{1}, H_{2}, a_{1}, a_{2}}(n)\right)<0
\end{aligned}
$$


This contradicts the assumption that $a_{1}\left(d_{1}-2 s_{1}\right)+a_{2}\left(d_{2}-2 s_{2}\right) \geq 0$. It then follows that $\left(T_{1}(\underline{V}), T_{2}(\underline{V}), T(\underline{V})\right)$ is $G$-unstable.

The strictly semistable case is similar.

We may now finish the proof of Theorem 4.4. Let $\mathbb{P} U_{\mathrm{ss}}$ be the set of $G$-semistable points of $\mathbb{P} U$. Let $I_{\mathrm{ss}}$ be the inverse image of $\mathbb{P} U_{\mathrm{ss}}$ under the morphism $I \rightarrow \mathbb{P} U$. Since every semistable sheaf is stable, $I_{\mathrm{ss}}$ is a $\mathbb{C}^{*}$-bundle over its image in $Q_{1} \times Q_{2}$. Moreover the representable functor corresponding to $I_{\mathrm{ss}}$ is easily seen to be formally smooth over the moduli functor. Arguments very similar to those for Lemma 4.3 and 4.5 of [13] show that the morphism $I_{\mathrm{ss}} \rightarrow \mathbb{P} U_{\mathrm{ss}}$ is one-to-one and proper, and thus in particular finite. Thus we may construct a quotient $\mathfrak{M}_{L_{0}}\left(\Delta, c ; H_{1}, H_{2}, a_{1}, a_{2}\right)$ of $I_{\text {ss }}$ by $G$. This quotient maps in a one-to-one and proper way to the GIT quotient of $\mathbb{P} U_{\mathrm{ss}}$ and is therefore projective. By the discussion at the beginning of the proof of Theorem 4.4 the points of $\mathfrak{M}_{L_{0}}\left(\Delta, c ; H_{1}, H_{2}, a_{1}, a_{2}\right)$ may be identified with isomorphism classes of $\left(H_{1}, H_{2}, a_{1}, a_{2}\right) L_{0}$-semistable rank two sheaves. Standard arguments then show that $\mathfrak{M}_{L_{0}}\left(\Delta, c ; H_{1}, H_{2}, a_{1}, a_{2}\right)$ has the usual properties of a coarse moduli space.

\section{The transition formula for Donaldson polynomial invariants.}

From now on, we will assume that the surface $X$ is rational with $-K_{X}$ effective, and will study the transition formula of Donaldson polynomial invariants:

$$
\delta_{w, p}^{X}\left(\mathcal{C}_{+}, \mathcal{C}_{-}\right)=D_{w, p}^{X}\left(\mathcal{C}_{+}\right)-D_{w, p}^{X}\left(\mathcal{C}_{-}\right)
$$

where $\mathcal{C}_{-}$and $\mathcal{C}_{+}$are two adjacent chambers separated by a single wall $W^{\zeta}$ of type $(w, p)$ or equivalently of type $(\Delta, c)$. For simplicity, we assume that the wall $W^{\zeta}$ is only represented by $\pm \zeta$ since the general case just involves additional notation. We use $\mathfrak{M}_{0}^{(k)}$ to stand for the moduli space $\mathfrak{M}_{0}^{(\zeta, k)}$. When $\ell_{\zeta}=0$, we also assume that

$$
h(\zeta)=h^{1}\left(X ; \mathcal{O}_{X}(2 F-\Delta) \neq 0\right.
$$

(see Corollary 2.7). The special case when $\ell_{\zeta}=h(\zeta)=0$ will be treated in Theorem 6.1. By Theorem 3.9 and Lemma 3.2 (ii), we have the following 
diagram:

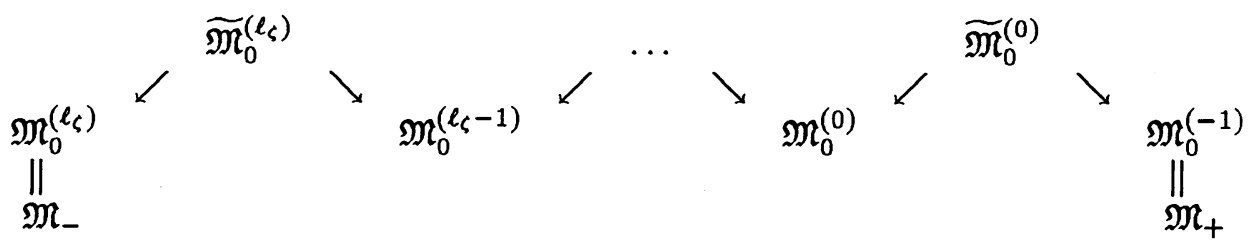

where the morphism $\widetilde{\mathfrak{M}_{0}^{(k)}} \rightarrow \mathfrak{M}_{0}^{(k)}$ is the blowup of $\mathfrak{M}_{0}^{(k)}$ at $E_{\zeta}^{\ell_{\zeta}-k, k}$, and the morphism $\widetilde{\mathfrak{M}}_{0}^{(k)} \rightarrow \mathfrak{M}_{0}^{(k-1)}$ is the blowup of $\mathfrak{M}_{0}^{(k-1)}$ at $E_{-\zeta}^{k, \ell_{\zeta}-k}$.

Next, we collect and establish some notations. Recall that in section 2 we have constructed the bundle $\mathcal{E}_{\zeta}^{\ell_{\zeta}-k, k}$ over $H_{\ell_{\zeta}-k} \times H_{k}$, where $H_{k}=\operatorname{Hilb}^{k} X$.

Notation 5.1. Let $\zeta$ define a wall of type $(w, p)$.

(i) $\lambda_{k}$ is the tautological line bundle over $E_{\zeta}^{\ell_{\zeta}-k, k}=\mathbb{P}\left(\left(\mathcal{E}_{\zeta}^{\ell_{\zeta}-k, k}\right)^{\vee}\right)$; for simplicity, we also use $\lambda_{k}$ to denote its first Chern class;

(ii) $\rho_{k}: X \times E_{\zeta}^{\ell_{\zeta}-k, k} \rightarrow X \times H_{\ell_{\zeta}-k} \times H_{k}$ is the natural projection;

(iii) $p_{k}: \widetilde{\mathfrak{M}}_{0}^{(k)} \rightarrow \mathfrak{M}_{0}^{(k)}$ is the blowup of $\mathfrak{M}_{0}^{(k)}$ at $E_{\zeta}^{\ell_{\zeta}-k, k}$;

(iv) $q_{k-1}: \widetilde{\mathfrak{M}}_{0}^{(k)} \rightarrow \mathfrak{M}_{0}^{(k-1)}$ is the contraction of $\widetilde{\mathfrak{M}}_{0}^{(k)}$ to $\mathfrak{M}_{0}^{(k-1)}$;

(v) $\mathcal{N}_{k}$ is the normal bundle of $E_{\zeta}^{\ell_{\zeta}-k, k}$ in $\mathfrak{M}_{0}^{(k)}$; by Proposition 3.7, we have

$$
\mathcal{N}_{k}=\rho_{k}^{*} \mathcal{E}_{-\zeta}^{k, \ell_{\zeta}-k} \otimes \lambda_{k}^{-1}
$$

(vi) $D_{k}=\mathbb{P}\left(\mathcal{N}_{k}^{\vee}\right)$ is the exceptional divisor in $\widetilde{\mathfrak{M}}_{0}^{(k)}$;

(vii) $\xi_{k}=\mathcal{O}_{\widetilde{\mathfrak{M}}_{0}^{(k)}}\left(-D_{k}\right) \mid D_{k}$ is the tautological line bundle on $D_{k}$; again, for simplicity, we also use $\xi_{k}$ to denote its first Chern class;

(viii) $\mu^{(k)}(\alpha)=-\frac{1}{4} p_{1}\left(\mathcal{U}^{(k)}\right) / \alpha$ where $\alpha \in H_{2}(X ; \mathbb{Z})$ and $\mathcal{U}^{(k)}$ is a universal sheaf over $X \times \mathfrak{M}_{0}^{(k)}$. Let $\mu^{\left(\ell_{\zeta}\right)}(\alpha)=\mu_{-}(\alpha)$ and that $\mu^{(-1)}(\alpha)=\mu_{+}(\alpha)$.

(ix) $\nu^{(k)}=-\frac{1}{4} p_{1}\left(\mathcal{U}^{(k)}\right) / x$ where $x \in H_{0}(X ; \mathbb{Z})$ is the natural generator. Let $\nu^{\left(\ell_{\zeta}\right)}=\nu_{-}$and that $\nu^{(-1)}=\nu_{+}$. 
Note that, in (viii) and (ix) above, the sheaf $\mathcal{U}^{(k)}$ is only defined locally in the classical topology. However, since it is defined on the level of the Quot scheme a straightforward argument shows that $p_{1}\left(\mathcal{U}^{(k)}\right)$ is a well-defined element in the rational cohomology of $X \times \mathfrak{M}_{0}^{(k)}$, at least in the complement of the universally semistable sheaves. In case there are universally semistable sheaves, then the work of $\mathrm{Li}[21]$ extends the $\mu$-map to $\mathfrak{M}_{0}^{(k)}$, at least for the two-dimensional algebraic classes. We can then extend the $\mu$-map to the 4-dimensional class via a blowup formula due to O'Grady (unpublished). Moreover, there is a universal sheaf $\mathcal{V}_{k}$ over $X \times E_{\zeta}^{\ell_{\zeta}-k, k}$. In what follows, we shall work as if there were a universal sheaf $\mathcal{U}^{(k)}$, and leave it to the reader to check that our final Chern class calculations can be verified directly even when no universal sheaf exists.

In the following lemma, we study the restrictions of $p_{k}^{*} \mu^{(k)}(\alpha)$ and $p_{k}^{*} \nu^{(k)}$ to $D_{k}$.

Lemma 5.2. Let $\alpha \in H_{2}(X ; \mathbb{Z})$ and $a=(\zeta \cdot \alpha) / 2$. Let $\tau_{1}$ and $\tau_{2}$ be the projections of $E_{\zeta}^{\ell_{\zeta}-k, k}$ to $H_{\ell_{\zeta}-k}$ and $H_{k}$ respectively. Then,

$$
\begin{aligned}
& \left(\operatorname{Id} \times p_{k}\right)^{*} c_{1}\left(\mathcal{U}^{(k)}\right) \mid\left(X \times D_{k}\right)=\pi_{1}^{*} \Delta+\left(p_{k} \mid D_{k}\right)^{*} \lambda_{k} \\
& p_{k}^{*} \mu^{(k)}(\alpha) \mid D_{k}=\left(p_{k} \mid D_{k}\right)^{*}\left[\tau_{1}^{*}\left(\left[\mathcal{Z}_{\ell_{\zeta}-k}\right] / \alpha\right)+\tau_{2}^{*}\left(\left[\mathcal{Z}_{k}\right] / \alpha\right)-a \lambda_{k}\right] \\
& p_{k}^{*} \nu^{(k)} \mid D_{k}=\frac{1}{4}\left(p_{k} \mid D_{k}\right)^{*}\left[4 \tau_{1}^{*}\left(\left[\mathcal{Z}_{\ell_{\zeta}-k}\right] / x\right)+4 \tau_{2}^{*}\left(\left[\mathcal{Z}_{k}\right] / x\right)-\lambda_{k}^{2}\right]
\end{aligned}
$$

Proof. Note that $\mathcal{U}^{(k)} \mid X \times E_{\zeta}^{\ell_{\zeta}-k, k}=\mathcal{V}_{k}$, where the sheaf $\mathcal{V}_{k}$ is constructed by Proposition 2.8 and sits in the exact sequence:

$0 \rightarrow \pi_{1}^{*} \mathcal{O}_{X}(F) \otimes \rho_{k}^{*} \pi_{1,2}^{*} I_{\mathcal{Z}_{\ell_{\zeta}-k}} \otimes \pi_{2}^{*} \lambda_{k} \rightarrow \mathcal{V}_{k} \rightarrow \pi_{1}^{*} \mathcal{O}_{X}(\Delta-F) \otimes \rho_{k}^{*} \pi_{1,3}^{*} I_{\mathcal{Z}_{k}} \rightarrow 0$

Thus, $c_{1}\left(\mathcal{V}_{k}\right)=\pi_{1}^{*} \Delta+\pi_{2}^{*} \lambda_{k}$ and $\left(\operatorname{Id} \times p_{k}\right)^{*} c_{1}\left(\mathcal{U}^{(k)}\right) \mid\left(X \times D_{k}\right)=\pi_{1}^{*} \Delta+$ $\left(p_{k} \mid D_{k}\right)^{*} \lambda_{k}$. Moreover, $c_{2}\left(\mathcal{V}_{k}\right)=\rho_{k}^{*} \pi_{1,2}^{*}\left[\mathcal{Z}_{\ell_{\zeta}-k}\right]+\rho_{k}^{*} \pi_{1,3}^{*}\left[\mathcal{Z}_{k}\right]+\left(\pi_{1}^{*} F+\pi_{2}^{*} \lambda_{k}\right)$. $\pi_{1}^{*}(\Delta-F)$. Since

$$
p_{k}^{*} \mu^{(k)}(\alpha) \mid D_{k}=\left(p_{k} \mid D_{k}\right)^{*}\left[\mu^{(k)}(\alpha) \mid E_{\zeta}^{\ell_{\zeta}-k, k}\right]=\left(p_{k} \mid D_{k}\right)^{*}\left[-\frac{1}{4} p_{1}\left(\mathcal{V}_{k}\right) / \alpha\right],
$$

we have

$$
p_{k}^{*} \mu^{(k)}(\alpha) \mid D_{k}=\left(p_{k} \mid D_{k}\right)^{*}\left[\tau_{1}^{*}\left(\left[\mathcal{Z}_{\ell_{\zeta}-k}\right] / \alpha\right)+\tau_{2}^{*}\left(\left[\mathcal{Z}_{k}\right] / \alpha\right)-a \lambda_{k}\right] .
$$


Similarly, $p_{k}^{*} \nu^{(k)} \mid D_{k}=\frac{1}{4}\left(p_{k} \mid D_{k}\right)^{*}\left[4 \tau_{1}^{*}\left(\left[\mathcal{Z}_{\ell_{\zeta}-k}\right] / x\right)+4 \tau_{2}^{*}\left(\left[\mathcal{Z}_{k}\right] / x\right)-\lambda_{k}^{2}\right]$.

It follows from the work of Morgan [26] and $\mathrm{Li}$ [21], together with unpublished work of Morgan, that $D_{w, p}^{X}\left(\mathcal{C}_{ \pm}\right)\left(\alpha^{d}\right)=\delta(\Delta) \cdot \mu_{ \pm}(\alpha)^{d}$ and

$$
D_{w, p}^{X}\left(\mathcal{C}_{ \pm}\right)\left(\alpha^{d-2}, x\right)=\delta(\Delta) \cdot \mu_{ \pm}(\alpha)^{d-2} \cdot \nu_{ \pm}
$$

where $d=-p-3, \delta(\Delta)=(-1)^{\left(\Delta^{2}+\Delta \cdot K_{X}\right) / 2}$ is the difference between the complex orientation and the standard orientation on the instanton moduli space (see [6]), and $x \in H_{0}(X ; \mathbb{Z})$ is the natural generator. Strictly speaking, their methods only handle the case of $D_{w, p}^{X}\left(\mathcal{C}_{ \pm}\right)\left(\alpha^{d}\right)$. To handle the case of $D_{w, p}^{X}\left(\mathcal{C}_{ \pm}\right)\left(\alpha^{d-2}, x\right)$, one needs a blowup formula in algebraic geometry, which has been established by O'Grady (unpublished). To compute the differences

$$
\mu_{+}(\alpha)^{d}-\mu_{-}(\alpha)^{d} \text { and } \mu_{+}(\alpha)^{d-2} \cdot \nu_{+}-\mu_{-}(\alpha)^{d-2} \cdot \nu_{-},
$$

we need to know how $\mu^{(k)}(\alpha)$ and $\mu^{(k-1)}(\alpha)$ are related, and also how $\nu^{(k)}$ and $\nu^{(k-1)}$ are related. The following lemma handles this problem.

Lemma 5.3. For $\alpha \in H_{2}(X ; \mathbb{Z})$ and the natural generator $x \in H_{0}(X ; \mathbb{Z})$, we have

$$
\begin{aligned}
& q_{k-1}^{*} \mu^{(k-1)}(\alpha)=p_{k}^{*} \mu^{(k)}(\alpha)-a D_{k} \\
& q_{k-1}^{*} \nu^{(k-1)}=p_{k}^{*} \nu^{(k)}-\frac{1}{4}\left[D_{k}^{2}+2\left(p_{k} \mid D_{k}\right)^{*} \lambda_{k}\right] .
\end{aligned}
$$

Proof. From the construction, the sheaf $\left(\operatorname{Id} \times q_{k-1}\right)^{*} \mathcal{U}^{(k-1)}$ on $X \times \widetilde{\mathfrak{M}}_{0}^{(k)}$ is the elementary modification of ( $\left.\operatorname{Id} \times p_{k}\right)^{*} \mathcal{U}^{(k)}$ along the divisor $X \times D_{k}$, using the surjection from $\left(\operatorname{Id} \times p_{k}\right)^{*} \mathcal{U}^{(k)}$ to the pullback of $\rho_{k}^{*}\left(\pi_{1}^{*} \mathcal{O}_{X}(\Delta-F) \otimes \pi_{1,3}^{*} I_{\mathcal{Z}_{k}}\right)$ :

$$
\begin{gathered}
0 \rightarrow\left(\operatorname{Id} \times q_{k-1}\right)^{*} \mathcal{U}^{(k-1)} \rightarrow\left(\operatorname{Id} \times p_{k}\right)^{*} \mathcal{U}^{(k)} \\
\rightarrow\left(\operatorname{Id} \times p_{k} \mid D_{k}\right)^{*} \rho_{k}^{*}\left(\pi_{1}^{*} \mathcal{O}_{X}(\Delta-F) \otimes \pi_{1,3}^{*} I_{\mathcal{Z}_{k}}\right) \rightarrow 0
\end{gathered}
$$

where $(2 F-\Delta)=\zeta$ and $\pi_{1}$ is the natural projection $X \times H_{\ell_{\zeta}-k} \times H_{k} \rightarrow X$. Note that $\left(\operatorname{Id} \times p_{k} \mid D_{k}\right)^{*} \rho_{k}^{*}\left(\pi_{1}^{*} \mathcal{O}_{X}(\Delta-F) \otimes \pi_{1,3}^{*} I_{\mathcal{Z}_{k}}\right)$ is a sheaf supported on $X \times D_{k}$, and that its first and second Chern classes are equal to $\left(X \times D_{k}\right)$ and $\left(X \times D_{k}^{2}\right)-\pi_{1}^{*}(\Delta-F) \cdot\left(X \times D_{k}\right)$ respectively. It follows that

$$
\begin{gathered}
\left(\operatorname{Id} \times q_{k-1}\right)^{*} c_{1}\left(\mathcal{U}^{(k-1)}\right)=\left(\operatorname{Id} \times p_{k}\right)^{*} c_{1}\left(\mathcal{U}^{(k)}\right)-\left(X \times D_{k}\right) \\
\left(\operatorname{Id} \times q_{k-1}\right)^{*} c_{2}\left(\mathcal{U}^{(k-1)}\right)=\left(\operatorname{Id} \times p_{k}\right)^{*} c_{2}\left(\mathcal{U}^{(k)}\right)-\left(\operatorname{Id} \times p_{k}\right)^{*} c_{1}\left(\mathcal{U}^{(k)}\right) \cdot\left(X \times D_{k}\right) \\
+\pi_{1}^{*}(\Delta-F) \cdot\left(X \times D_{k}\right) .
\end{gathered}
$$


By Lemma 5.2, $\left(\operatorname{Id} \times p_{k}\right)^{*} c_{1}\left(\mathcal{U}^{(k)}\right) \cdot\left(X \times D_{k}\right)=\left(\Delta \times D_{k}\right)+\left(X \times\left(p_{k} \mid D_{k}\right)^{*} \lambda_{k}\right)$. Thus,

$$
\begin{gathered}
\left(\operatorname{Id} \times q_{k-1}\right)^{*} p_{1}\left(\mathcal{U}^{(k-1)}\right)=\left(\operatorname{Id} \times p_{k}\right)^{*} p_{1}\left(\mathcal{U}^{(k)}\right)+\left(X \times D_{k}^{2}\right)-4(\Delta-F) \times D_{k} \\
+2\left(\operatorname{Id} \times p_{k}\right)^{*} c_{1}\left(\mathcal{U}^{(k)}\right) \cdot\left(X \times D_{k}\right) \\
=\left(\operatorname{Id} \times p_{k}\right)^{*} p_{1}\left(\mathcal{U}^{(k)}\right)+2(2 F-\Delta) \times D_{k} \\
+X \times\left[D_{k}^{2}+2\left(p_{k} \mid D_{k}\right)^{*} \lambda_{k}\right] .
\end{gathered}
$$

Now the conclusions follow from some straightforward calculations.

In the next two theorems, we will give formulas for the differences $\left[\mu_{+}(\alpha)\right]^{d}-\left[\mu_{-}(\alpha)\right]^{d}$ and $\left[\mu_{+}(\alpha)\right]^{d-2} \cdot \nu_{+}-\left[\mu_{-}(\alpha)\right]^{d-2} \cdot \nu_{-}$in terms of the intersections in $H_{\ell_{\zeta}-k} \times H_{k}$ and the Segre classes of the vector bundles $\mathcal{E}_{\zeta}^{\ell_{\zeta}-k, k} \oplus\left(\mathcal{E}_{-\zeta}^{k, \ell_{\zeta}-k}\right)^{\vee}$ on $H_{\ell_{\zeta}-k} \times H_{k}$, where $k=0,1, \ldots, \ell_{\zeta}$. The arguments are a little complicated, but the idea is that we are trying to get rid of the exceptional divisors $D_{k}$ as well as the Chern classes of the tautological line bundles $\xi_{k}$ and $\lambda_{k}$.

Theorem 5.4. Let $\zeta$ define a wall of type $(w, p)$, and $d=(-p-3)$. For $\alpha \in H_{2}(X ; \mathbb{Z})$, put $a=(\zeta \cdot \alpha) / 2$. Then, $\left[\mu_{+}(\alpha)\right]^{d}-\left[\mu_{-}(\alpha)\right]^{d}$ is equal to

$$
\begin{aligned}
\sum_{j=0}^{2 \ell_{\zeta}}\left(\begin{array}{l}
d \\
j
\end{array}\right) \cdot(-1)^{h(\zeta)+\ell_{\zeta}+j} \cdot a^{d-j} . \\
\quad \cdot \sum_{k=0}^{\ell_{\zeta}}\left(\left[\mathcal{Z}_{\ell_{\zeta}-k}\right] / \alpha+\left[\mathcal{Z}_{k}\right] / \alpha\right)^{j} \cdot s_{2 \ell_{\zeta}-j}\left(\mathcal{E}_{\zeta}^{\ell_{\zeta}-k, k} \oplus\left(\mathcal{E}_{-\zeta}^{k, \ell_{\zeta}-k}\right)^{\vee}\right) .
\end{aligned}
$$

Proof. By Lemma 5.3, we have $q_{k-1}^{*} \mu^{(k-1)}(\alpha)=p_{k}^{*} \mu^{(k)}(\alpha)-a D_{k}$. Since $p_{k}$ and $q_{k-1}$ are birational morphisms,

$$
\begin{aligned}
{\left[p_{k}^{*} \mu^{(k)}(\alpha)\right]^{d} } & =\left[\mu^{(k)}(\alpha)\right]^{d} \text { and } \\
{\left[q_{k-1}^{*} \mu^{(k-1)}(\alpha)\right]^{d} } & =\left[\mu^{(k-1)}(\alpha)\right]^{d} .
\end{aligned}
$$

Thus, $\left[\mu^{(k-1)}(\alpha)\right]^{d}-\left[\mu^{(k)}(\alpha)\right]^{d}$ is equal to

$$
\begin{aligned}
& \sum_{i=1}^{d}\left(\begin{array}{l}
d \\
i
\end{array}\right) \cdot\left[p_{k}^{*} \mu^{(k)}(\alpha) \mid D_{k}\right]^{d-i} \cdot\left(-D_{k} \mid D_{k}\right)^{i-1} \cdot\left(-a^{i}\right) \\
= & \sum_{i=1}^{d}\left(\begin{array}{l}
d \\
i
\end{array}\right) \cdot\left[p_{k}^{*} \mu^{(k)}(\alpha) \mid D_{k}\right]^{d-i} \cdot \xi_{k}^{i-1} \cdot\left(-a^{i}\right) .
\end{aligned}
$$


By Lemma $5.2, p_{k}^{*} \mu^{(k)}(\alpha) \mid D_{k}=\left(p_{k} \mid D_{k}\right)^{*}\left(\left[\mathcal{Z}_{\ell_{\zeta}-k}\right] / \alpha+\left[\mathcal{Z}_{k}\right] / \alpha-a \lambda_{k}\right)$. So we have

$$
\begin{aligned}
& {\left[\mu^{(k-1)}(\alpha)\right]^{d}-\left[\mu^{(k)}(\alpha)\right]^{d} } \\
= & \sum_{i=1}^{d}\left(\begin{array}{c}
d \\
i
\end{array}\right) \cdot \\
& \cdot \sum_{j=0}^{2 \ell_{\zeta}}\left(\begin{array}{c}
d-i \\
j
\end{array}\right) \cdot\left(\left[\mathcal{Z}_{\ell_{\zeta}-k}\right] / \alpha+\left[\mathcal{Z}_{k}\right] / \alpha\right)^{j} \cdot\left(-a \lambda_{k}\right)^{d-i-j} \cdot \xi_{k}^{i-1} \cdot\left(-a^{i}\right) \\
= & \sum_{j=0}^{2 \ell_{\zeta}} \sum_{i=1}^{d-j}\left(\begin{array}{c}
d \\
j
\end{array}\right) \cdot\left(\begin{array}{c}
d-j \\
i
\end{array}\right) \cdot \\
& \cdot\left(-a^{d-j}\right) \cdot\left(\left[\mathcal{Z}_{\ell_{\zeta}-k}\right] / \alpha+\left[\mathcal{Z}_{k}\right] / \alpha\right)^{j} \cdot \xi_{k}^{i-1} \cdot\left(-\lambda_{k}\right)^{d-i-j} \\
= & \sum_{j=0}^{2 \ell_{\zeta}}\left(\begin{array}{c}
d \\
j
\end{array}\right) \cdot\left(-a^{d-j}\right) \cdot\left(\left[\mathcal{Z}_{\ell_{\zeta}-k}\right] / \alpha+\left[\mathcal{Z}_{k}\right] / \alpha\right)^{j} . \\
= & \sum_{j=0}^{2 \ell_{\zeta}}\left(\begin{array}{c}
d \\
j
\end{array}\right) \cdot\left(-a^{d-j}\right) \cdot\left(\left[\mathcal{Z}_{\ell_{\zeta}-k}\right] / \alpha+\left[\mathcal{Z}_{k}\right] / \alpha\right)^{j} . \\
& \cdot \sum_{i=0}^{d-1-j}\left(\begin{array}{c}
d-j \\
i+j
\end{array}\right) \cdot \xi_{k}^{i-1} \cdot\left(-\lambda_{k}\right)^{d-i-j} \\
& \xi_{k}^{i} \cdot\left(-\lambda_{k}\right)^{d-1-j-i}
\end{aligned}
$$

Now, our formula follows from the following claim by summing $k$ from 0 to $\ell_{\zeta}$.

\section{Claim.}

$$
\begin{gathered}
\left(\left[\mathcal{Z}_{\ell_{\zeta}-k}\right] / \alpha+\left[\mathcal{Z}_{k}\right] / \alpha\right)^{j} \cdot \sum_{i=0}^{d-1-j}\left(\begin{array}{c}
d-j \\
i+1
\end{array}\right) \cdot \xi_{k}^{i} \cdot\left(-\lambda_{k}\right)^{d-1-j-i} \\
=\left(\left[\mathcal{Z}_{\ell_{\zeta}-k}\right] / \alpha+\left[\mathcal{Z}_{k}\right] / \alpha\right)^{j} \cdot(-1)^{h(\zeta)+\ell_{\zeta}+j-1} \cdot s_{2 \ell_{\zeta}-j}\left(\mathcal{E}_{\zeta}^{\ell_{\zeta}-k, k} \oplus\left(\mathcal{E}_{-\zeta}^{k, \ell_{\zeta}-k}\right)^{\vee}\right) .
\end{gathered}
$$

Proof. For simplicity, on the exceptional divisor $D_{k}$, we put

$$
\sigma_{s}=\left(\left[\mathcal{Z}_{\ell_{\zeta}-k}\right] / \alpha+\left[\mathcal{Z}_{k}\right] / \alpha\right)^{j} \cdot \sum_{i=0}^{s}\left(\begin{array}{c}
s+1 \\
i+1
\end{array}\right) \cdot \xi_{k}^{i} \cdot\left(-\lambda_{k}\right)^{s-i}
$$


So we must compute $\sigma_{d-1-j}$. Notice the relation

$$
\sigma_{s}+\lambda_{k} \cdot \sigma_{s-1}=\left(\left[\mathcal{Z}_{\ell_{\zeta}-k}\right] / \alpha+\left[\mathcal{Z}_{k}\right] / \alpha\right)^{j} \cdot\left(\xi_{k}-\lambda_{k}\right)^{s} .
$$

Thus for $0 \leq t \leq s$, we have

$$
\sigma_{s}=\left(-\lambda_{k}\right)^{t} \cdot \sigma_{s-t}+\left(\left[\mathcal{Z}_{\ell_{\zeta}-k}\right] / \alpha+\left[\mathcal{Z}_{k}\right] / \alpha\right)^{j} \cdot \sum_{i=0}^{t-1}\left(\xi_{k}-\lambda_{k}\right)^{s-i} \cdot\left(-\lambda_{k}\right)^{i} .
$$

Put $s=d-1-j$ and $t=s-N_{-\zeta}=d-1-j-N_{-\zeta}$, where $N_{-\zeta}=$ $\ell_{-\zeta}+h(-\zeta)-1=\ell_{\zeta}+h(-\zeta)-1$ as defined in Corollary 2.7. Then, $\sigma_{d-1-j}$ is equal to

$$
\begin{aligned}
\left(-\lambda_{k}\right)^{d-1-j-N_{-\zeta}} \cdot \sigma_{N_{-\zeta}}+\left(\left[\mathcal{Z}_{\ell_{\zeta}-k}\right] / \alpha\right. & \left.+\left[\mathcal{Z}_{k}\right] / \alpha\right)^{j} \\
& \cdot \sum_{i=0}^{d-2-j-N_{-\zeta}}\left(\xi_{k}-\lambda_{k}\right)^{(d-1-j)-i} \cdot\left(-\lambda_{k}\right)^{i} .
\end{aligned}
$$

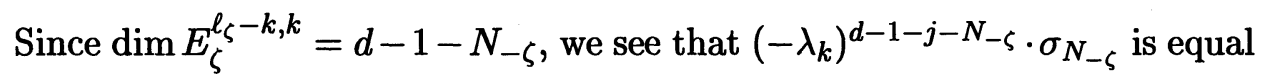
to

$$
\begin{aligned}
& \left(-\lambda_{k}\right)^{d-1-j-N_{-\zeta}} \cdot\left(\left[\mathcal{Z}_{\ell_{\zeta}-k}\right] / \alpha+\left[\mathcal{Z}_{k}\right] / \alpha\right)^{j} \cdot \sum_{i=0}^{N_{-\zeta}}\left(\begin{array}{c}
N_{-\zeta}+1 \\
i+1
\end{array}\right) \cdot \xi_{k}^{i} \cdot\left(-\lambda_{k}\right)^{N_{-\zeta}-i} \\
& =\left(-\lambda_{k}\right)^{d-1-j-N_{-\zeta}} \cdot\left(\left[\mathcal{Z}_{\ell_{\zeta}-k}\right] / \alpha+\left[\mathcal{Z}_{k}\right] / \alpha\right)^{j} \cdot \xi_{k}^{N_{-\zeta}} \\
& =\left(\left[\mathcal{Z}_{\ell_{\zeta}-k}\right] / \alpha+\left[\mathcal{Z}_{k}\right] / \alpha\right)^{j} \cdot\left(-\lambda_{k}\right)^{d-1-j-N_{-\zeta}} \cdot\left(\xi_{k}-\lambda_{k}\right)^{N_{-\zeta}}
\end{aligned}
$$

since the restriction of $\xi_{k}$ to a fiber of $D_{k} \rightarrow E_{\zeta}^{\ell_{\zeta}-k, k}$ is a hyperplane. Therefore,

$$
\sigma_{d-1-j}=\left(\left[\mathcal{Z}_{\ell_{\zeta}-k}\right] / \alpha+\left[\mathcal{Z}_{k}\right] / \alpha\right)^{j} \cdot \sum_{i=0}^{d-1-j-N_{-\zeta}}\left(\xi_{k}-\lambda_{k}\right)^{(d-1-j)-i} \cdot\left(-\lambda_{k}\right)^{i} .
$$

Now, we shall simplify $\left(\xi_{k}-\lambda_{k}\right)^{(d-1-j)-i}$. Since $\xi_{k}$ is the tautological line bundle on $D_{k}=\mathbb{P}\left(\mathcal{N}_{k}^{\vee}\right)$, the line bundle $\left(\xi_{k} \otimes \lambda_{k}^{-1}\right)$ is the tautological line bundle on

$$
\mathbb{P}\left(\mathcal{N}_{k}^{\vee} \otimes \lambda_{k}^{-1}\right)=\mathbb{P}\left[\left(\left(\rho_{k} \mid E_{\zeta}^{k, \ell_{\zeta}-k}\right)^{\vee} \mathcal{E}_{-\zeta}^{k, \ell_{\zeta}-k}\right)^{\vee}\right] .
$$

Since $N_{-\zeta}+1$ is the rank of $\mathcal{E}_{-\zeta}^{k, \ell_{\zeta}-k}$, it follows that

$$
\left(\xi_{k}-\lambda_{k}\right)^{1+N_{-\zeta}}=-\sum_{j=1}^{1+N_{-\zeta}} c_{j}\left(\mathcal{E}_{-\zeta}^{k, \ell_{\zeta}-k}\right) \cdot\left(\xi_{k}-\lambda_{k}\right)^{1+N_{-\zeta}-j}
$$


One verifies that in general, for $u^{\prime} \geq N_{-\zeta}$, one has

$$
\left(\xi_{k}-\lambda_{k}\right)^{u^{\prime}}=s_{u^{\prime}-N_{-\zeta}}\left(\mathcal{E}_{-\zeta}^{k, \ell_{\zeta}-k}\right) \cdot\left(\xi_{k}-\lambda_{k}\right)^{N_{-\zeta}}+O\left(\left(\xi_{k}-\lambda_{k}\right)^{N_{-\zeta}-1}\right)
$$

where $s_{i}\left(\mathcal{E}_{-\zeta}^{k, \ell_{\zeta}-k}\right)$ is the $i^{\text {th }}$ Segre class of $\mathcal{E}_{-\zeta}^{k, \ell_{\zeta}-k}$. Therefore, since $(d-1-$ $j)-i \geq N_{-\zeta}$, we see that $\left(\xi_{k}-\lambda_{k}\right)^{(d-1-j)-i}$ is equal to

$$
s_{d-1-j-i-N_{-\zeta}}\left(\mathcal{E}_{-\zeta}^{k, \ell_{\zeta}-k}\right) \cdot\left(\xi_{k}-\lambda_{k}\right)^{N_{-\zeta}}+O\left(\left(\xi_{k}-\lambda_{k}\right)^{N_{-\zeta}-1}\right)
$$

and that $\left(\left[\mathcal{Z}_{\ell_{\zeta}-k}\right] / \alpha+\left[\mathcal{Z}_{k}\right] / \alpha\right)^{j} \cdot\left(\xi_{k}-\lambda_{k}\right)^{(d-1-j)-i} \cdot\left(-\lambda_{k}\right)^{i}$ is equal to

$$
\begin{gathered}
\left(\left[\mathcal{Z}_{\ell_{\zeta}-k}\right] / \alpha+\left[\mathcal{Z}_{k}\right] / \alpha\right)^{j} \cdot\left[s_{d-1-j-i-N_{-\zeta}}\left(\mathcal{E}_{-\zeta}^{k, \ell_{\zeta}-k}\right) \cdot\left(\xi_{k}-\lambda_{k}\right)^{N_{-\zeta}}\right] \cdot\left(-\lambda_{k}\right)^{i} \\
=\left(\left[\mathcal{Z}_{\ell_{\zeta}-k}\right] / \alpha+\left[\mathcal{Z}_{k}\right] / \alpha\right)^{j} \cdot s_{d-1-j-i-N_{-\zeta}}\left(\mathcal{E}_{-\zeta}^{k, \ell_{\zeta}-k}\right) \cdot\left(-\lambda_{k}\right)^{i} .
\end{gathered}
$$

Next, we note that $\left(\left[\mathcal{Z}_{\ell_{\zeta}-k}\right] / \alpha+\left[\mathcal{Z}_{k}\right] / \alpha\right)^{j} \cdot s_{d-1-j-i-N_{-\zeta}}\left(\mathcal{E}_{-\zeta}^{k, \ell_{\zeta}-k}\right)$ is a cycle on $E_{\zeta}^{\ell_{\zeta}-k, k}$ pulled-back from $H_{\ell_{\zeta}-k} \times H_{k}$. So this term is zero unless $d-$ $1-i-N_{-\zeta} \leq 2 \ell_{\zeta}$, that is, $i \geq d-1-N_{-\zeta}-2 \ell_{\zeta}$. Note that by Corollary 2.7, $d-1-N_{-\zeta}-2 \ell_{\zeta}=N_{\zeta}$ and $N_{\zeta}+1=h(\zeta)+\ell_{\zeta}$ is the rank of $\mathcal{E}_{\zeta}^{\ell_{\zeta}-k, k}$. Since $\lambda_{k}$ is the tautological line bundle on $E_{\zeta}^{\ell_{\zeta}-k, k}=\mathbb{P}\left(\left(\mathcal{E}_{\zeta}^{\ell_{\zeta}-k, k}\right)^{\vee}\right)$, we see as before that

$$
\lambda_{k}^{i}=s_{i-N_{\zeta}}\left(\mathcal{E}_{\zeta}^{\ell_{\zeta}-k, k}\right) \cdot \lambda_{k}^{N_{\zeta}}+O\left(\lambda_{k}^{N_{\zeta}-1}\right) .
$$

Putting all these together, we conclude that $\sigma_{d-1-j}$ is equal to

$$
\begin{aligned}
& \left(\left[\mathcal{Z}_{\ell_{\zeta}-k}\right] / \alpha+\left[\mathcal{Z}_{k}\right] / \alpha\right)^{j} \cdot \\
& \quad \cdot \sum_{i=N_{\zeta}}^{d-1-j-N_{-\zeta}} s_{d-1-j-i-N_{-\zeta}}\left(\mathcal{E}_{-\zeta}^{k, \ell_{\zeta}-k}\right) \cdot(-1)^{i} \cdot s_{i-N_{\zeta}}\left(\mathcal{E}_{\zeta}^{\ell_{\zeta}-k, k}\right) \\
& =\left(\left[\mathcal{Z}_{\ell_{\zeta}-k}\right] / \alpha+\left[\mathcal{Z}_{k}\right] / \alpha\right)^{j} \cdot \sum_{i=0}^{2 \ell_{\zeta}-j}(-1)^{i+N_{\zeta}} \cdot s_{\left(2 \ell_{\zeta}-j\right)-i}\left(\mathcal{E}_{-\zeta}^{k, \ell_{\zeta}-k}\right) \cdot s_{i}\left(\mathcal{E}_{\zeta}^{\ell_{\zeta}-k, k}\right) \\
& =\left(\left[\mathcal{Z}_{\ell_{\zeta}-k}\right] / \alpha+\left[\mathcal{Z}_{k}\right] / \alpha\right)^{j} \cdot(-1)^{j+N_{\zeta}} . \\
& \quad \cdot \sum_{i=0}^{2 \ell_{\zeta}-j} s_{\left(2 \ell_{\zeta}-j\right)-i}\left(\left(\mathcal{E}_{-\zeta}^{k, \ell_{\zeta}-k}\right)^{\vee}\right) \cdot s_{i}\left(\mathcal{E}_{\zeta}^{\ell_{\zeta}-k, k}\right) \\
& =\left(\left[\mathcal{Z}_{\ell_{\zeta}-k}\right] / \alpha+\left[\mathcal{Z}_{k}\right] / \alpha\right)^{j} \cdot(-1)^{j+N_{\zeta}} \cdot s_{2 \ell_{\zeta}-j}\left(\mathcal{E}_{\zeta}^{\ell_{\zeta}-k, k} \oplus\left(\mathcal{E}_{-\zeta}^{k, \ell_{\zeta}-k}\right)^{\vee}\right)
\end{aligned}
$$

This completes the proof of the Theorem.

For the difference $\left[\mu_{+}(\alpha)\right]^{d-2} \cdot \nu_{+}-\left[\mu_{-}(\alpha)\right]^{d-2} \cdot \nu_{-}$, we have the following. 
Theorem 5.5. Let $\zeta$ define a wall of type $(w, p)$, and $d=-p-3$. For $\alpha \in H_{2}(X ; \mathbb{Z})$, put $a=(\zeta \cdot \alpha) / 2$. Then, $\left[\mu_{+}(\alpha)\right]^{d-2} \cdot \nu_{+}-\left[\mu_{-}(\alpha)\right]^{d-2} \cdot \nu_{-}$is equal to

$$
\begin{gathered}
\frac{1}{4} \cdot \sum_{j=0}^{2 \ell_{\zeta}}\left(\begin{array}{c}
d-2 \\
j
\end{array}\right) \cdot(-1)^{h(\zeta)+\ell_{\zeta}-1+j} \cdot a^{d-2-j} \\
\sum_{k=0}^{\ell_{\zeta}}\left(\left[\mathcal{Z}_{\ell_{\zeta}-k}\right] / \alpha+\left[\mathcal{Z}_{k}\right] / \alpha\right)^{j} \cdot\left[s_{2 \ell_{\zeta}-j}-4\left(\left[\mathcal{Z}_{\ell_{\zeta}-k}\right]+\left[\mathcal{Z}_{k}\right]\right) / x \cdot s_{2 \ell_{\zeta}-2-j}\right]
\end{gathered}
$$

where $s_{i}$ stands for the $i^{\text {th }}$ Segre class of $\mathcal{E}_{\zeta}^{\ell_{\zeta}-k, k} \oplus\left(\mathcal{E}_{-\zeta}^{k, \ell_{\zeta}-k}\right)^{\vee}$.

Proof. By Lemma 5.3, we have $q_{k-1}^{*} \mu^{(k-1)}(\alpha)=p_{k}^{*} \mu^{(k)}(\alpha)-a D_{k}$ and

$$
q_{k-1}^{*} \nu^{(k-1)}=p_{k}^{*} \nu^{(k)}-\frac{1}{4}\left[D_{k}^{2}+2\left(p_{k} \mid D_{k}\right)^{*} \lambda_{k}\right] .
$$

It follows that $\left[\mu^{(k-1)}(\alpha)\right]^{d-2} \cdot \nu^{(k-1)}-\left[\mu^{(k)}(\alpha)\right]^{d-2} \cdot \nu^{(k)}=I_{1}+I_{2}$ where

$$
\begin{aligned}
I_{1} & =\left[\mu^{(k)}(\alpha)-a D_{k}\right]^{d-2} \cdot \frac{1}{4}\left[-D_{k}^{2}-2\left(p_{k} \mid D_{k}\right)^{*} \lambda_{k}\right] \\
& =\left[\mu^{(k)}(\alpha) \mid D_{k}+a \xi_{k}\right]^{d-2} \cdot \frac{1}{4}\left(\xi_{k}-2 \lambda_{k}\right) \\
I_{2} & =\sum_{i=1}^{d-2}\left(\begin{array}{c}
d-2 \\
i
\end{array}\right) \cdot \mu^{(k)}(\alpha)^{d-2-i} \cdot\left(-a D_{k}\right)^{i} \cdot \nu^{(k)} \\
& =\sum_{i=1}^{d-2}\left(\begin{array}{c}
d-2 \\
i
\end{array}\right) \cdot\left[\mu^{(k)}(\alpha) \mid D_{k}\right]^{d-2-i} \cdot \xi_{k}^{i-1} \cdot\left(-a^{i}\right) \cdot\left(\nu^{(k)} \mid D_{k}\right) .
\end{aligned}
$$

First of all, since $\mu^{(k)}(\alpha) \mid D_{k}=\left(\left[\mathcal{Z}_{\ell_{\zeta}-k}\right] / \alpha+\left[\mathcal{Z}_{k}\right] / \alpha-a \lambda_{k}\right)$, we see that

$$
\begin{aligned}
I_{1}= & {\left[\left(\left[\mathcal{Z}_{\ell_{\zeta}-k}\right] / \alpha+\left[\mathcal{Z}_{k}\right] / \alpha\right)+a\left(\xi_{k}-\lambda_{k}\right)\right]^{d-2} \cdot \frac{1}{4}\left(\xi_{k}-2 \lambda_{k}\right) } \\
= & \frac{1}{4} \sum_{j=0}^{2 \ell_{\zeta}}\left(\begin{array}{c}
d-2 \\
j
\end{array}\right) \cdot a^{d-2-j} \cdot\left(\left[\mathcal{Z}_{\ell_{\zeta}-k}\right] / \alpha+\left[\mathcal{Z}_{k}\right] / \alpha\right)^{j} \\
& \cdot\left(\xi_{k}-\lambda_{k}\right)^{d-2-j} \cdot\left(\xi_{k}-2 \lambda_{k}\right) \\
= & \frac{1}{4} \sum_{j=0}^{2 \ell_{\zeta}}\left(\begin{array}{c}
d-2 \\
j
\end{array}\right) \cdot a^{d-2-j} \cdot\left(\left[\mathcal{Z}_{\ell_{\zeta}-k}\right] / \alpha+\left[\mathcal{Z}_{k}\right] / \alpha\right)^{j} .
\end{aligned}
$$




$$
\begin{aligned}
& {\left[\left(\xi_{k}-\lambda_{k}\right)^{d-1-j}-\lambda_{k} \cdot\left(\xi_{k}-\lambda_{k}\right)^{d-2-j}\right] } \\
&=\frac{1}{4} \sum_{j=0}^{2 \ell_{\zeta}}\left(\begin{array}{c}
d-2 \\
j
\end{array}\right) \cdot a^{d-2-j} \cdot\left(\left[\mathcal{Z}_{\ell_{\zeta}-k}\right] / \alpha+\left[\mathcal{Z}_{k}\right] / \alpha\right)^{j} . \\
& \cdot\left[s_{d-1-j-N_{-\zeta}}\left(\mathcal{E}_{-\zeta}^{k, \ell_{\zeta}-k}\right)-\lambda_{k} \cdot s_{d-2-j-N_{-\zeta}}\left(\mathcal{E}_{-\zeta}^{k, \ell_{\zeta}-k}\right)\right] .
\end{aligned}
$$

Next, by Lemma 5.2 , we have $\nu^{(k)} \mid D_{k}=\frac{1}{4}\left[4\left[\mathcal{Z}_{\ell_{\zeta}-k}\right] / x+4\left[\mathcal{Z}_{k}\right] / x-\lambda_{k}^{2}\right]$. Thus, as in the proof of Theorem 5.4, we can verify that $I_{2}$ is equal to

$$
\begin{aligned}
& \frac{1}{4}\left[4\left[\mathcal{Z}_{\ell_{\zeta}-k}\right] / x+4\left[\mathcal{Z}_{k}\right] / x-\lambda_{k}^{2}\right] \text {. } \\
& \cdot \sum_{i=1}^{d-2}\left(\begin{array}{c}
d-2 \\
i
\end{array}\right) \cdot\left[\mu^{(k)}(\alpha) \mid D_{k}\right]^{d-2-i} \cdot \xi_{k}^{i-1} \cdot\left(-a^{i}\right) \\
& =\frac{1}{4}\left[4\left[\mathcal{Z}_{\ell_{\zeta}-k}\right] / x+4\left[\mathcal{Z}_{k}\right] / x-\lambda_{k}^{2}\right] \cdot \sum_{j=0}^{2 \ell_{\zeta}}\left(\begin{array}{c}
d-2 \\
j
\end{array}\right) \cdot\left(-a^{d-2-j}\right) \cdot \\
& \cdot\left(\left[\mathcal{Z}_{\ell_{\zeta}-k}\right] / \alpha+\left[\mathcal{Z}_{k}\right] / \alpha\right)^{j} \cdot \sum_{i=0}^{2 \ell_{\zeta}+N_{\zeta}-2-j} s_{d-3-j-i-N_{-\zeta}}\left(\mathcal{E}_{-\zeta}^{k, \ell_{\zeta}-k}\right) \cdot\left(-\lambda_{k}\right)^{i} \\
& =\frac{1}{4} \sum_{j=0}^{2 \ell_{\zeta}}\left(\begin{array}{c}
d-2 \\
j
\end{array}\right) \cdot\left(-a^{d-2-j}\right) \cdot\left(\left[\mathcal{Z}_{\ell_{\zeta}-k}\right] / \alpha+\left[\mathcal{Z}_{k}\right] / \alpha\right)^{j} \\
& \cdot\left[4\left(\left[\mathcal{Z}_{\ell_{\zeta}-k}\right]+\left[\mathcal{Z}_{k}\right]\right) / x \cdot(-1)^{j+N_{\zeta}} \cdot s^{\prime}-\right. \\
& \left.-\sum_{i=0}^{2 \ell_{\zeta}+N_{\zeta}-2-j} s_{d-3-j-i-N_{-\zeta}}\left(\mathcal{E}_{-\zeta}^{k, \ell_{\zeta}-k}\right) \cdot\left(-\lambda_{k}\right)^{i+2}\right] \\
& =\frac{1}{4} \sum_{j=0}^{2 \ell_{\zeta}}\left(\begin{array}{c}
d-2 \\
j
\end{array}\right) \cdot a^{d-2-j} \cdot\left(\left[\mathcal{Z}_{\ell_{\zeta}-k}\right] / \alpha+\left[\mathcal{Z}_{k}\right] / \alpha\right)^{j} \\
& \cdot\left[\sum_{i=0}^{2 \ell_{\zeta}+N_{\zeta}-2-j} s_{d-3-j-i-N_{-\zeta}}\left(\mathcal{E}_{-\zeta}^{k, \ell_{\zeta}-k}\right) \cdot\left(-\lambda_{k}\right)^{i+2}-\right. \\
& \left.-4\left(\left[\mathcal{Z}_{\ell_{\zeta}-k}\right]+\left[\mathcal{Z}_{k}\right]\right) / x \cdot(-1)^{j+N_{\zeta}} \cdot s^{\prime}\right]
\end{aligned}
$$

where $s^{\prime}$ stands for $s_{2 \ell_{\zeta}-2-j}\left(\mathcal{E}_{\zeta}^{\ell_{\zeta}-k, k} \oplus\left(\mathcal{E}_{-\zeta}^{k, \ell_{\zeta}-k}\right)^{\vee}\right)$. Thus, $I_{1}+I_{2}$ is equal to

$$
\frac{1}{4} \sum_{j=0}^{2 \ell_{\zeta}}\left(\begin{array}{c}
d-2 \\
j
\end{array}\right) \cdot a^{d-2-j} \cdot\left(\left[\mathcal{Z}_{\ell_{\zeta}-k}\right] / \alpha+\left[\mathcal{Z}_{k}\right] / \alpha\right)^{j}
$$




$$
\begin{aligned}
& \cdot\left[\sum_{i=-2}^{2 \ell_{\zeta}+N_{\zeta}-2-j} s_{d-3-j-i-N_{-\zeta}}\left(\mathcal{E}_{-\zeta}^{k, \ell_{\zeta}-k}\right) \cdot\left(-\lambda_{k}\right)^{i+2}-\right. \\
& \left.-4\left(\left[\mathcal{Z}_{\ell_{\zeta}-k}\right]+\left[\mathcal{Z}_{k}\right]\right) / x \cdot(-1)^{j+N_{\zeta}} \cdot s^{\prime}\right] \\
& =\frac{1}{4} \sum_{j=0}^{2 \ell_{\zeta}}\left(\begin{array}{c}
d-2 \\
j
\end{array}\right) \cdot a^{d-2-j} \cdot\left(\left[\mathcal{Z}_{\ell_{\zeta}-k}\right] / \alpha+\left[\mathcal{Z}_{k}\right] / \alpha\right)^{j} \text {. } \\
& \cdot\left[(-1)^{j+N_{\zeta}}: s^{\prime \prime}-4\left(\left[\mathcal{Z}_{\ell_{\zeta}-k}\right]+\left[\mathcal{Z}_{k}\right]\right) / x \cdot(-1)^{j+N_{\zeta}} \cdot s^{\prime}\right] \\
& =\frac{1}{4} \sum_{j=0}^{2 \ell_{\zeta}}\left(\begin{array}{c}
d-2 \\
j
\end{array}\right) \cdot(-1)^{h(\zeta)+\ell_{\zeta}-1+j} \cdot a^{d-2-j} \cdot\left(\left[\mathcal{Z}_{\ell_{\zeta}-k}\right] / \alpha+\left[\mathcal{Z}_{k}\right] / \alpha\right)^{j} \text {. } \\
& \cdot\left[s^{\prime \prime}-4\left(\left[\mathcal{Z}_{\ell_{\zeta}-k}\right]+\left[\mathcal{Z}_{k}\right]\right) / x \cdot s^{\prime}\right]
\end{aligned}
$$

since $N_{\zeta}=h(\zeta)+\ell_{\zeta}-1$, where $s^{\prime \prime}$ stands for $s_{2 \ell_{\zeta}-j}\left(\mathcal{E}_{\zeta}^{\ell_{\zeta}-k, k} \oplus\left(\mathcal{E}_{-\zeta}^{k, \ell_{\zeta}-k}\right)^{\vee}\right)$. Letting $k$ run from 0 to $\ell_{\zeta}$, we obtain the desired formula.

Remark 5.6. For the sake of convenience, we record here the following relation among the Chern classes and the Segre classes of a vector bundle:

$$
s_{n}=-c_{1} \cdot s_{n-1}-c_{2} \cdot s_{n-2}-\ldots-c_{n}
$$

with the convention that $s_{0}=1$. We refer to [12] for details.

In the next section, using Theorem 5.4 and Theorem 5.5, we shall compute $\left[\mu_{+}(\alpha)\right]^{d}-\left[\mu_{-}(\alpha)\right]^{d}$ and $\left[\mu_{+}(\alpha)\right]^{d-2} \cdot \nu_{+}-\left[\mu_{-}(\alpha)\right]^{d-2} \cdot \nu_{-}$explicitly when $0 \leq \ell_{\zeta} \leq 2$. In principle, Theorem 5.4 and Theorem 5.5 give formulas for these differences in terms of certain intersections in $H_{\ell_{\zeta}-k} \times H_{k}$. However, it is difficult to evaluate these intersection numbers in general. In the following, we shall compute the term

$$
S_{j}=\sum_{k=0}^{\ell_{\zeta}}\left(\left[\mathcal{Z}_{\ell_{\zeta}-k}\right] / \alpha+\left[\mathcal{Z}_{k}\right] / \alpha\right)^{j} \cdot s_{2 \ell_{\zeta}-j}\left(\mathcal{E}_{\zeta}^{\ell_{\zeta}-k, k} \oplus\left(\mathcal{E}_{-\zeta}^{k, \ell_{\zeta}-k}\right)^{\vee}\right)
$$

in the special cases when $j=2 \ell_{\zeta}$ and $2 \ell_{\zeta}-1$. We start with a simple lemma.

Lemma 5.8. Let $\alpha, \beta \in H_{2}(X ; \mathbb{Z})$. Then

$$
\left(\left[\mathcal{Z}_{k}\right] / \alpha\right)^{2 k}=\frac{(2 k) !}{2^{k} \cdot k !} \cdot\left(\alpha^{2}\right)^{k}
$$




$$
\begin{gathered}
\left(\left[\mathcal{Z}_{k}\right] / \alpha\right)^{2 k-1} \cdot\left(\left[\mathcal{Z}_{k}\right] / \beta\right)=\frac{(2 k) !}{2^{k} \cdot k !} \cdot\left(\alpha^{2}\right)^{k-1} \cdot(\alpha \cdot \beta) \\
\left(\left[\mathcal{Z}_{k}\right] / \alpha\right)^{2 k-2} \cdot\left(\left[\mathcal{Z}_{k}\right] / \beta\right)^{2}= \\
\frac{(2 k-2) !}{2^{k-1} \cdot(k-1) !} \cdot\left(\alpha^{2}\right)^{k-1} \cdot \beta^{2}+\frac{(2 k-2) !}{2^{k-2} \cdot(k-2) !} \cdot\left(\alpha^{2}\right)^{k-2} \cdot(\alpha \cdot \beta)^{2} .
\end{gathered}
$$

Proof. The first equality is well-known (see [29] for instance). The other statements follow from the first one by considering

$$
\left(\left[\mathcal{Z}_{k}\right] / \alpha+\left[\mathcal{Z}_{k}\right] / \beta\right)^{2 k}=\frac{(2 k) !}{2^{k} \cdot k !} \cdot\left((\alpha+\beta)^{2}\right)^{k}
$$

and formally equating the terms involving $(2 k-1)$ copies of $\alpha$ and one $\beta$ or $(2 k-2)$ copies of $\alpha$ and two copies of $\beta$.

The next result computes the term (5.7) when $j=2 \ell_{\zeta}$.

Proposition 5.9. Let $\zeta$ define a wall of type $(w, p)$, and $\alpha \in H_{2}(X ; \mathbb{Z})$. Then,

$$
S_{2 \ell_{\zeta}}=\sum_{k=0}^{\ell_{\zeta}}\left(\left[\mathcal{Z}_{\ell_{\zeta}-k}\right] / \alpha+\left[\mathcal{Z}_{k}\right] / \alpha\right)^{2 \ell_{\zeta}}=\frac{\left(2 \ell_{\zeta}\right) !}{\ell_{\zeta} !} \cdot\left(\alpha^{2}\right)^{\ell_{\zeta}}
$$

Proof. This follows in a straightforward way from Lemma 5.8 (i):

$$
\begin{aligned}
& \sum_{k=0}^{\ell_{\zeta}}\left(\left[\mathcal{Z}_{\ell_{\zeta}-k}\right] / \alpha+\left[\mathcal{Z}_{k}\right] / \alpha\right)^{2 \ell_{\zeta}} \\
= & \sum_{k=0}^{\ell_{\zeta}}\left(\begin{array}{c}
2 \ell_{\zeta} \\
2 k
\end{array}\right) \cdot\left(\left[\mathcal{Z}_{\ell_{\zeta}-k}\right] / \alpha\right)^{2\left(\ell_{\zeta}-k\right)} \cdot\left(\left[\mathcal{Z}_{k}\right] / \alpha\right)^{2 k} \\
= & \sum_{k=0}^{\ell_{\zeta}}\left(\begin{array}{c}
2 \ell_{\zeta} \\
2 k
\end{array}\right) \cdot\left[\frac{\left(2 \ell_{\zeta}-2 k\right) !}{2^{\ell_{\zeta}-k} \cdot\left(\ell_{\zeta}-k\right) !} \cdot\left(\alpha^{2}\right)^{\ell_{\zeta}-k}\right] \cdot\left[\frac{(2 k) !}{2^{k} \cdot k !} \cdot\left(\alpha^{2}\right)^{k}\right] \\
= & \sum_{k=0}^{\ell_{\zeta}}\left(\begin{array}{c}
\ell_{\zeta} \\
k
\end{array}\right) \cdot \frac{\left(2 \ell_{\zeta}\right) !}{2^{\ell_{\zeta}} \cdot \ell_{\zeta} !} \cdot\left(\alpha^{2}\right)^{\ell_{\zeta}} \\
= & \frac{\left(2 \ell_{\zeta}\right) !}{\ell_{\zeta} !} \cdot\left(\alpha^{2}\right)^{\ell_{\zeta}}
\end{aligned}
$$


To compute the term (5.7) when $j=\left(2 \ell_{\zeta}-1\right)$, we study $\mathcal{E}_{-\zeta}^{k, \ell_{\zeta}-k}$ and $\mathcal{E}_{\zeta}^{\ell_{\zeta}-k, k}$, and evaluate their first Chern classes. We begin with a general lemma.

Lemma 5.10. Let $Z, W$ be codimension 2 cycles in a smooth variety $Y$.

(i) If $Z \subseteq W$, then $\operatorname{Hom}\left(I_{W}, I_{Z}\right)=\mathcal{O}_{Y}$;

(ii) If $(Z-Z \cap W)$ is open and dense in $Z$, then $\left.\operatorname{Hom}_{W}, I_{Z}\right)=I_{Z}$;

(iii) If $Z$ and $W$ are local complete intersections meeting properly, then there is an exact sequence:

$$
0 \rightarrow \operatorname{Ext}^{1}\left(I_{W}, I_{Z}\right) \rightarrow \mathcal{O}_{W} \otimes \operatorname{det} N_{W} \rightarrow \mathcal{O}_{W \cap Z} \otimes \operatorname{det} N_{W} \rightarrow 0
$$

where $N_{W}$ is the normal bundle of $W$ in $Y$;

(iv) Assume that $Z \cap W$ is nowhere dense in $W$ and that $W$ is smooth at a generic point. Then, as a sheaf on $W, \operatorname{Ext}^{1}\left(I_{W}, I_{Z}\right)$ is of rank 1 ; thus, $c_{0}\left(\operatorname{Ext}^{1}\left(I_{W}, I_{Z}\right)\right)=c_{1}\left(\operatorname{Ext}^{1}\left(I_{W}, I_{Z}\right)\right)=0, c_{2}\left(\operatorname{Ext}^{1}\left(I_{W}, I_{Z}\right)\right)=-[W]$.

Proof. (i) Applying the functor $\operatorname{Hom}\left(I_{W}, \cdot\right)$ to the exact sequence

$$
0 \rightarrow I_{Z} \rightarrow \mathcal{O}_{Y} \rightarrow \mathcal{O}_{Z} \rightarrow 0
$$

we obtain $0 \rightarrow \operatorname{Hom}\left(I_{W}, I_{Z}\right) \rightarrow \operatorname{Hom}\left(I_{W}, \mathcal{O}_{Y}\right)=\mathcal{O}_{Y}$. Thus, $\operatorname{Hom}\left(I_{W}, I_{Z}\right)$ $=I_{U}$ for some closed subscheme $U$ of $Y$. On the other hand, since $Z \subseteq W$,

$$
H^{0}\left(Y ; H o m\left(I_{W}, I_{Z}\right)\right)=\operatorname{Hom}\left(I_{W}, I_{Z}\right) \neq 0 \text {. }
$$

Thus, $U$ must be empty, and $\operatorname{Hom}\left(I_{W}, I_{Z}\right)=\mathcal{O}_{Y}$.

(ii) As in the proof of (i), $\operatorname{Hom}\left(I_{W}, I_{Z}\right)=I_{U}$ for some closed subscheme $U$ of $Y$. Applying the functor $\operatorname{Hom}\left(\cdot, I_{Z}\right)$ to the exact sequence

$$
0 \rightarrow I_{W} \rightarrow \mathcal{O}_{Y} \rightarrow \mathcal{O}_{W} \rightarrow 0
$$

we get $0 \rightarrow I_{Z} \rightarrow \operatorname{Hom}\left(I_{W}, I_{Z}\right)=I_{U} \rightarrow \operatorname{Ext} t^{1}\left(\mathcal{O}_{W}, I_{Z}\right)$. Thus, $U \subseteq Z$; moreover, since $\operatorname{Ext}^{1}\left(\mathcal{O}_{W}, I_{Z}\right)=0$ on $(X-W)$, we have $(Z-Z \cap W)=$ $(U-U \cap W)$. So

$$
(Z-Z \cap W) \subseteq U \subseteq Z
$$


Since $(Z-Z \cap W)$ is open and dense in $Z$, it follows that $U=Z$.

(iii) We begin with the local identification: let $R$ be a regular local ring, and let $Z$ and $W$ be two codimension 2 local complete intersection subschemes of $R$ meeting properly. Applying the functor $\operatorname{Hom}_{R}\left(\cdot, I_{Z}\right)$ to the Koszul resolution of $W$

$$
0 \rightarrow R \rightarrow R \oplus R \rightarrow I_{W} \rightarrow 0
$$

gives $I_{Z} \oplus I_{Z} \rightarrow I_{Z} \rightarrow \operatorname{Ext}_{R}^{1}\left(I_{W}, I_{Z}\right) \rightarrow 0$. It follows that $\operatorname{Ext}_{R}^{1}\left(I_{W}, I_{Z}\right)=$ $I_{Z} /\left(I_{Z} \cdot I_{W}\right)$. Since $Z$ and $W$ are codimension 2 local complete intersections meeting properly, we have $I_{Z} \cdot I_{W}=I_{Z} \cap I_{W}$. Thus, $\operatorname{Ext}_{R}^{1}\left(I_{W}, I_{Z}\right) \cong$ $I_{Z} /\left(I_{Z} \cap I_{W}\right)$, and we can fit it into an exact sequence

$$
0 \rightarrow \operatorname{Ext}_{R}^{1}\left(I_{W}, I_{Z}\right) \rightarrow R / I_{W} \rightarrow R /\left(I_{W}+I_{Z}\right) \rightarrow 0 .
$$

Here $\left(I_{W}+I_{Z}\right)$ corresponds to the intersection $W \cap Z$. The identification of $\operatorname{Ext}_{R}^{1}\left(I_{W}, I_{Z}\right)$ and $I_{Z} /\left(I_{Z} \cap I_{W}\right)$ is not canonical. Globally we must correct by $\operatorname{det} N_{W}$. Thus globally we have an exact sequence:

$$
0 \rightarrow \operatorname{Ext}^{1}\left(I_{W}, I_{Z}\right) \rightarrow \mathcal{O}_{W} \otimes \operatorname{det} N_{W} \rightarrow \mathcal{O}_{W \cap Z} \otimes \operatorname{det} N_{W} \rightarrow 0 .
$$

(iv) It is clear that $\operatorname{Ext}^{1}\left(I_{W}, I_{Z}\right)$ is a sheaf supported on $W$. To show that it has rank 1 as a sheaf on $W$, it suffices to verify that it has rank 1 at a generic point $w$ of $W$. Since $Z \cap W$ is nowhere dense in $W$ and $W$ is smooth at a generic point, we may assume that $w \notin Z$ and that $w$ is a smooth point of $W$. Then it follows from (iii) that $\operatorname{Ext}^{1}\left(I_{W}, I_{Z}\right)$ is of rank 1 at $w$.

Lemma 5.11. Let Hom $=\operatorname{Hom}\left(I_{\mathcal{Z}_{k}}, I_{\mathcal{Z}_{\ell^{-k}}}\right), \operatorname{Ext}^{1}=\operatorname{Ext}^{1}\left(I_{\mathcal{Z}_{k}}, I_{\mathcal{Z}_{\ell^{-k}}}\right)$, $\pi_{1}$ and $\pi_{2}$ be the projections from $X \times\left(H_{\ell_{\zeta}-k} \times H_{k}\right)$ to $X$ and $\left(H_{\ell_{\zeta}-k} \times H_{k}\right)$ respectively.

(i) There exist a row exact sequence and a column exact sequence:

$$
\begin{gathered}
0 \\
\downarrow \\
\pi_{2 *}\left(\pi_{1}^{*} \mathcal{O}_{X}(\zeta) \otimes \mathcal{O}_{\mathcal{Z}_{\ell_{\zeta}-k}}\right) \\
\downarrow \\
1 \\
0 \rightarrow \quad R^{1} \pi_{2 *}\left(\pi_{1}^{*} \mathcal{O}_{X}(\zeta) \otimes H o m\right) \rightarrow \mathcal{E}_{\zeta}^{\ell_{\zeta}-k, k} \rightarrow \pi_{2 *}\left(\pi_{1}^{*} \mathcal{O}_{X}(\zeta) \otimes E x t^{1}\right) \rightarrow 0 \\
\downarrow \\
{\left[\mathcal{O}_{H_{\ell_{\zeta}-k} \times H_{k}}\right]^{\oplus h(\zeta)}} \\
\downarrow \\
0
\end{gathered}
$$


(ii) $c_{1}\left(R^{1} \pi_{2 *}\left(\pi_{1}^{*} \mathcal{O}_{X}(\zeta) \otimes H o m\right)\right)=\left[\mathcal{Z}_{\ell_{\zeta}-k}\right] /\left(\zeta-K_{X} / 2\right)+\pi_{2 *}\left[c_{3}\left(\mathcal{O}_{\mathcal{Z}_{\ell^{-k}}}\right)\right] / 2$;

(iii) $c_{1}\left(\pi_{2 *}\left(\pi_{1}^{*} \mathcal{O}_{X}(\zeta) \otimes E x t^{1}\right)\right)=\left[\mathcal{Z}_{k}\right] /\left(\zeta-K_{X} / 2\right)+\pi_{2 *}\left[c_{3}\left(E x t^{1}\right)\right] / 2$.

Proof. (i) Note that the bundle $\mathcal{E}_{\zeta}^{\ell_{\zeta}-k, k}$ is defined as

$$
\begin{aligned}
E x t_{\pi_{2}}^{1}\left(\pi_{1}^{*} \mathcal{O}_{X}(\Delta-F) \otimes I_{\mathcal{Z}_{k}}, \pi_{1}^{*} \mathcal{O}_{X}(F)\right. & \left.\otimes I_{\mathcal{Z}_{\ell_{\zeta}-k}}\right)= \\
& =E x t_{\pi_{2}}^{1}\left(I_{\mathcal{Z}_{k}}, \pi_{1}^{*} \mathcal{O}_{X}(\zeta) \otimes I_{\mathcal{Z}_{\ell^{-k}}}\right) .
\end{aligned}
$$

Since $R^{2} \pi_{2 *}\left(\pi_{1}^{*} \mathcal{O}_{X}(\zeta) \otimes H o m\right)=0$, the row exact sequence follows from standard facts about relative Ext sheaves. To see the column exact sequence, we use Lemma 5.10 (ii) and apply the functor $\pi_{2 *}$ to the exact sequence

$$
0 \rightarrow \pi_{1}^{*} \mathcal{O}_{X}(\zeta) \otimes I_{\mathcal{Z}_{\ell_{\zeta}-k}} \rightarrow \pi_{1}^{*} \mathcal{O}_{X}(\zeta) \rightarrow \pi_{1}^{*} \mathcal{O}_{X}(\zeta) \otimes \mathcal{O}_{\mathcal{Z}_{\ell_{\zeta}-k}} \rightarrow 0 .
$$

(ii) Note that $H o m=I_{\mathcal{Z}_{\zeta^{-k}}}$ and that $R^{i} \pi_{2 *}\left(\pi_{1}^{*} \mathcal{O}_{X}(\zeta) \otimes H o m\right)=0$ for $i=0,2$. By the Grothendieck-Riemann-Roch Theorem, we have

$$
\begin{aligned}
& -\operatorname{ch}\left(R^{1} \pi_{2 *}\left(\pi_{1}^{*} \mathcal{O}_{X}(\zeta) \otimes H o m\right)\right) \\
= & \pi_{2 *}\left(\operatorname{ch}\left(\pi_{1}^{*} \mathcal{O}_{X}(\zeta) \otimes I_{\mathcal{Z}_{\zeta^{-k}}}\right) \cdot \pi_{1}^{*} \operatorname{Todd}\left(T_{X}\right)\right) \\
= & \pi_{2 *}\left(\pi_{1}^{*} \operatorname{ch}\left(\mathcal{O}_{X}(\zeta)\right) \cdot \operatorname{ch}\left(I_{\mathcal{Z}_{\ell^{-}}}\right) \cdot \pi_{1}^{*} \operatorname{Todd}\left(T_{X}\right)\right) .
\end{aligned}
$$

Now, the conclusion follows by comparing the degree 1 terms and by the fact that

$$
\begin{aligned}
\operatorname{ch}\left(I_{\mathcal{Z}_{\ell^{-k}}}\right)=1-\operatorname{ch}\left(\mathcal{O}_{\mathcal{Z}_{\ell^{-k}}}\right)=1-\left[\mathcal{Z}_{\ell_{\zeta}-k}\right] & -\frac{c_{3}\left(\mathcal{O}_{\mathcal{Z}_{\ell_{\zeta}-k}}\right)}{2}+ \\
& +(\text { terms with degree } \geq 4) .
\end{aligned}
$$

(iii) We have $R^{i} \pi_{2 *}\left(\pi_{1}^{*} \mathcal{O}_{X}(\zeta) \otimes E x t^{1}\right)=0$ for $i=1,2$. By Lemma 5.10

$$
\operatorname{ch}\left(E x t^{1}\right)=\left[\mathcal{Z}_{k}\right]+\frac{c_{3}\left(E x t^{1}\right)}{2}+(\text { terms with degree } \geq 4) .
$$

Again, using the Grothendieck-Riemann-Roch Theorem, we obtain

$$
\begin{aligned}
& \operatorname{ch}\left(\pi_{2 *}\left(\pi_{1}^{*} \mathcal{O}_{X}(\zeta) \otimes E x t^{1}\right)\right) \\
= & \pi_{2 *}\left(\operatorname{ch}\left(\pi_{1}^{*} \mathcal{O}_{X}(\zeta) \otimes E x t^{1}\right) \cdot \pi_{1}^{*} \operatorname{Todd}\left(T_{X}\right)\right) \\
= & \pi_{2 *}\left(\pi_{1}^{*} \operatorname{ch}\left(\mathcal{O}_{X}(\zeta)\right) \cdot \operatorname{ch}\left(E x t^{1}\right) \cdot \pi_{1}^{*} \operatorname{Todd}\left(T_{X}\right)\right) .
\end{aligned}
$$


Then, our conclusion follows by comparing the degree 1 terms.

Now, we can compute the term (5.7) for $j=2 \ell_{\zeta}-1$.

Proposition 5.12. Let $\alpha \in H_{2}(X ; \mathbb{Z})$ and $a=(\zeta \cdot \alpha) / 2$. Then,

$$
\begin{aligned}
S_{2 \ell_{\zeta}-1} & =\sum_{k=0}^{\ell_{\zeta}}\left(\left[\mathcal{Z}_{\ell_{\zeta}-k}\right] / \alpha+\left[\mathcal{Z}_{k}\right] / \alpha\right)^{2 \ell_{\zeta}-1} \cdot s_{1}\left(\mathcal{E}_{\zeta}^{\ell_{\zeta}-k, k} \oplus\left(\mathcal{E}_{-\zeta}^{k, \ell_{\zeta}-k}\right)^{\vee}\right) \\
& =(-4) \cdot \frac{\left(2 \ell_{\zeta}\right) !}{\ell_{\zeta} !} \cdot\left(\alpha^{2}\right)^{\ell_{\zeta}-1} \cdot a .
\end{aligned}
$$

Proof. By the symmetry between $k$ and $\left(\ell_{\zeta}-k\right)$, we see that $S_{2 \ell_{\zeta}-1}$ is equal to

$$
\begin{aligned}
\sum_{k=0}^{\ell_{\zeta}}\left(\left[\mathcal{Z}_{\ell_{\zeta}-k}\right] / \alpha+\left[\mathcal{Z}_{k}\right] / \alpha\right)^{2 \ell_{\zeta}-1} & \\
& \cdot \frac{s_{1}\left(\mathcal{E}_{\zeta}^{\ell_{\zeta}-k, k} \oplus\left(\mathcal{E}_{-\zeta}^{k, \ell_{\zeta}-k}\right)^{\vee}\right)+s_{1}\left(\mathcal{E}_{\zeta}^{k, \ell_{\zeta}-k} \oplus\left(\mathcal{E}_{-\zeta}^{\ell_{\zeta}-k, k}\right)^{\vee}\right)}{2}
\end{aligned}
$$

From Lemma 5.11, we conclude that $c_{1}\left(\mathcal{E}_{\zeta}^{\ell_{\zeta}-k, k}\right)$ is equal to

$$
\left(\left[\mathcal{Z}_{\ell_{\zeta}-k}\right]+\left[\mathcal{Z}_{k}\right]\right) /\left(\zeta-K_{X} / 2\right)+\frac{\pi_{2 *}\left[c_{3}\left(\mathcal{O}_{\mathcal{Z}_{\ell_{\zeta}-k}}\right)+c_{3}\left(\operatorname{Ext}^{1}\left(I_{\mathcal{Z}_{k}}, I_{\mathcal{Z}_{\ell_{\zeta}-k}}\right)\right)\right]}{2}
$$

Since $s_{1}\left(\mathcal{E}_{\zeta}^{\ell_{\zeta}-k, k} \oplus\left(\mathcal{E}_{-\zeta}^{k, \ell_{\zeta}-k}\right)^{\vee}\right)=c_{1}\left(\mathcal{E}_{-\zeta}^{k, \ell_{\zeta}-k}\right)-c_{1}\left(\mathcal{E}_{\zeta}^{\ell_{\zeta}-k, k}\right)$, we see that

$$
\frac{s_{1}\left(\mathcal{E}_{\zeta}^{\ell_{\zeta}-k, k} \oplus\left(\mathcal{E}_{-\zeta}^{k, \ell_{\zeta}-k}\right)^{\vee}\right)+s_{1}\left(\mathcal{E}_{\zeta}^{k, \ell_{\zeta}-k} \oplus\left(\mathcal{E}_{-\zeta}^{\ell_{\zeta}-k, k}\right)^{\vee}\right)}{2}=(-2) \cdot\left(\left[\mathcal{Z}_{\ell_{\zeta}-k}\right]+\left[\mathcal{Z}_{k}\right]\right) / \zeta
$$

where the $c_{3}$ 's are cancelled out. Therefore, by Lemma 5.8,

$$
\begin{aligned}
& S_{2 \ell_{\zeta}-1}= \sum_{k=0}^{\ell_{\zeta}}\left(\left[\mathcal{Z}_{\ell_{\zeta}-k}\right] / \alpha+\left[\mathcal{Z}_{k}\right] / \alpha\right)^{2 \ell_{\zeta}-1} \cdot(-2) \cdot\left(\left[\mathcal{Z}_{\ell_{\zeta}-k}\right] / \zeta+\left[\mathcal{Z}_{k}\right] / \zeta\right) \\
&=(-2) \cdot \sum_{k=0}^{\ell_{\zeta}}\left[\left(\begin{array}{c}
2 \ell_{\zeta}-1 \\
2 k
\end{array}\right) \cdot\left(\left[\mathcal{Z}_{\ell_{\zeta}-k}\right] / \alpha\right)^{2 \ell_{\zeta}-2 k-1} \cdot\left(\left[\mathcal{Z}_{k}\right] / \alpha\right)^{2 k} \cdot\left[\mathcal{Z}_{\ell_{\zeta}-k}\right] / \zeta\right. \\
&\left.+\left(\begin{array}{c}
2 \ell_{\zeta}-1 \\
2 k-1
\end{array}\right) \cdot\left(\left[\mathcal{Z}_{\ell_{\zeta}-k}\right] / \alpha\right)^{2 \ell_{\zeta}-2 k} \cdot\left(\left[\mathcal{Z}_{k}\right] / \alpha\right)^{2 k-1} \cdot\left[\mathcal{Z}_{k}\right] / \zeta\right]
\end{aligned}
$$




$$
\begin{aligned}
& =(-4) \cdot \sum_{k=1}^{\ell_{\zeta}}\left(\begin{array}{c}
2 \ell_{\zeta}-1 \\
2 k-1
\end{array}\right) \cdot\left(\left[\mathcal{Z}_{\ell_{\zeta}-k}\right] / \alpha\right)^{2 \ell_{\zeta}-2 k} \cdot\left(\left[\mathcal{Z}_{k}\right] / \alpha\right)^{2 k-1} \cdot\left[\mathcal{Z}_{k}\right] / \zeta \\
& =(-4) \cdot \frac{\left(2 \ell_{\zeta}\right) !}{\ell_{\zeta} !} \cdot\left(\alpha^{2}\right)^{\ell_{\zeta}-1} \cdot a
\end{aligned}
$$

It is possible, but far more complicated, to compute (5.7) for $j=2 \ell_{\zeta}-2$.

Next, we shall draw some consequences from our previous computations. Recall that $q_{X}$ denotes the intersection form of $X$, and that

$$
\delta(\Delta)=(-1)^{\frac{\Delta^{2}+\Delta \cdot K_{X}}{2}}
$$

is the difference between the complex orientation and the standard orientation on the instanton moduli space (see [6]). Adopting Kotschick's notation [17], we put

$$
\varepsilon(\zeta, \Delta)=(-1)^{\frac{(\zeta-\Delta) \cdot K_{X}}{2}}=(-1)^{\left(\frac{\zeta-\Delta}{2}\right)^{2}} .
$$

Theorem 5.13 below has already been obtained by Kotschick and Morgan [18] for any smooth 4-manifold with $b_{2}^{+}=1$.

Theorem 5.13. Let $\zeta$ define a wall of type $(w, p)$, and $d=-p-3$. Then,

$$
\begin{aligned}
{\left[\mu_{+}(\alpha)\right]^{d} } & -\left[\mu_{-}(\alpha)\right]^{d} \equiv \\
& \equiv(-1)^{h(\zeta)+\ell_{\zeta}} \cdot \frac{d !}{\ell_{\zeta} ! \cdot\left(d-2 \ell_{\zeta}\right) !} \cdot a^{d-2 \ell_{\zeta}} \cdot\left(\alpha^{2}\right)^{\ell_{\zeta}} \quad\left(\bmod a^{d-2 \ell_{\zeta}+2}\right)
\end{aligned}
$$

for $\alpha \in H_{2}(X ; \mathbb{Z})$, where $a=(\zeta \cdot \alpha) / 2$. Thus,

$$
\begin{aligned}
& \delta_{w, p}^{X}\left(\mathcal{C}_{-}, \mathcal{C}_{+}\right) \equiv \\
& \equiv(-1)^{d+\ell_{\zeta}} \cdot \varepsilon(\zeta, \Delta) \cdot \frac{d !}{\ell_{\zeta} ! \cdot\left(d-2 \ell_{\zeta}\right) !} \cdot\left(\frac{\zeta}{2}\right)^{d-2 \ell_{\zeta}} \cdot q_{X}^{\ell_{\zeta}} \quad\left(\bmod \zeta^{d-2 \ell_{\zeta}+2}\right)
\end{aligned}
$$

Proof. By Theorem 5.4 and our notation (5.7), we have

$$
\left[\mu_{+}(\alpha)\right]^{d}-\left[\mu_{-}(\alpha)\right]^{d} \equiv \sum_{j=2 \ell_{\zeta}-1}^{2 \ell_{\zeta}}\left(\begin{array}{l}
d \\
j
\end{array}\right) \cdot(-1)^{h(\zeta)+\ell_{\zeta}+j} \cdot a^{d-j} \cdot S_{j} \quad\left(\bmod a^{d-2 \ell_{\zeta}+2}\right) .
$$


By Proposition 5.12, $S_{2 \ell_{\zeta}-1}$ is divisible by $a$. Therefore, $\left[\mu_{+}(\alpha)\right]^{d}-\left[\mu_{-}(\alpha)\right]^{d} \equiv\left(\begin{array}{c}d \\ 2 \ell_{\zeta}\end{array}\right) \cdot(-1)^{h(\zeta)+\ell_{\zeta}} \cdot a^{d-2 \ell_{\zeta}} \cdot S_{2 \ell_{\zeta}} \quad\left(\bmod a^{d-2 \ell_{\zeta}+2}\right)$.

Now, the first conclusion follows from Proposition 5.9, and the second follows from the facts that $\gamma_{ \pm}\left(\alpha^{d}\right)=\delta(\Delta) \cdot \mu_{ \pm}(\alpha)^{d}$ and that $\delta(\Delta) \cdot(-1)^{h(\zeta)+\ell_{\zeta}}=(-1)^{\frac{\Delta^{2}+\Delta \cdot K_{X}}{2}} \cdot(-1)^{\frac{\left(\zeta \cdot K_{X}\right)}{2}-\frac{\zeta^{2}}{2}-1+\ell_{\zeta}}=(-1)^{d+\ell_{\zeta}} \cdot \varepsilon(\zeta, \Delta)$.

We remark that the sign in $\delta_{w, p}^{X}\left(\mathcal{C}_{-}, \mathcal{C}_{+}\right)$is indeed a homotopy invariant. (Note that the sign in Theorem 6.1.1 in [18] is not correct.)

The following is proved by using a similar method.

Theorem 5.14. Let $\zeta$ define a wall of type $(w, p)$. For $\alpha \in H_{2}(X ; \mathbb{Z})$, let $a=(\zeta \cdot \alpha) / 2$. Then, modulo $a^{d-2 \ell_{\zeta}},\left[\mu_{+}(\alpha)\right]^{d-2} \cdot \nu_{+}-\left[\mu_{-}(\alpha)\right]^{d-2} \cdot \nu_{-}$is equal to

$$
\frac{1}{4} \cdot(-1)^{h(\zeta)+\ell_{\zeta}-1} \cdot \frac{(d-2) !}{\ell_{\zeta} ! \cdot\left(d-2-2 \ell_{\zeta}\right) !} \cdot a^{d-2-2 \ell_{\zeta}} \cdot\left(\alpha^{2}\right)^{\ell_{\zeta}}
$$

Proof. By Theorem 5.5, $\left[\mu_{+}(\alpha)\right]^{d-2} \cdot \nu_{+}-\left[\mu_{-}(\alpha)\right]^{d-2} \cdot \nu_{-}$is equal to

$$
\frac{1}{4} \cdot \sum_{j=2 \ell_{\zeta}-1}^{2 \ell_{\zeta}}\left(\begin{array}{c}
d-2 \\
j
\end{array}\right) \cdot(-1)^{h(\zeta)+\ell_{\zeta}-1+j} \cdot a^{d-2-j} \cdot S_{j}
$$

modulo $a^{d-2 \ell_{\zeta}}$, where $S_{j}$ is the notation introduced in (5.7). By Proposition $5.12, S_{2 \ell_{\zeta}-1}$ is divisible by $a$; by Proposition 5.9 , we have

$$
S_{2 \ell_{\zeta}}=\frac{\left(2 \ell_{\zeta}\right) !}{\ell_{\zeta} !} \cdot\left(\alpha^{2}\right)^{\ell_{\zeta}}
$$

Therefore, modulo $a^{d-2 \ell_{\zeta}},\left[\mu_{+}(\alpha)\right]^{d-2} \cdot \nu_{+}-\left[\mu_{-}(\alpha)\right]^{d-2} \cdot \nu_{-}$is equal to

$$
\frac{1}{4} \cdot(-1)^{h(\zeta)+\ell_{\zeta}-1} \cdot \frac{(d-2) !}{\ell_{\zeta} ! \cdot\left(d-2-2 \ell_{\zeta}\right) !} \cdot a^{d-2-2 \ell_{\zeta}} \cdot\left(\alpha^{2}\right)^{\ell_{\zeta}} .
$$

6. The formulas when $\ell_{\zeta}=0,1,2$.

In this section, we shall compute $\left[\mu_{+}(\alpha)\right]^{d}-\left[\mu_{-}(\alpha)\right]^{d}$ and

$$
\left[\mu_{+}(\alpha)\right]^{d-2} \cdot \nu_{+}-\left[\mu_{-}(\alpha)\right]^{d-2} \cdot \nu_{-}
$$


by assuming that $\ell_{\zeta}=0,1,2$. Our first result, Theorem 6.1 below, was first obtained by Mong and Kotschick [17].

Theorem 6.1. Let $\zeta$ define a wall of type $(w, p)$ with $\ell_{\zeta}=0$. Then,

$$
\left[\mu_{+}(\alpha)\right]^{d}-\left[\mu_{-}(\alpha)\right]^{d}=(-1)^{h(\zeta)} \cdot\left(\frac{\zeta \cdot \alpha}{2}\right)^{d}
$$

for $\alpha \in H_{2}(X ; \mathbb{Z})$. In other words, $\delta_{w, p}^{X}\left(\mathcal{C}_{-}, \mathcal{C}_{+}\right)=(-1)^{d} \cdot \varepsilon(\zeta, \Delta) \cdot(\zeta / 2)^{d}$.

Proof. There are two cases: $h(\zeta)>0$ and $h(\zeta)=0$. In the first case when $h(\zeta)>0$, the formula follows immediately from Theorem 5.4. In the second case when $h(\zeta)=0$, we must have $\zeta^{2}=p$ and $\zeta \cdot K_{X}=\zeta^{2}+2=p+2$ by Corollary 2.7. Then $\mathfrak{M}_{+}$consists of $\mathfrak{M}_{-}$and an additional connected component $E_{-\zeta}^{0,0} \cong \mathbb{P}^{-p-3}$. We have constructed a universal sheaf $\mathcal{U}$ over $X \times E_{-\zeta}^{0,0}$ :

$$
0 \rightarrow \pi_{1}^{*} \mathcal{O}_{X}(\Delta-F) \otimes \pi_{2}^{*} \lambda \rightarrow \mathcal{U} \rightarrow \pi_{1}^{*} \mathcal{O}_{X}(F) \rightarrow 0
$$

where $F$ is the unique divisor satisfying $(2 F-\Delta)=\zeta, \lambda$ is the line bundle corresponding to a hyperplane in $E_{-\zeta}^{0,0} \cong \mathbb{P}^{-p-3}$, and $\pi_{1}$ and $\pi_{2}$ are the natural projections of $X \times E_{-\zeta}^{0,0}$. Thus for $\alpha \in H_{2}(X ; \mathbb{Z})$, we have

$$
\mu_{+}(\alpha)=\mu_{-}(\alpha)-\frac{1}{4} \cdot p_{1}(\mathcal{U}) / \alpha=\mu_{-}(\alpha)+a \lambda
$$

where $a=(\zeta \cdot \alpha) / 2$. Since $h(\zeta)=0$, we conclude that

$$
\mu_{+}(\alpha)^{d}=\mu_{-}(\alpha)^{d}+\left(\frac{\zeta \cdot \alpha}{2}\right)^{d}=\mu_{-}(\alpha)^{d}+(-1)^{h(\zeta)} \cdot\left(\frac{\zeta \cdot \alpha}{2}\right)^{d}
$$

The proof of the next result is similar to the proof of Theorem 6.1.

Theorem 6.2. Let $\zeta$ define a wall of type $(w, p)$ with $\ell_{\zeta}=0$, let $d=-p-3$. Then, for $\alpha \in H_{2}(X ; \mathbb{Z})$, we have

$$
\left[\mu_{+}(\alpha)\right]^{d-2} \cdot \nu_{+}-\left[\mu_{-}(\alpha)\right]^{d-2} \cdot \nu_{-}=\frac{1}{4} \cdot(-1)^{h(\zeta)-1} \cdot\left(\frac{\zeta \cdot \alpha}{2}\right)^{d-2}
$$


Next, we shall study the difference $\delta_{w, p}^{X}\left(\mathcal{C}_{-}, \mathcal{C}_{+}\right)$when $\ell_{\zeta}=1$. In this case, we have to know (5.7) for $j=2,1,0$. In view of Propositions 5.9 and 5.12 , it suffices to calculate (5.7) for $j=0$. The following lemma deals with this.

Lemma 6.3. Let $\zeta$ define a wall of type $(w, p)$ with $\ell_{\zeta}=1$. Then

$$
S_{0}=\sum_{k=0}^{1} s_{2}\left(\mathcal{E}_{\zeta}^{1-k, k} \oplus\left(\mathcal{E}_{-\zeta}^{k, 1-k}\right)^{\vee}\right)=\left(6 \zeta^{2}+2 K_{X}^{2}\right) .
$$

Proof. First, we compute the Chern classes of $\mathcal{E}_{\zeta}^{1,0}$. Let notations be as in Lemma 5.11, and set $\ell_{\zeta}=1$ and $k=0$ in Lemma 5.11. Then $E x t^{1}=0$. Since $\left(H_{\ell_{\zeta}-k} \times H_{k}\right)=X$, the codimension 2 cycle $\mathcal{Z}_{1}$ is exactly the diagonal in $X \times\left(H_{\ell_{\zeta}-k} \times H_{k}\right)=X \times X$. Thus, $\pi_{2 *}\left(\pi_{1}^{*} \mathcal{O}_{X}(\zeta) \otimes \mathcal{O}_{\mathcal{Z}_{\ell_{\zeta}-k}}\right)=\mathcal{O}_{X}(\zeta)$. By Lemma 5.11 (i), the bundle $\mathcal{E}_{\zeta}^{1,0}$ sits in an exact sequence:

$$
0 \rightarrow \mathcal{O}_{X}(\zeta) \rightarrow \mathcal{E}_{\zeta}^{1,0} \cong R^{1} \pi_{2 *}\left(\pi_{1}^{*} \mathcal{O}_{X}(\zeta) \otimes H o m\right) \rightarrow \mathcal{O}_{X}^{\oplus}{ }^{h(\zeta)} \rightarrow 0 .
$$

Thus, $c_{1}\left(\mathcal{E}_{\zeta}^{1,0}\right)=\zeta$ and $c_{2}\left(\mathcal{E}_{\zeta}^{1,0}\right)=0$.

Next, we compute the Chern classes of $\mathcal{E}_{\zeta}^{0,1}$. Let $\ell_{\zeta}=1$ and $k=1$ in Lemma 5.11. Then, $E x t^{1}=\operatorname{det}(N)$ where $N$ is the normal bundle of $\mathcal{Z}_{1}$ in $X \times X$. Thus,

$$
\pi_{2 *}\left(\pi_{1}^{*} \mathcal{O}_{X}(\zeta) \otimes E x t^{1}\right)=\mathcal{O}_{X}\left(\zeta-K_{X}\right) .
$$

By Lemma $5.11(\mathrm{i})$, the bundle $\mathcal{E}_{\zeta}^{0,1}$ sits in an exact sequence:

$$
0 \rightarrow \mathcal{O}_{X}^{\oplus h(\zeta)} \rightarrow \mathcal{E}_{\zeta}^{0,1} \rightarrow \mathcal{O}_{X}\left(\zeta-K_{X}\right) \rightarrow 0 .
$$

Thus, $c_{1}\left(\mathcal{E}_{\zeta}^{0,1}\right)=\zeta-K_{X}$ and $c_{2}\left(\mathcal{E}_{\zeta}^{0,1}\right)=0$. Replacing $\zeta$ by $-\zeta$ gives $c_{1}\left(\mathcal{E}_{-\zeta}^{0,1}\right)=$ $-\zeta-K_{X}$ and $c_{2}\left(\mathcal{E}_{-\zeta}^{0,1}\right)=0$. It follows that $c_{1}\left(\mathcal{E}_{\zeta}^{1,0} \oplus\left(\mathcal{E}_{-\zeta}^{0,1}\right)^{\vee}\right)=2 \zeta+K_{X}$ and that

$$
c_{2}\left(\mathcal{E}_{\zeta}^{1,0} \oplus\left(\mathcal{E}_{-\zeta}^{0,1}\right)^{\vee}\right)=\zeta \cdot\left(\zeta+K_{X}\right)=\zeta^{2}+\zeta \cdot K_{X}
$$

So we conclude that the Segre class $s_{2}\left(\mathcal{E}_{\zeta}^{1,0} \oplus\left(\mathcal{E}_{-\zeta}^{0,1}\right)^{\vee}\right)$ is equal to

$$
c_{1}\left(\mathcal{E}_{\zeta}^{1,0} \oplus\left(\mathcal{E}_{-\zeta}^{0,1}\right)^{\vee}\right)^{2}-c_{2}\left(\mathcal{E}_{\zeta}^{1,0} \oplus\left(\mathcal{E}_{-\zeta}^{0,1}\right)^{\vee}\right)=3 \zeta^{2}+3 \zeta \cdot K_{X}+K_{X}^{2} .
$$


Replacing $\zeta$ by $-\zeta$ gives $s_{2}\left(\mathcal{E}_{-\zeta}^{1,0} \oplus\left(\mathcal{E}_{\zeta}^{0,1}\right)^{\vee}\right)=3 \zeta^{2}-3 \zeta \cdot K_{X}+K_{X}^{2}$. Therefore,

$$
\begin{aligned}
S_{0} & =\sum_{k=0}^{1} s_{2}\left(\mathcal{E}_{\zeta}^{1-k, k} \oplus\left(\mathcal{E}_{-\zeta}^{k, 1-k}\right)^{\vee}\right) \\
& =s_{2}\left(\mathcal{E}_{\zeta}^{1,0} \oplus\left(\mathcal{E}_{-\zeta}^{0,1}\right)^{\vee}\right)+s_{2}\left(\mathcal{E}_{\zeta}^{0,1} \oplus\left(\mathcal{E}_{-\zeta}^{1,0}\right)^{\vee}\right) \\
& =s_{2}\left(\mathcal{E}_{\zeta}^{1,0} \oplus\left(\mathcal{E}_{-\zeta}^{0,1}\right)^{\vee}\right)+s_{2}\left(\left(\mathcal{E}_{\zeta}^{0,1}\right)^{\vee} \oplus \mathcal{E}_{-\zeta}^{1,0}\right) \\
& =\left(3 \zeta^{2}+3 \zeta \cdot K_{X}+K_{X}^{2}\right)+\left(3 \zeta^{2}-3 \zeta \cdot K_{X}+K_{X}^{2}\right) \\
& =6 \zeta^{2}+2 K_{X}^{2} .
\end{aligned}
$$

Now we can compute the difference $\delta_{w, p}^{X}\left(\mathcal{C}_{-}, \mathcal{C}_{+}\right)$when $\ell_{\zeta}=1$.

Theorem 6.4. Let $\zeta$ define a wall of type $(w, p)$ with $\ell_{\zeta}=1$. Then,

$$
\begin{aligned}
& {\left[\mu_{+}(\alpha)\right]^{d}-\left[\mu_{-}(\alpha)\right]^{d}=} \\
& \quad=(-1)^{h(\zeta)+1} \cdot\left\{d(d-1) \cdot a^{d-2} \cdot \alpha^{2}+\left(2 K_{X}^{2}+2 d+6\right) \cdot a^{d}\right\}
\end{aligned}
$$

for $\alpha \in H_{2}(X ; \mathbb{Z})$, where $a=(\zeta \cdot \alpha) / 2$. In other words, $\delta_{w, p}^{X}\left(\mathcal{C}_{-}, \mathcal{C}_{+}\right)$is equal to

$$
(-1)^{d+1} \cdot \varepsilon(\zeta, \Delta) \cdot\left\{d(d-1) \cdot\left(\frac{\zeta}{2}\right)^{d-2} \cdot q_{X}+\left(2 K_{X}^{2}+2 d+6\right) \cdot\left(\frac{\zeta}{2}\right)^{d}\right\}
$$

Proof. From 5.4, 5.9, 5.12, and 6.3, we conclude that

$$
\begin{aligned}
& {\left[\mu_{+}(\alpha)\right]^{d}-\left[\mu_{-}(\alpha)\right]^{d}} \\
& =(-1)^{h(\zeta)+1} \cdot d(d-1) \cdot a^{d-2} \cdot \alpha^{2}+(-1)^{h(\zeta)+1} \cdot 8 d \cdot a^{d} \\
& +(-1)^{h(\zeta)+1} \cdot a^{d} \cdot\left(6 \zeta^{2}+2 K_{X}^{2}\right) \\
& =(-1)^{h(\zeta)+1} \cdot\left\{d(d-1) \cdot a^{d-2} \cdot \alpha^{2}+\left(2 K_{X}^{2}+2 d+6\right) \cdot a^{d}\right\} \text {. }
\end{aligned}
$$

For $\left[\mu_{+}(\alpha)\right]^{d-2} \cdot \nu_{+}-\left[\mu_{-}(\alpha)\right]^{d-2} \cdot \nu_{-}$, we have the following. 
Theorem 6.5. Let $\zeta$ define a wall of type $(w, p)$ with $\ell_{\zeta}=1$, let $d=-p-3$. For $\alpha \in H_{2}(X ; \mathbb{Z})$, let $a=(\zeta \cdot \alpha) / 2$. Then, $\left[\mu_{+}(\alpha)\right]^{d-2} \cdot \nu_{+}-\left[\mu_{-}(\alpha)\right]^{d-2} \cdot \nu_{-}$ is equal to

$$
\frac{1}{4} \cdot(-1)^{h(\zeta)} \cdot\left[(d-2)(d-3) \cdot a^{d-4} \cdot \alpha^{2}+\left(2 K_{X}^{2}+2 d-18\right) \cdot a^{d-2}\right] .
$$

Proof. By Theorem 5.5, $\left[\mu_{+}(\alpha)\right]^{d-2} \cdot \nu_{+}-\left[\mu_{-}(\alpha)\right]^{d-2} \cdot \nu_{-}$is equal to

$$
\begin{aligned}
\frac{1}{4} \cdot \sum_{j=0}^{2}\left(\begin{array}{c}
d-2 \\
j
\end{array}\right) & \cdot(-1)^{h(\zeta)+j} \cdot a^{d-2-j} \cdot S_{j}- \\
& -(-1)^{h(\zeta)} \cdot a^{d-2} \cdot \sum_{k=0}^{1}\left(\left[\mathcal{Z}_{1-k}\right]+\left[\mathcal{Z}_{k}\right]\right) / x \\
= & \frac{1}{4} \cdot \sum_{j=0}^{2}\left(\begin{array}{c}
d-2 \\
j
\end{array}\right) \cdot(-1)^{h(\zeta)+j} \cdot a^{d-2-j} \cdot S_{j}-(-1)^{h(\zeta)} \cdot 2 a^{d-2} .
\end{aligned}
$$

By Proposition 5.9, Proposition 5.12, and Lemma 6.3, we have

$$
S_{2}=2 \alpha^{2}, S_{1}=-8 a, S_{0}=6 \zeta^{2}+2 K_{X}^{2} .
$$

Therefore, we conclude that $\left[\mu_{+}(\alpha)\right]^{d-2} \cdot \nu_{+}-\left[\mu_{-}(\alpha)\right]^{d-2} \cdot \nu_{-}$is equal to

$$
\frac{1}{4} \cdot(-1)^{h(\zeta)} \cdot\left[(d-2)(d-3) \cdot a^{d-4} \cdot \alpha^{2}+\left(2 K_{X}^{2}+2 d-18\right) \cdot a^{d-2}\right] .
$$

In the rest of this section, we assume that $\ell_{\zeta}=2$. The following standard facts about double coverings can be found in $[2,10]$.

Lemma 6.6. Let $\phi: Y_{1} \rightarrow Y_{2}$ be a double covering between two smooth projective varieties with $\phi_{*} \mathcal{O}_{Y_{1}}=\mathcal{O}_{Y_{2}} \oplus L^{-1}$ where $L$ is a line bundle on $Y_{2}$.

(i) $K_{Y_{1}}=\phi^{*}\left(K_{Y_{2}} \otimes L\right)$ and $L^{\otimes 2}=\mathcal{O}_{Y_{2}}(B)$ where $B$ is the branch locus in $Y_{2}$ and is the image of the fixed set of the involution $\iota$ on $Y_{1}$;

(ii) If $D$ is a divisor on $Y_{1}$, then $\phi_{*}\left(\mathcal{O}_{Y_{1}}(D)\right)$ is a rank 2 bundle on $Y_{2}$ with $c_{1}\left(\phi_{*}\left(\mathcal{O}_{Y_{1}}(D)\right)\right)=\phi_{*} D-L$ and

$$
c_{2}\left(\phi_{*}\left(\mathcal{O}_{Y_{1}}(D)\right)\right)=\frac{1}{2} \cdot\left[\left(\phi_{*} D\right)^{2}-\phi_{*}\left(D^{2}\right)-\phi_{*} D \cdot L\right] .
$$


Next, we recall some standard facts about the Hilbert scheme $H_{2}=$ Hilb $^{2}(X)$. Let $\Delta_{0} \subset X \times X$ be the diagonal, and let $\iota$ be the obvious involution on $\tilde{H}_{2}=\mathrm{Bl}_{\Delta_{0}}(X \times X)$, the blowup of $X \times X$ along $\Delta_{0}$. Let $E$ be the exceptional divisor of the blowup in $\tilde{H}_{2}$. Then, $H_{2}=\tilde{H}_{2} / \iota$ and the branch locus lies under $E$. Let $\tilde{\mathcal{Z}}_{2} \subset X \times \tilde{H}_{2}$ be the pullback of the codimension 2 cycle $\mathcal{Z}_{2} \subset X \times H_{2}$. Then, $\tilde{\mathcal{Z}}_{2}$ splits into a union of two cycles $\tilde{H}_{12}$ and $\tilde{H}_{13}$ in $X \times \tilde{H}_{2}$, which are the proper transforms in $X \times \tilde{H}_{2}$ of the two morphisms of $X \times X$ into $X \times(X \times X)$ : the first maps the first factor in $X \times X$ diagonally into $X \times X$ which is the product of the first and second factors in $X \times(X \times X)$, while the second maps the first factor in $X \times X$ diagonally into $X \times X$ which is the product of the first and third factors in $X \times(X \times X)$. Thus each $\tilde{H}_{1 j}$ is isomorphic to $\mathrm{Bl}_{\Delta_{0}}(X \times X)$, and the projection of each to $\tilde{H}_{2}$ is an isomorphism. If $\alpha \in H_{2}(X ; \mathbb{Z})$, then

$$
\left[\tilde{\mathcal{Z}}_{2}\right] / \alpha=\alpha \otimes 1+1 \otimes \alpha=\alpha \otimes 1+\iota^{*}(\alpha \otimes 1)
$$

where $\alpha \otimes 1$ and $1 \otimes \alpha$ are the pull-backs of $\alpha$ by the two projections of $\tilde{H}_{2}$ to $X$. Fix $x \in X$. Let $\tilde{X}_{x}$ be the pull-back of $X \times x \subset X \times X$ to $\tilde{H}_{2}$. Then, $\tilde{X}_{x}$ is isomorphic to the blow-up of $X$ at $p$ with the exceptional divisor $\left(\tilde{X}_{x} \cap E\right)$; moreover,

$$
\left[\tilde{\mathcal{Z}}_{2}\right] / x=\tilde{X}_{x}+\iota^{*} \tilde{X}_{x} .
$$

It is known (see p. 685 in [9]) that $\mathcal{Z}_{2}$ is smooth. Let $B$ be the branch locus of the natural double covering from $\mathcal{Z}_{2}$ to $H_{2}$. Then, $B \sim 2 L$ for some divisor $L$ on $H_{2}$, and the pull-back of $B \subset H_{2}$ to $\tilde{H}_{2}$ is $2 E$. Let $i: \mathcal{Z}_{2} \rightarrow X \times H_{2}$ be the embedding, and $\pi_{1}$ and $\pi_{2}$ be the natural projections of $X \times H_{2}$ to $X$ and $H_{2}$ respectively.

In the following, we compute the Chern and Segre classes of $\mathcal{E}_{\zeta}^{2-k, k}$ for $k=0,1,2$. The method is to use Lemma 5.11 together with Lemma 6.6. We start with $\mathcal{E}_{\zeta}^{2,0}$.

Lemma 6.9. $c_{3}\left(\mathcal{E}_{\zeta}^{2,0}\right)=c_{4}\left(\mathcal{E}_{\zeta}^{2,0}\right)=0, c_{1}\left(\mathcal{E}_{\zeta}^{2,0}\right)=\left[\mathcal{Z}_{2}\right] / \zeta-L$, and

$$
c_{2}\left(\mathcal{E}_{\zeta}^{2,0}\right)=\frac{1}{2}\left[\left(\left[\mathcal{Z}_{2}\right] / \zeta\right)^{2}-\zeta^{2} \cdot X_{x}-\left[\mathcal{Z}_{2}\right] / \zeta \cdot L\right]
$$

where $x$ is any point on $X$, and $X_{x}$ stands for $\left[\mathcal{Z}_{2}\right] / x$.

Proof. Let notations be as in Lemma 5.11, and let $\ell_{\zeta}=2$ and $k=0$. Then, $E x t^{1}=0$. By Lemma 5.11 (i), $\mathcal{E}_{\zeta}^{2,0}$ sits in an exact sequence

$$
0 \rightarrow\left(\pi_{2} \cdot i\right)_{*}\left(\pi_{1} \cdot i\right)^{*} \mathcal{O}_{X}(\zeta) \rightarrow \mathcal{E}_{\zeta}^{2,0} \rightarrow\left[\mathcal{O}_{H_{2}}\right]^{\oplus h(\zeta)} \rightarrow 0 .
$$


Since $\left(\pi_{2} \cdot i\right)_{*}\left(\pi_{1} \cdot i\right)^{*} \mathcal{O}_{X}(\zeta)$ has rank $2, c_{3}\left(\mathcal{E}_{\zeta}^{2,0}\right)=c_{4}\left(\mathcal{E}_{\zeta}^{2,0}\right)=0$. By Lemma 6.6 (ii),

$$
c_{1}\left(\mathcal{E}_{\zeta}^{2,0}\right)=\left(\pi_{2} \cdot i\right)_{*}\left(\pi_{1} \cdot i\right)^{*} \zeta-L=\left[\mathcal{Z}_{2}\right] / \zeta-L
$$

since $\left(\pi_{2} \cdot i\right)_{*}\left(\pi_{1} \cdot i\right)^{*} \zeta=\left[\mathcal{Z}_{2}\right] / \zeta ;$ moreover, we have

$$
\begin{aligned}
c_{2}\left(\mathcal{E}_{\zeta}^{2,0}\right)= & \frac{1}{2}\left[\left(\left(\pi_{2} \cdot i\right)_{*}\left(\pi_{1} \cdot i\right)^{*} \zeta\right)^{2}-\left(\pi_{2} \cdot i\right)_{*}\left(\left(\pi_{1} \cdot i\right)^{*} \zeta\right)^{2}-\right. \\
& \left.\quad-\left(\pi_{2} \cdot i\right)_{*}\left(\pi_{1} \cdot i\right)^{*} \zeta \cdot L\right] \\
= & \frac{1}{2}\left[\left(\left[\mathcal{Z}_{2}\right] / \zeta\right)^{2}-\zeta^{2} \cdot X_{x}-\left[\mathcal{Z}_{2}\right] / \zeta \cdot L\right]
\end{aligned}
$$

since $\left(\pi_{2} \cdot i\right)_{*}\left(\left(\pi_{1} \cdot i\right)^{*} \zeta\right)^{2}=\zeta^{2} \cdot\left(\pi_{2} \cdot i\right)_{*}\left(\pi_{1} \cdot i\right)^{*} x=\zeta^{2} \cdot\left[\mathcal{Z}_{2}\right] / x=\zeta^{2} \cdot X_{x}$.

The following follows from Lemma 6.9 and Remark 5.6.

Corollary 6.10. The Segre classes of the bundle $\mathcal{E}_{\zeta}^{2,0}$ are given by

$$
\begin{aligned}
& s_{1}\left(\mathcal{E}_{\zeta}^{2,0}\right)=L-\left[\mathcal{Z}_{2}\right] / \zeta \\
& s_{2}\left(\mathcal{E}_{\zeta}^{2,0}\right)=\frac{1}{2}\left[\left[\left[\mathcal{Z}_{2}\right] / \zeta\right]^{2}-3\left[\mathcal{Z}_{2}\right] / \zeta \cdot L+2 L^{2}+\zeta^{2} \cdot X_{x}\right] \\
& s_{3}\left(\mathcal{E}_{\zeta}^{2,0}\right)=\left[\left[\mathcal{Z}_{2}\right] / \zeta\right]^{2} \cdot L-2\left[\mathcal{Z}_{2}\right] / \zeta \cdot L^{2}+L^{3}-\zeta^{2} \cdot X_{x} \cdot\left[\mathcal{Z}_{2}\right] / \zeta+\zeta^{2} \cdot X_{x} \cdot L \\
& s_{4}\left(\mathcal{E}_{\zeta}^{2,0}\right)=\frac{\left(\zeta^{2}\right)^{2}}{2}-5 \zeta^{2}-\frac{5}{2} \zeta \cdot K_{X}+\left(6 \chi\left(\mathcal{O}_{X}\right)-K_{X}^{2}\right) .
\end{aligned}
$$

Here we have identified degree 4 classes with the corresponding integers.

Proof. Since the computation is straightforward, we only calculate $s_{4}\left(\mathcal{E}_{\zeta}^{2,0}\right)$. For simplicity, let $c_{i}$ denote the $i^{\text {th }}$ Chern class of $\mathcal{E}_{\zeta}^{2,0}$. Note that $c_{3}=c_{4}=0$ by Lemma 6.9 . Thus, $s_{4}\left(\mathcal{E}_{\zeta}^{2,0}\right)=c_{1}^{4}-3 c_{1}^{2} c_{2}+c_{2}^{2}$ by Remark 5.6. Therefore,

$$
\begin{gathered}
s_{4}\left(\mathcal{E}_{\zeta}^{2,0}\right)=\left(\left[\mathcal{Z}_{2}\right] / \zeta-L\right)^{4}-3\left(\left[\mathcal{Z}_{2}\right] / \zeta-L\right)^{2} \cdot \frac{1}{2}\left[\left(\left[\mathcal{Z}_{2}\right] / \zeta\right)^{2}-\zeta^{2} \cdot X_{x}\right. \\
\left.-\left[\mathcal{Z}_{2}\right] / \zeta \cdot L\right]+\frac{1}{4}\left[\left(\left[\mathcal{Z}_{2}\right] / \zeta\right)^{2}-\zeta^{2} \cdot X_{x}-\left[\mathcal{Z}_{2}\right] / \zeta \cdot L\right]^{2} \\
=L^{4}-\frac{5}{2} \cdot\left[\mathcal{Z}_{2}\right] / \zeta \cdot L^{3}+\frac{7}{4} \cdot\left(\left[\mathcal{Z}_{2}\right] / \zeta\right)^{2} \cdot L^{2}+\frac{3}{2} \zeta^{2} \cdot X_{x} \cdot L^{2} \\
-\frac{1}{4}\left(\left[\mathcal{Z}_{2}\right] / \zeta\right)^{4}+\frac{1}{4}\left(\zeta^{2}\right)^{2} \cdot X_{x}^{2}+\zeta^{2} \cdot\left(\left[\mathcal{Z}_{2}\right] / \zeta\right)^{2} \cdot X_{x}
\end{gathered}
$$

since $\left(\left[\mathcal{Z}_{2}\right] / \zeta\right)^{3} \cdot L=0=\left[\mathcal{Z}_{2}\right] / \zeta \cdot L \cdot X_{x}$. Now, we need a claim. 
Claim. Let $\alpha, \beta \in H_{2}(X ; \mathbb{Z})$. Then, we have the following:

(i) $\left[\mathcal{Z}_{2}\right] / \alpha \cdot\left[\mathcal{Z}_{2}\right] / \beta \cdot X_{x}=\alpha \cdot \beta$;

(ii) $X_{x}^{2}=1$;

(iii) $X_{x} \cdot L^{2}=-1$;

(iv) $L^{4}=6 \chi\left(\mathcal{O}_{X}\right)-K_{X}^{2}$;

(v) $\left[\mathcal{Z}_{2}\right] / \alpha \cdot L^{3}=\alpha \cdot K_{X}$;

(vi) $\left[\mathcal{Z}_{2}\right] / \alpha \cdot\left[\mathcal{Z}_{2}\right] / \beta \cdot L^{2}=-2(\alpha \cdot \beta)$.

Proof. Let $\pi: \tilde{H}_{2} \rightarrow H_{2}=\tilde{H}_{2} / \iota$ be the quotient map. By $(6.8)$, we have

$$
\pi^{*} X_{x}=\pi^{*}\left(\left[\mathcal{Z}_{2}\right] / x\right)=\left[\tilde{\mathcal{Z}}_{2}\right] / x=\left(\tilde{X}_{x}+\iota^{*} \tilde{X}_{x}\right)
$$

(i) Recall from (6.7) that $\pi^{*}\left(\left[\mathcal{Z}_{2}\right] / \alpha\right)=\left[\tilde{\mathcal{Z}}_{2}\right] / \alpha=\alpha \otimes 1+1 \otimes \alpha$. Thus,

$$
\begin{aligned}
{\left[\mathcal{Z}_{2}\right] / \alpha \cdot\left[\mathcal{Z}_{2}\right] / \beta \cdot X_{x} } & =\frac{1}{2} \cdot \pi^{*}\left(\left[\mathcal{Z}_{2}\right] / \alpha\right) \cdot \pi^{*}\left(\left[\mathcal{Z}_{2}\right] / \beta\right) \cdot \pi^{*} X_{x} \\
& =\frac{1}{2} \cdot(\alpha \otimes 1+1 \otimes \alpha) \cdot(\beta \otimes 1+1 \otimes \beta) \cdot\left(\tilde{X}_{x}+\iota^{*} \tilde{X}_{x}\right) \\
& =\alpha \cdot \beta .
\end{aligned}
$$

(ii) Let $x_{1} \in X$ be a point different from $x$. Then,

$$
\begin{aligned}
X_{x}^{2} & =X_{x} \cdot X_{x_{1}}=\frac{1}{2} \cdot \pi^{*}\left(X_{x}\right) \cdot \pi^{*}\left(X_{x_{1}}\right) \\
& =\frac{1}{2} \cdot\left(\tilde{X}_{x}+\iota^{*} \tilde{X}_{x}\right) \cdot\left(\tilde{X}_{x_{1}}+\iota^{*} \tilde{X}_{x_{1}}\right) \\
& =1
\end{aligned}
$$

(iii) Since $B \sim 2 L$ and $\pi^{*}(B)=2 E, \pi^{*}(L) \sim E$. Thus,

$$
X_{x} \cdot L^{2}=\frac{1}{2} \cdot\left(\tilde{X}_{x}+\iota^{*} \tilde{X}_{x}\right) \cdot E^{2}=\tilde{X}_{x} \cdot E^{2}=\left(\tilde{X}_{x} \cdot E\right)^{2}=-1 .
$$

(iv) Since $E=\mathbb{P}\left(N^{\vee}\right)$ where $N$ is the normal bundle of $\Delta_{0}$ in $X \times X$, $-E \mid E=\xi$ is the tautological line bundle on $E$. Since $N=T_{\Delta_{0}}$,

$$
\xi^{2}=-(\pi \mid E)^{*} c_{1}(N) \cdot \xi-c_{2}(N)=(\pi \mid E)^{*} K_{\Delta_{0}} \cdot \xi+\left(K_{X}^{2}-12 \chi\left(\mathcal{O}_{X}\right)\right) .
$$


It follows that $\xi^{3}=\left(2 K_{X}^{2}-12 \chi\left(\mathcal{O}_{X}\right)\right) \cdot \xi$. Therefore,

$$
L^{4}=\frac{1}{2} \cdot E^{4}=-\frac{1}{2} \cdot \xi^{3}=6 \chi\left(\mathcal{O}_{X}\right)-K_{X}^{2} .
$$

(v) Note that $(\alpha \otimes 1) \mid E=(\pi \mid E)^{*} \alpha$ since $\Delta_{0} \cong X$. Thus, $\left[\mathcal{Z}_{2}\right] / \alpha \cdot L^{3}=\frac{1}{2} \cdot(\alpha \otimes 1+1 \otimes \alpha) \cdot E^{3}=(\alpha \otimes 1) \cdot E^{3}=(\pi \mid E)^{*} \alpha \cdot \xi^{2}=\alpha \cdot K_{X}$

(vi) Again since $(\alpha \otimes 1)\left|E=(\pi \mid E)^{*} \alpha=(1 \otimes \alpha)\right| E$, we have

$$
\begin{aligned}
{\left[\mathcal{Z}_{2}\right] / \alpha \cdot\left[\mathcal{Z}_{2}\right] / \beta \cdot L^{2} } & =\frac{1}{2} \cdot(\alpha \otimes 1+1 \otimes \alpha) \cdot(\beta \otimes 1+1 \otimes \beta) \cdot E^{2} \\
& =-2 \cdot(\pi \mid E)^{*} \alpha \cdot(\pi \mid E)^{*} \beta \cdot \xi \\
& =-2(\alpha \cdot \beta) .
\end{aligned}
$$

We continue the calculation of $s_{4}\left(\mathcal{E}_{\zeta}^{2,0}\right)$. By Lemma $5.8(\mathrm{i}),\left(\left[\mathcal{Z}_{2}\right] / \zeta\right)^{4}=$ $3\left(\zeta^{2}\right)^{2}$. It follows from the above Claim with a straightforward computation that

$$
s_{4}\left(\mathcal{E}_{\zeta}^{2,0}\right)=\frac{\left(\zeta^{2}\right)^{2}}{2}-5 \zeta^{2}-\frac{5}{2} \zeta \cdot K_{X}+\left(6 \chi\left(\mathcal{O}_{X}\right)-K_{X}^{2}\right) .
$$

Next, we compute the Chern and Segre classes of $\mathcal{E}_{\zeta}^{0,2}$ on $H_{2}$.

Lemma 6.11. $c_{3}\left(\mathcal{E}_{\zeta}^{0,2}\right)=c_{4}\left(\mathcal{E}_{\zeta}^{0,2}\right)=0, c_{1}\left(\mathcal{E}_{\zeta}^{0,2}\right)=\left[\mathcal{Z}_{2}\right] /\left(\zeta-K_{X}\right)+L$, and

$$
c_{2}\left(\mathcal{E}_{\zeta}^{0,2}\right)=\frac{1}{2}\left[L \cdot\left[\mathcal{Z}_{2}\right] /\left(\zeta-K_{X}\right)+\left[\left[\mathcal{Z}_{2}\right] /\left(\zeta-K_{X}\right)\right]^{2}-\left(\zeta-K_{X}\right)^{2} \cdot X_{x}\right] .
$$

Proof. Let $\ell_{\zeta}=2$ and $k=2$ in Lemma 5.11. By Lemma 6.6 (i),

$$
\left(\operatorname{det} T_{\mathcal{Z}_{2}}\right)^{-1}=\mathcal{O}_{\mathcal{Z}_{2}}\left(K_{\mathcal{Z}_{2}}\right)=\left(\pi_{2} \cdot i\right)^{*} \mathcal{O}_{H_{2}}\left(K_{H_{2}}+L\right) .
$$

Let $N_{\mathcal{Z}_{2}}$ be the normal bundle of $\mathcal{Z}_{2}$ in $X \times H_{2}$. Since $\mathcal{Z}_{2}$ is smooth and has codimension 2 in $X \times H_{2}, E x t^{1}=\operatorname{Ext}^{1}\left(I_{\mathcal{Z}_{2}}, \mathcal{O}_{X \times H_{2}}\right)$ is isomorphic to

$$
\operatorname{det} N_{\mathcal{Z}_{2}}=i^{*} \operatorname{det} T_{X \times H_{2}} \otimes\left(\operatorname{det} T_{\mathcal{Z}_{2}}\right)^{-1}=\mathcal{O}_{\mathcal{Z}_{2}}\left(\left(\pi_{2} \cdot i\right)^{*} L-\left(\pi_{1} \cdot i\right)^{*} K_{X}\right) .
$$

By Lemma 5.11 (i), $\mathcal{E}_{\zeta}^{0,2}$ sits in an exact sequence

$$
0 \rightarrow\left[\mathcal{O}_{H_{2}}\right]^{\oplus h(\zeta)} \rightarrow \mathcal{E}_{\zeta}^{0,2} \rightarrow\left(\pi_{2} \cdot i\right)_{*} \mathcal{O}_{\mathcal{Z}_{2}}\left(\left(\pi_{2} \cdot i\right)^{*} L+\left(\pi_{1} \cdot i\right)^{*}\left(\zeta-K_{X}\right)\right) \rightarrow 0 .
$$


Note that $\left(\pi_{2} \cdot i\right)_{*}\left(\pi_{2} \cdot i\right)^{*} L=2 L$. Thus, by Lemma 6.6 (ii), $c_{1}\left(\mathcal{E}_{\zeta}^{0,2}\right)=\left(\pi_{2} \cdot i\right)_{*}\left[\left(\pi_{2} \cdot i\right)^{*} L+\left(\pi_{1} \cdot i\right)^{*}\left(\zeta-K_{X}\right)\right]-L=\left[\mathcal{Z}_{2}\right] /\left(\zeta-K_{X}\right)+L$.

Also, Lemma 6.6 (ii) together with a straightforward calculation gives

$$
c_{2}\left(\mathcal{E}_{\zeta}^{0,2}\right)=\frac{1}{2}\left[L \cdot\left[\mathcal{Z}_{2}\right] /\left(\zeta-K_{X}\right)+\left[\left[\mathcal{Z}_{2}\right] /\left(\zeta-K_{X}\right)\right]^{2}-\left(\zeta-K_{X}\right)^{2} \cdot X_{x}\right]
$$

where we have used the projection formula

$$
\left(\pi_{2} \cdot i\right)_{*}\left[\left(\pi_{2} \cdot i\right)^{*} L \cdot\left(\pi_{1} \cdot i\right)^{*}\left(\zeta-K_{X}\right)\right]=L \cdot\left(\pi_{2} \cdot i\right)_{*}\left(\pi_{1} \cdot i\right)^{*}\left(\zeta-K_{X}\right)
$$

and the fact that $\left(\pi_{2} \cdot i\right)_{*}\left(\pi_{2} \cdot i\right)^{*} L^{2}=2 L^{2}$.

The following follows from Lemma 6.11 and Remark 5.6.

Corollary 6.12. The Segre classes of $\mathcal{E}_{\zeta}^{0,2}$ are given by

$$
\begin{aligned}
s_{1}\left(\mathcal{E}_{\zeta}^{0,2}\right)= & {\left[\mathcal{Z}_{2}\right] /\left(K_{X}-\zeta\right)-L } \\
s_{2}\left(\mathcal{E}_{\zeta}^{0,2}\right)= & \frac{1}{2}\left[\left[\left[\mathcal{Z}_{2}\right] /\left(\zeta-K_{X}\right)\right]^{2}+\right. \\
& \left.+3\left[\mathcal{Z}_{2}\right] /\left(\zeta-K_{X}\right) \cdot L+2 L^{2}+\left(\zeta-K_{X}\right)^{2} \cdot X_{x}\right] \\
s_{3}\left(\mathcal{E}_{\zeta}^{0,2}\right)= & -\left[\left[\mathcal{Z}_{2}\right] /\left(\zeta-K_{X}\right)\right]^{2} \cdot L-2\left[\mathcal{Z}_{2}\right] /\left(\zeta-K_{X}\right) \cdot L^{2}-L^{3} \\
& \quad-\left(\zeta-K_{X}\right)^{2} \cdot X_{x} \cdot\left[\mathcal{Z}_{2}\right] /\left(\zeta-K_{X}\right)-\left(\zeta-K_{X}\right)^{2} \cdot X_{x} \cdot L \\
s_{4}\left(\mathcal{E}_{\zeta}^{0,2}\right)= & \frac{\left(\left(K_{X}-\zeta\right)^{2}\right)^{2}}{2}-5\left(K_{X}-\zeta\right)^{2}- \\
& -\frac{5}{2}\left(K_{X}-\zeta\right) \cdot K_{X}+\left(6 \chi\left(\mathcal{O}_{X}\right)-K_{X}^{2}\right) .
\end{aligned}
$$

Proof. The calculation of $s_{4}\left(\mathcal{E}_{\zeta}^{0,2}\right)$ is similar to that of $s_{4}\left(\mathcal{E}_{\zeta}^{2,0}\right)$ in Corollary 6.10 .

Note that $s_{4}\left(\mathcal{E}_{\zeta}^{0,2}\right)$ may be obtained from $s_{4}\left(\mathcal{E}_{\zeta}^{2,0}\right)$ by replacing $\zeta$ by $K_{X}-\zeta$, and indeed this holds more generally for $s_{i}$ when we add the sign $(-1)^{i}$.

Now we compute the Chern and Segre classes of $\mathcal{E}_{\zeta}^{1,1}$ on $X \times X$.

Lemma 6.13. Let $\tau_{1}$ and $\tau_{2}$ be the two natural projections of $X \times X$ to $X$, let $\Delta_{0}$ be the diagonal in $X \times X$, and let $j: \Delta_{0} \rightarrow X \times X$ be the inclusion. 
Then

$$
\begin{aligned}
& c_{1}\left(\mathcal{E}_{\zeta}^{1,1}\right)=\tau_{1}^{*} \zeta+\tau_{2}^{*}\left(\zeta-K_{X}\right) \\
& c_{2}\left(\mathcal{E}_{\zeta}^{1,1}\right)=\tau_{1}^{*} \zeta \cdot \tau_{2}^{*}\left(\zeta-K_{X}\right)+\Delta_{0} \\
& c_{3}\left(\mathcal{E}_{\zeta}^{1,1}\right)=\tau_{1}^{*} \zeta \cdot \Delta_{0}-\tau_{2}^{*}\left(\zeta-K_{X}\right) \cdot \Delta_{0}-j_{*} K_{\Delta_{0}} \\
& c_{4}\left(\mathcal{E}_{\zeta}^{1,1}\right)=-\frac{K_{\Delta_{0}}^{2}}{2}
\end{aligned}
$$

Proof. Let $\ell_{\zeta}=2$ and $k=1$ in Lemma 5.11. Recall that $\pi_{1}$ and $\pi_{2}$ are the natural projections of $X \times(X \times X)$ to $X$ and $(X \times X)$ respectively.

Claim 1. $\pi_{2 *}\left(\pi_{1}^{*} \mathcal{O}_{X}(\zeta) \otimes E x t^{1}\right) \cong \tau_{2}^{*} \mathcal{O}_{X}\left(\zeta-K_{X}\right) \otimes I_{\Delta_{0}}$

Proof. Let $\Delta_{12}$ be the diagonal in $X \times X$ which is formed by the first and second factors in $X \times(X \times X)$, and let $\Delta_{13}$ be the diagonal in $X \times X$ which is formed by the first and third factors in $X \times(X \times X)$. Then, $\Delta_{12} \times X$ and $\Delta_{13} \times X$ are smooth codimension 2 subvarieties in $X \times(X \times X)$. Here it is understood that the factor $X$ in $\Delta_{13} \times X$ is embedded as the second factor in $X \times(X \times X)$. Moreover, $\Delta_{12} \times X$ and $\Delta_{13} \times X$ intersect properly along the diagonal $\Delta_{123}$ in $X \times X \times X$. Thus, from Lemma 5.10 (iii), we conclude that

$$
E x t^{1}=E_{x} t^{1}\left(I_{\Delta_{13} \times X}, I_{\Delta_{12} \times X}\right) \cong I \otimes \operatorname{det} N
$$

where $N$ is the normal bundle $\Delta_{13} \times X$ in $X \times(X \times X)$, and $I$ is the ideal sheaf of $\Delta_{123}$ in $\Delta_{13} \times X$. Now, the restriction of $\pi_{2}$ to $\Delta_{13} \times X$ gives an isomorphism from $\Delta_{13} \times X$ to $X \times X$. Via this isomorphism, $\Delta_{123}$ in $\Delta_{13} \times X$ is identified with the diagonal $\Delta_{0}$ in $X \times X$, det $N$ is identified with $\tau_{2}^{*}\left(-K_{X}\right)$, and the restriction $\pi_{1}^{*} \mathcal{O}_{X}(\zeta) \mid\left(\Delta_{13} \times X\right)$ is identified with $\tau_{2}^{*}(\zeta)$. Therefore,

$\pi_{2 *}\left(\pi_{1}^{*} \mathcal{O}_{X}(\zeta) \otimes E x t^{1}\right) \cong \pi_{2 *}\left(\pi_{1}^{*} \mathcal{O}_{X}(\zeta) \otimes I \otimes \operatorname{det} N\right)=\tau_{2}^{*} \mathcal{O}_{X}\left(\zeta-K_{X}\right) \otimes I_{\Delta_{0}}$ 
Note that $\pi_{2 *}\left(\pi_{1}^{*} \mathcal{O}_{X}(\zeta) \otimes \mathcal{O}_{\Delta_{12} \times X}\right)=\tau_{1}^{*}(\zeta)$. Thus by Lemma 5.11 (i) and Claim 1, we have a row exact sequence and a column exact sequence (6.14)

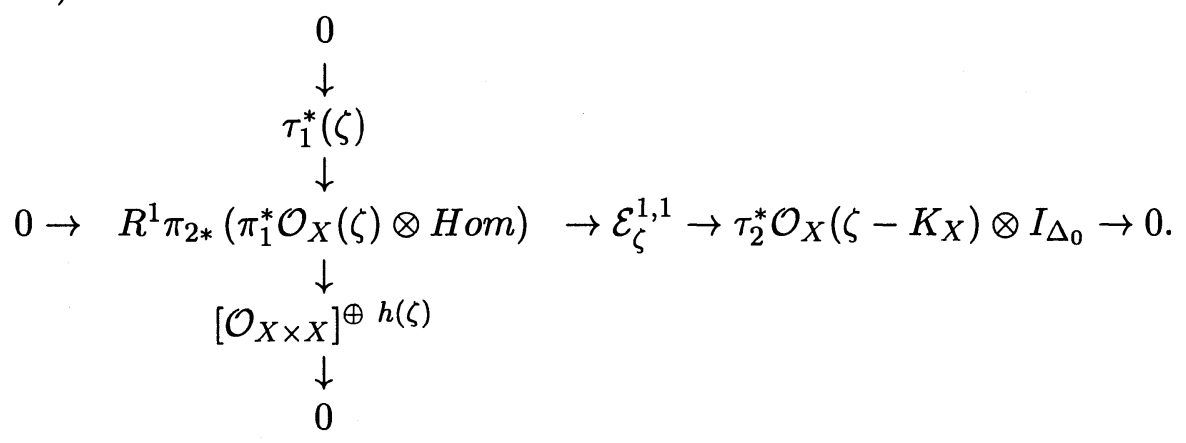

In the next claim, we compute the Chern classes of $I_{\Delta_{0}}$. Clearly, $c_{0}\left(I_{\Delta_{0}}\right)=1$.

Claim 2. $c_{1}\left(I_{\Delta_{0}}\right)=0, c_{2}\left(I_{\Delta_{0}}\right)=\Delta_{0}, c_{3}\left(I_{\Delta_{0}}\right)=-j_{*} K_{\Delta_{0}}, c_{4}\left(I_{\Delta_{0}}\right)=$ $K_{\Delta_{0}}^{2} / 2$.

Proof. Note that $\operatorname{Todd}\left(N_{\Delta_{0}}\right)^{-1}=1+K_{\Delta_{0}} / 2+\left(K_{\Delta_{0}}^{2} / 4-\chi\left(\mathcal{O}_{\Delta_{0}}\right)\right)$. By a formula on p.288 of [12] (a special case of the Grothendieck-Riemann-Roch Theorem),

$$
\begin{aligned}
\operatorname{ch}\left(j ! \mathcal{O}_{\Delta_{0}}\right) & =j_{*}\left(\operatorname{Todd}\left(N_{\Delta_{0}}\right)^{-1} \cdot \operatorname{ch}\left(\mathcal{O}_{\Delta_{0}}\right)\right)=j_{*}\left(\operatorname{Todd}\left(N_{\Delta_{0}}\right)^{-1}\right) \\
& =\Delta_{0}+\frac{j_{*} K_{\Delta_{0}}}{2}+j_{*}\left(\frac{K_{\Delta_{0}}^{2}}{4}-\chi\left(\mathcal{O}_{\Delta_{0}}\right)\right) .
\end{aligned}
$$

Since $\operatorname{ch}\left(j ! \mathcal{O}_{\Delta_{0}}\right)$ is just equal to $\operatorname{ch}\left(j_{*} \mathcal{O}_{\Delta_{0}}\right)$, we obtain $\operatorname{ch}\left(I_{\Delta_{0}}\right)=\operatorname{ch}\left(\mathcal{O}_{X \times X}\right)-\operatorname{ch}\left(j_{*} \mathcal{O}_{\Delta_{0}}\right)=1-\Delta_{0}-\frac{j_{*} K_{\Delta_{0}}}{2}-j_{*}\left(\frac{K_{\Delta_{0}}^{2}}{4}-\chi\left(\mathcal{O}_{\Delta_{0}}\right)\right)$.

From this, the Chern classes of $I_{\Delta_{0}}$ follows immediately. In particular,

$$
c_{4}\left(I_{\Delta_{0}}\right)=\frac{\Delta_{0}^{2}}{2}+j_{*}\left(\frac{3 K_{\Delta_{0}}^{2}}{2}-6 \chi\left(\mathcal{O}_{\Delta_{0}}\right)\right)=\frac{K_{\Delta_{0}}^{2}}{2}
$$


since $\Delta_{0}^{2}=c_{2}\left(T_{X}\right)=12 \chi\left(\mathcal{O}_{X}\right)-K_{X}^{2}$ (see the Example 8.1.12 in [12]).

Now the calculation of the Chern classes of $\mathcal{E}_{\zeta}^{1,1}$ follows from (6.14) and Claim 2. In particular,

$$
\begin{gathered}
c_{4}\left(\mathcal{E}_{\zeta}^{1,1}\right)=-\tau_{1}^{*} \zeta \cdot \tau_{2}^{*}\left(\zeta-K_{X}\right) \cdot \Delta_{0}-\tau_{1}^{*} \zeta \cdot j_{*} K_{\Delta_{0}}+\tau_{2}^{*}\left(\zeta-K_{X}\right)^{2} \cdot \Delta_{0} \\
+2 \tau_{2}^{*}\left(\zeta-K_{X}\right) \cdot j_{*} K_{\Delta_{0}}+\frac{j_{*} K_{\Delta_{0}}^{2}}{2}=-\frac{K_{\Delta_{0}}^{2}}{2}
\end{gathered}
$$

since $\tau_{1}^{*} \zeta \cdot \tau_{2}^{*}\left(\zeta-K_{X}\right) \cdot \Delta_{0}=\zeta \cdot\left(\zeta-K_{X}\right)$ and $\tau_{1}^{*} \zeta \cdot j_{*} K_{\Delta_{0}}=\zeta \cdot K_{X}$.

The next result follows immediately from Lemma 6.13 and Remark 5.6.

Corollary 6.15. Let notations be the same as in Lemma 6.13. Then

$$
\begin{aligned}
s_{1}\left(\mathcal{E}_{\zeta}^{1,1}\right)= & -\tau_{1}^{*} \zeta-\tau_{2}^{*}\left(\zeta-K_{X}\right) \\
s_{2}\left(\mathcal{E}_{\zeta}^{1,1}\right)= & \tau_{1}^{*} \zeta^{2}+\tau_{1}^{*} \zeta \cdot \tau_{2}^{*}\left(\zeta-K_{X}\right)+\tau_{2}^{*}\left(\zeta-K_{X}\right)^{2}-\Delta_{0} \\
s_{3}\left(\mathcal{E}_{\zeta}^{1,1}\right)= & -\tau_{1}^{*} \zeta^{2} \cdot \tau_{2}^{*}\left(\zeta-K_{X}\right)-\tau_{1}^{*} \zeta \cdot \tau_{2}^{*}\left(\zeta-K_{X}\right)^{2} \\
& \quad+\tau_{1}^{*} \zeta \cdot \Delta_{0}+3 \tau_{2}^{*}\left(\zeta-K_{X}\right) \cdot \Delta_{0}+j_{*} K_{\Delta_{0}} \\
s_{4}\left(\mathcal{E}_{\zeta}^{1,1}\right)= & \left(12 \zeta \cdot K_{X}-12 \zeta^{2}-3 K_{X}^{2}\right) .
\end{aligned}
$$

We can now work out (5.7) explicitly for $\ell_{\zeta}=2$ and $j=2,1,0$. For simplicity, let

$$
S_{j}=\sum_{k=0}^{2} S_{j, k}=\sum_{k=0}^{2}\left(\left[\mathcal{Z}_{2-k}\right] / \alpha+\left[\mathcal{Z}_{k}\right] / \alpha\right)^{j} \cdot s_{4-j}\left(\mathcal{E}_{\zeta}^{2-k, k} \oplus\left(\mathcal{E}_{-\zeta}^{k, 2-k}\right)^{\vee}\right)
$$

Lemma 6.17. $S_{2}=64 a^{2}+\left(12 \zeta^{2}+4 K_{X}^{2}-20\right) \alpha^{2}$ where $a=(\zeta \cdot \alpha) / 2$.

Proof. Note that $s_{i}\left(\mathcal{E}_{-\zeta}^{k, 2-k}\right)$ (respectively, $S_{j, k}$ ) can be obtained from $s_{i}\left(\mathcal{E}_{\zeta}^{k, 2-k}\right)$ (respectively, $\left.(-1)^{j} \cdot S_{j, 2-k}\right)$ by replacing $\zeta$ by $-\zeta$. Also, $S_{2,2}$ is equal to

$$
\begin{aligned}
\left(\left[\mathcal{Z}_{2}\right] / \alpha\right)^{2} \cdot s_{2}\left(\mathcal{E}_{\zeta}^{0,2} \oplus\left(\mathcal{E}_{-\zeta}^{2,0}\right)^{\vee}\right)= \\
\quad=\left(\left[\mathcal{Z}_{2}\right] / \alpha\right)^{2} \cdot\left[s_{2}\left(\mathcal{E}_{\zeta}^{0,2}\right)-s_{1}\left(\mathcal{E}_{\zeta}^{0,2}\right) \cdot s_{1}\left(\mathcal{E}_{-\zeta}^{2,0}\right)+s_{2}\left(\mathcal{E}_{-\zeta}^{2,0}\right)\right] .
\end{aligned}
$$


Therefore, by Corollary 6.10 and Corollary 6.12 , we obtain

$$
S_{2,2}+S_{2,0}=32 a^{2}+\left(6 \zeta^{2}+2 K_{X}^{2}-12\right) \alpha^{2}+2\left(\alpha \cdot K_{X}\right)^{2} .
$$

Let $\tau_{1}$ and $\tau_{2}$ be the projections of $X \times X$ to $X$. Then, by Corollary 6.15,

$$
\begin{aligned}
S_{2,1} & =\left(\tau_{1}^{*} \alpha+\tau_{2}^{*} \alpha\right)^{2} \cdot s_{2}\left(\mathcal{E}_{\zeta}^{1,1} \oplus\left(\mathcal{E}_{-\zeta}^{1,1}\right)^{\vee}\right) \\
& =\left(\tau_{1}^{*} \alpha+\tau_{2}^{*} \alpha\right)^{2} \cdot\left[s_{2}\left(\mathcal{E}_{\zeta}^{1,1}\right)-s_{1}\left(\mathcal{E}_{\zeta}^{1,1}\right) \cdot s_{1}\left(\mathcal{E}_{-\zeta}^{1,1}\right)+s_{2}\left(\mathcal{E}_{-\zeta}^{1,1}\right)\right] \\
& =32 a^{2}+\left(6 \zeta^{2}+2 K_{X}^{2}-8\right) \alpha^{2}-2\left(\alpha \cdot K_{X}\right)^{2} .
\end{aligned}
$$

It follows that $S_{2}=\left(S_{2,2}+S_{2,0}\right)+S_{2,1}=64 a^{2}+\left(12 \zeta^{2}+4 K_{X}^{2}-20\right) \alpha^{2}$.

Next, adopting the same method as in the proof of Lemma 6.17, we compute the values of $S_{1}$ and $S_{0}$ in the next two lemmas respectively.

Lemma 6.18. $S_{1}=-\left(48 \zeta^{2}+16 K_{X}^{2}-120\right) a$ where $a=(\zeta \cdot \alpha) / 2$.

Proof. In view of (6.16), we have to compute $S_{1,2}, S_{1,1}$, and $S_{1,0}$. Note that $S_{1,0}$ can be obtained from $-S_{1,2}$ by replacing $\zeta$ by $-\zeta$. Using Corollary 6.10 and Corollary 6.12, we see that $\left(S_{1,2}+S_{1,0}\right)=-\left(24 \zeta^{2}+8 K_{X}^{2}-72\right) a-6(\zeta$. $\left.K_{X}\right)\left(\alpha \cdot K_{X}\right)$. Let $\tau_{1}$ and $\tau_{2}$ be the projections of $X \times X$ to $X$. Then, by Corollary 6.15,

$$
\begin{aligned}
S_{1,1} & =\left(\tau_{1}^{*} \alpha+\tau_{2}^{*} \alpha\right) \cdot s_{3}\left(\mathcal{E}_{\zeta}^{1,1} \oplus\left(\mathcal{E}_{-\zeta}^{1,1}\right)^{\vee}\right) \\
& =-\left(24 \zeta^{2}+8 K_{X}^{2}-48\right) a+6\left(\zeta \cdot K_{X}\right)\left(\alpha \cdot K_{X}\right) .
\end{aligned}
$$

It follows that $S_{1}=\left(S_{1,2}+S_{1,0}\right)+S_{1,1}=-\left(48 \zeta^{2}+16 K_{X}^{2}-120\right) a$.

Lemma 6.19. $S_{0}=18\left(\zeta^{2}\right)^{2}+\left(12 K_{X}^{2}-105\right) \zeta^{2}+\left[2\left(K_{X}^{2}\right)^{2}-50 K_{X}^{2}+96\right]$.

Proof. We need to compute $S_{0,2}, S_{0,1}$, and $S_{0,0}$. Again, $S_{0,0}$ can be obtained from $S_{0,2}$ by replacing $\zeta$ by $-\zeta$. Using Corollary 6.10 and Corollary 6.12, we see that

$$
\left(S_{0,2}+S_{0,0}\right)=9\left(\zeta^{2}\right)^{2}+\left(6 K_{X}^{2}-63\right) \zeta^{2}+\left[\left(K_{X}^{2}\right)^{2}-43 K_{X}^{2}+60\right] .
$$

By Corollary 6.15, $S_{0,1}=9\left(\zeta^{2}\right)^{2}+\left(6 K_{X}^{2}-42\right) \zeta^{2}+\left[\left(K_{X}^{2}\right)^{2}-7 K_{X}^{2}+36\right]$. Therefore,

$$
S_{0}=\left(S_{0,2}+S_{0,0}\right)+S_{0,1}=18\left(\zeta^{2}\right)^{2}+\left(12 K_{X}^{2}-105\right) \zeta^{2}+\left[2\left(K_{X}^{2}\right)^{2}-50 K_{X}^{2}+96\right] .
$$


Now we can calculate the difference $\delta_{w, p}^{X}\left(\mathcal{C}_{-}, \mathcal{C}_{+}\right)$when $\ell_{\zeta}=2$.

Theorem 6.20. Let $\zeta$ define a wall of type $(w, p)$ with $\ell_{\zeta}=2$. Then $\left[\mu_{+}(\alpha)\right]^{d}-\left[\mu_{-}(\alpha)\right]^{d}=(-1)^{h(\zeta)} \cdot\left\{g_{0} \cdot a^{d}+g_{1} \cdot a^{d-2} \cdot \alpha^{2}+g_{2} \cdot a^{d-4} \cdot\left(\alpha^{2}\right)^{2}\right\}$ for $\alpha \in H_{2}(X ; \mathbb{Z})$, where $a$ stands for $(\zeta \cdot \alpha) / 2$ and

$$
\begin{aligned}
& g_{2}=\frac{d !}{2 ! \cdot(d-4) !} \\
& g_{1}=\left(\begin{array}{l}
d \\
2
\end{array}\right) \cdot\left(4 K_{X}^{2}+4 d+8\right) \\
& g_{0}=2 d^{2}+4 d \cdot K_{X}^{2}+2\left(K_{X}^{2}\right)^{2}+13 d+10 K_{X}^{2}+21 .
\end{aligned}
$$

In other words, the difference $\delta_{w, p}^{X}\left(\mathcal{C}_{-}, \mathcal{C}_{+}\right)$is equal to

$$
(-1)^{d} \cdot \varepsilon(\zeta, \Delta) \cdot\left\{g_{0} \cdot\left(\frac{\zeta}{2}\right)^{d}+g_{1} \cdot\left(\frac{\zeta}{2}\right)^{d-2} \cdot q_{X}+g_{2} \cdot\left(\frac{\zeta}{2}\right)^{d-4} \cdot q_{X}^{2}\right\}
$$

Proof. In view of Theorem 5.4 and the notation (6.16), we have

$$
\left[\mu_{+}(\alpha)\right]^{d}-\left[\mu_{-}(\alpha)\right]^{d}=\sum_{j=0}^{4}\left(\begin{array}{l}
d \\
j
\end{array}\right) \cdot(-1)^{h(\zeta)+j} \cdot a^{d-j} \cdot S_{j} .
$$

Now, $S_{4}$ and $S_{3}$ are given by Proposition 5.9 and Proposition 5.12 respectively; $S_{2}, S_{1}$, and $S_{0}$ are computed in the previous three lemmas. So it follows that the coefficient of $(-1)^{h(\zeta)} \cdot a^{d-4} \cdot\left(\alpha^{2}\right)^{2}$ is equal to

$$
g_{2}=\frac{d !}{2 ! \cdot(d-4) !} \text {. }
$$

Similarly, also keeping in mind that $\zeta^{2}=(p+8)=(5-d)$, we have

$$
\begin{aligned}
g_{1}=\left(\begin{array}{l}
d \\
2
\end{array}\right) \cdot\left(12 \zeta^{2}+4 K_{X}^{2}+16 d-52\right)=\left(\begin{array}{l}
d \\
2
\end{array}\right) \cdot\left(4 K_{X}^{2}+4 d+8\right) \\
g_{0}=64 \cdot\left(\begin{array}{l}
d \\
2
\end{array}\right)+\left(48 \zeta^{2}+16 K_{X}^{2}-120\right) \cdot d+ \\
\quad+\left[18\left(\zeta^{2}\right)^{2}+12 \zeta^{2} \cdot K_{X}^{2}+2\left(K_{X}^{2}\right)^{2}-105 \zeta^{2}-50 K_{X}^{2}+96\right] \\
=2 d^{2}+4 d \cdot K_{X}^{2}+2\left(K_{X}^{2}\right)^{2}+13 d+10 K_{X}^{2}+21 .
\end{aligned}
$$


Corollary 6.21. Let $\zeta$ define a wall of type $(w, p)$ with $\ell_{\zeta} \leq 2$. Then, the difference $\delta_{w, p}^{X}\left(\mathcal{C}_{-}, \mathcal{C}_{+}\right)$of Donaldson polynomial invariants is a polynomial in $\zeta$ and $q_{X}$ with coefficients involving only $\zeta^{2}$, homotopy invariants of $X$, and universal constants.

Proof. Follows from Theorems 6.1, 6.4, and 6.20.

Finally, we compute the difference $\left[\mu_{+}(\alpha)\right]^{d-2} \cdot \nu_{+}-\left[\mu_{-}(\alpha)\right]^{d-2} \cdot \nu_{-}$for $\ell_{\zeta}=2$.

Theorem 6.22. Let $\zeta$ define a wall of type $(w, p)$ with $\ell_{\zeta}=2$, and let $d=-p-3$. Then, $\left[\mu_{+}(\alpha)\right]^{d-2} \cdot \nu_{+}-\left[\mu_{-}(\alpha)\right]^{d-2} \cdot \nu_{-}$is equal to

$$
\frac{1}{4} \cdot(-1)^{h(\zeta)+1} \cdot\left\{\tilde{g}_{0} \cdot a^{d-2}+\tilde{g}_{1} \cdot a^{d-4} \cdot \alpha^{2}+\tilde{g}_{2} \cdot a^{d-6} \cdot\left(\alpha^{2}\right)^{2}\right\}
$$

for $\alpha \in H_{2}(X ; \mathbb{Z})$, where $a$ stands for $(\zeta \cdot \alpha) / 2$ and

$$
\begin{aligned}
& \tilde{g}_{2}=\frac{(d-2) !}{2 ! \cdot(d-6) !} \\
& \tilde{g}_{1}=\left(\begin{array}{c}
d-2 \\
2
\end{array}\right) \cdot\left(4 K_{X}^{2}+4 d-40\right) \\
& \tilde{g}_{0}=2 d^{2}+4 d \cdot K_{X}^{2}+2\left(K_{X}^{2}\right)^{2}-35 d-38 K_{X}^{2}-99 .
\end{aligned}
$$

Proof. By Theorem $5.5,\left[\mu_{+}(\alpha)\right]^{d-2} \cdot \nu_{+}-\left[\mu_{-}(\alpha)\right]^{d-2} \cdot \nu_{-}$is equal to $\frac{1}{4} \cdot \sum_{j=0}^{4}\left(\begin{array}{c}d-2 \\ j\end{array}\right) \cdot(-1)^{h(\zeta)+1+j} \cdot a^{d-2-j} \cdot S_{j}-\sum_{j=0}^{2}\left(\begin{array}{c}d-2 \\ j\end{array}\right) \cdot(-1)^{h(\zeta)+1+j} \cdot a^{d-2-j} \cdot T_{j}$ where for simplicity we have defined $T_{j}=\sum_{k=0}^{2} T_{j, k}$ as $\sum_{k=0}^{2}\left(\left[\mathcal{Z}_{2-k}\right] / \alpha+\left[\mathcal{Z}_{k}\right] / \alpha\right)^{j} \cdot\left(\left[\mathcal{Z}_{2-k}\right]+\left[\mathcal{Z}_{k}\right]\right) / x \cdot s_{2-j}\left(\mathcal{E}_{\zeta}^{2-k, k} \oplus\left(\mathcal{E}_{-\zeta}^{k, 2-k}\right)^{\vee}\right)$.

Next, we compute $T_{0}$. Using Corollary 6.10 and Corollary 6.12, we obtain

$$
\begin{aligned}
T_{0,0} & =X_{x} \cdot s_{2}\left(\mathcal{E}_{\zeta}^{2,0} \oplus\left(\mathcal{E}_{-\zeta}^{0,2}\right)^{\vee}\right) \\
& =X_{x} \cdot\left[s_{2}\left(\mathcal{E}_{\zeta}^{2,0}\right)-s_{1}\left(\mathcal{E}_{\zeta}^{2,0}\right) \cdot s_{1}\left(\mathcal{E}_{-\zeta}^{0,2}\right)+s_{2}\left(\mathcal{E}_{-\zeta}^{0,2}\right)\right] \\
& =\left(3 \zeta^{2}+3 \zeta \cdot K_{X}+K_{X}^{2}-3\right) .
\end{aligned}
$$


Note that $T_{0,2}$ can be obtained from $T_{0,0}$ by replacing $\zeta$ by $-\zeta$. Thus,

$$
T_{0,2}=\left(3 \zeta^{2}-3 \zeta \cdot K_{X}+K_{X}^{2}-3\right) .
$$

Similarly, using Corollary 6.15 , we get $T_{0,1}=\left(6 \zeta^{2}+2 K_{X}^{2}-4\right)$. Therefore,

$$
T_{0}=\sum_{k=0}^{2} T_{0, k}=\left(12 \zeta^{2}+4 K_{X}^{2}-10\right) .
$$

By similar but much simpler arguments, we conclude that $T_{1}=-16 a$ and $T_{2}=4 \alpha^{2}$.

From (5.9), (5.12), (6.17), (6.18), and (6.19), we have

$$
\begin{aligned}
& S_{4}=12\left(\alpha^{2}\right)^{2}, \\
& S_{3}=-48 a \cdot \alpha^{2}, \\
& S_{2}=64 a^{2}+\left(12 \zeta^{2}+4 K_{X}^{2}-20\right) \alpha^{2}, \\
& S_{1}=-\left(48 \zeta^{2}+16 K_{X}^{2}-120\right) a, \\
& S_{0}=18\left(\zeta^{2}\right)^{2}+\left(12 K_{X}^{2}-105\right) \zeta^{2}+\left[2\left(K_{X}^{2}\right)^{2}-50 K_{X}^{2}+96\right] .
\end{aligned}
$$

Putting all these together, we see that $\left[\mu_{+}(\alpha)\right]^{d-2} \cdot \nu_{+}-\left[\mu_{-}(\alpha)\right]^{d-2} \cdot \nu_{-}$is equal to

$$
\frac{1}{4} \cdot(-1)^{h(\zeta)+1} \cdot\left\{\tilde{g}_{0} \cdot a^{d-2}+\tilde{g}_{1} \cdot a^{d-4} \cdot \alpha^{2}+\tilde{g}_{2} \cdot a^{d-6} \cdot\left(\alpha^{2}\right)^{2}\right\}
$$

where $\tilde{g}_{0}, \tilde{g}_{1}$, and $\tilde{g}_{2}$ are as defined in the statement of Theorem 6.22 above.

\section{References.}

[1] I.V. Artamkin, Deforming torsion-free sheaves on an algebraic surface, Math. USSR Izv. 36(1991), 449-485.

[2] W. Barth, C. Peters, A. Van de Ven, Compact complex surfaces, Springer, Heidelberg New York, 1984.

[3] I. Dolgachev, Y. Hu, Variation of geometric invariant theory quotients, Preprint.

[4] S.K. Donaldson, Anti-self-dual Yang-Mills connections over complex algebraic surfaces and stable vector bundles, Proc. Lond. Math. Soc. 50(1985), 1-26. 
[5] 26(1987), 141-168.

[6] The orientation of Yang-Mills moduli spaces and 4-manifold topology, J. Differ. Geom. 26(1987), 397-428.

[7] , Polynomial invariants for smooth four-manifolds, Topology 29(1990), 257-315.

[8] G. Ellingsrud, L. Göttsche, Variation of moduli spaces and Donaldson invariants under change of polarization, Preprint.

[9] J. Fogarty, Algebraic families on an algebraic surface, II: The Picard scheme of the punctual Hilbert scheme, Amer. J. Math. 95(1973), 660-687.

[10] R. Friedman, Stable Vector Bundles over Algebraic Varieties, to appear.

[11] , Vector bundles and SO(3)-invariants for elliptic surfaces, Jour. Amer. Math. Soc. 8 (1995), 29-139.

[12] W. Fulton, Intersection Theory, Springer-Verlag, Berlin-Heidelberg-New YorkTokyo, 1984.

[13] D. Gieseker, On the moduli space of vector bundles on an algebraic surface, Ann. Math. 106(1977), 45-60.

[14] L. Göttsche, Change of polarization and Hodge numbers of moduli spaces of torsion free sheaves on surfaces, Preprint.

[15] R. Hartshorne, Algebraic geometry, Springer, Heidelberg New York, 1977.

[16] Y. Hu, W.-P. Li, Variation of the Gieseker and Uhlenbeck compactifications, International J. Math. 6 (1995), 397-418.

[17] D. Kotschick, $S O(3)$-invariants for 4-manifolds with $b_{2}^{+}=1$, Proc. Lond. Math. Soc. 63(1991), 426-448.

[18] D. Kotschick, J.W. Morgan, SO(3)-invariants for 4-manifolds with $b_{2}^{+}=1$. II, J. Differ. Geom. 39(1994), 433-456.

[19] P.B. Kronheimer, T.S. Mrowka, Recurrence relations and asymptotics for fourmanifold invariants, Bull. Amer. Math. Soc. 30(1994), 215-221.

[20] Embedded surfaces and the structure of Donaldson's polynomial invariants, J. Differ. Geom. 3 (1995), 573-734.

[21] J. Li, Algebraic geometric interpretation of Donaldson's polynomial invariants, J. Differ. Geom. 37(1993), 417-466. 
[22] W.-P. Li, Z.B. Qin, Low-degree Donaldson polynomial invariants of rational surfaces, J. Alg. Geom. 2(1993), 413-444.

[23] M. Maruyama, K. Yokogawa, Moduli of parabolic stable sheaves, Math. Ann. 293(1992), 77-99.

[24] K. Matsuki, R. Wentworth, Mumford-Thaddeus principle on the moduli space of vector bundles on a surface, Preprint.

[25] K.C. Mong, On some possible formulation of differential invariants for 4manifolds, J. reine angew. Math. 419(1991), 67-78.

[26] J.W. Morgan, Comparison of the Donaldson polynomial invariants with their algebro geometric analogues, Topology 32(1993), 449-488.

[27] S. Mukai, Symplectic structure of the moduli space of sheaves on an abelian or K3 surface, Invent. Math. 77(1984), 101-116.

[28] On the moduli spaces of bundles on K3 surfaces $I$, in Vector bundles on Algebraic Varieties, Oxford Univ. Press, 1987.

[29] Moduli of vector bundles on K3 surfaces, and symplectic manifolds, Sugaku Exp. 1(1988), 139-174.

[30] Z.B. Qin, Moduli spaces of stable rank-2 bundles on ruled surfaces, Invent. Math. 110(1992), 615-625.

[31] Equivalence classes of polarizations and moduli spaces of sheaves, J. Differ. Geom. 37(1993), 397-415.

[32] C. Simpson, Moduli of representations of the fundamental groups of a smooth projective variety I, II, Preprint.

[33] M. Thaddeus, Stable pairs, linear systems and the Verlinde formula, Invent. Math. 117(1994), 317-353.

[34] Geometric invariant theory and fips, Preprint.

[35] A.N. Tyurin, Algebraic geometric aspects of smooth structures I. Donaldson polynomials, Russ. Math. Serv. 44(1990), 113-178.

[36] C.H. Walter, Irreducibility of moduli spaces of vector bundles on birationally ruled surfaces, Preprint.

[37] H.J. Yang, Transition functions and a blow up formula for Donaldson polynomials, Columbia University Thesis, 1992.

[38] K. Yokogawa, Compactification of moduli of parabolic sheaves and moduli for parabolic Higgs sheaves, J. Math. Kyoto University 33(1993), 451-504. 
Columbia University

NEW YORK, NY 10027, USA

E-MAIL:RF@MATH.COLUMBIA.EDU

AND

OKLahoma State University, STILLWATER, OK 74078, USA

E-MAIL: ZQ@MATH.OKSTATE.EDU

RECEIVEd OCtOBER 20Th, 1994.

Added in the proof. Recently, Ellingsrud and Göttsche obtained detailed information on the transition formula, and showed that there is an algorithm that computes the $S U(2)$ and $S O(3)$ polynomial invariants for the projective plane and other rational surfaces $X$ as long as the corresponding chamber contains an ample divisor $H$ with $H \cdot K_{X}<0$. 